\title{
CURRENT OSTEOMYELITIS MOUSE MODELS, A SYSTEMATIC REVIEW
}

\author{
C. Guarch-Pérez, M. Riool and S.A.J. Zaat*
}

Department of Medical Microbiology and Infection Prevention, Amsterdam UMC, University of Amsterdam, Amsterdam Institute for Infection and Immunity, Amsterdam, the Netherlands

\begin{abstract}
Osteomyelitis is an inflammatory bone disease caused by an infecting microorganism leading to a gradual bone loss. Due to the difficulty in studying osteomyelitis directly in patients, animal models allow researchers to investigate the pathogenesis of the infection and the development of novel prophylactic, anti-inflammatory and antimicrobial treatment strategies. This review is specifically focused on the in vivo mouse osteomyelitis studies available in literature. Thus, a systematic search on Web of Science and PubMed was conducted using the query "(infection) AND (mice OR mouse OR murine) AND (model OR models) AND (arthroplasty OR fracture OR (internal fixator) OR (internal fixation OR prosthesis OR implant OR osteomyelitis)". After critical assessment of the studies according to the inclusion and exclusion criteria, 135 studies were included in the detailed analysis. Based on the model characteristics, the studies were classified into five subject groups: haematogenous osteomyelitis, post-traumatic osteomyelitis, bone-implant-related infection, periprosthetic joint infection, fracture-related infection. In addition, the characteristics of the mice used, such as inbred strain, age or gender, the characteristics of the pathogens used, the inoculation methods, the type of anaesthesia and analgesia used during surgery and the procedures for evaluating the pathogenicity of the infecting micro-organism were described. Overall, the mouse is an excellent first step in vivo model to study the pathogenesis, inflammation and healing process of osteomyelitis and to evaluate novel prophylaxis and treatment strategies.
\end{abstract}

Keywords: Osteomyelitis, in vivo, model, mouse, staphylococci, bone, infection.

*Address for correspondence: Sebastian A.J. Zaat, Department of Medical Microbiology and Infection Prevention, Amsterdam UMC, Meibergdreef 9, 1105 AZ, Amsterdam, the Netherlands.

Telephone number: +31 205664863 Email: s.a.zaat@amsterdamumc.nl

Copyright policy: This article is distributed in accordance with Creative Commons Attribution Licence (http://creativecommons.org/licenses/by-sa/4.0/).

\begin{tabular}{llll}
\hline & List of Abbreviations & FLI & fluorescence imaging \\
& & GM & $\begin{array}{l}\text { genetically modified } \\
\text { immunoglobin } \\
\text { A. baumannii }\end{array}$ \\
AP & Acinetobacter baumannii & Ig & interleukin \\
ATCC & alkaline phosphatase & IL & IL 1 receptor \\
BLI & American type culture collection & IL-1R & immunohistochemistry \\
C. acnes & bioluminescence imaging & IMHC & immunofluorescence \\
C. albicans & Cutibacterium acnes & IMF & in vivo imaging system \\
CCR2 & C-C chemokine receptor type 2 & K. pneumoniae & Klebsiella pneumoniae \\
CFU & colony-forming unit & MCP & monocyte chemoattractant protein \\
Cna & collagen-binding adhesin & MRI & magnetic resonance imaging \\
CRP & C-reactive protein & MRSA & methicillin-resistant S. aureus \\
E. coli & Escherichia coli & NET & neutrophil extracellular trap \\
ELISA & enzyme-linked immunosorbent & P. aeruginosa & Pseudomonas aeruginosa \\
& assay & PET & positron emission tomography \\
FnbA/B & fibronectin-binding proteins A and & PINP & procollagen type I propeptide \\
& B & PJI & peri-prosthetic joint infection \\
FISH & fluorescence in situ hybridisation & PMMA & polymethylmethacrylate
\end{tabular}




$\begin{array}{ll}\text { PMN } & \begin{array}{l}\text { polymorphonuclear leukocytes } \\ \text { peptide nucleic acid fluorescent in } \\ \text { situ hybridisation }\end{array} \\ \text { PSM } & \begin{array}{l}\text { phenol-soluble modulin } \\ \text { real-time quantitative reverse } \\ \text { transcription PCR }\end{array} \\ \text { RNA-Seq } & \text { RNA sequencing } \\ \text { S. agalacticae } & \text { Streptococcus agalacticae } \\ \text { S. aureus } & \text { Staphylococcus aureus } \\ \text { S. epidermidis } & \text { Staphylococcus epidermidis } \\ \text { SACs } & \text { S. aureus abscess communities } \\ \text { SarA } & \text { staphylococcal accessory regulator A } \\ \text { SCV } & \text { small colony variant } \\ \text { SigB } & \text { staphylococcal sigma factor B } \\ \text { SPF } & \text { specified-pathogen free } \\ \text { SEM } & \text { scanning electron microscopy } \\ \text { T1D } & \text { type 1 diabetes } \\ \text { T2D } & \text { type 2 diabetes } \\ \text { TEM } & \text { transmission electron microscopy } \\ \text { Th1 } & \text { T helper 1 cell } \\ \text { Th2 } & \text { T helper 2 cell } \\ \text { TNF } & \text { tumour necrosis factor } \\ \text { TRAP } & \text { tartrate-resistant acid phosphatase } \\ \mu \text { mT } & \text { micro-computed tomography } \\ & \end{array}$

\section{Introduction}

\section{General introduction of osteomyelitis}

Osteomyelitis is an inflammatory bone disease caused by an infecting microorganism leading to a gradual bone loss (Kavanagh et al., 2018). The disease can affect a localised bone section or several parts such as cortex, periosteum and even the surrounding soft tissue. These infections can be caused by a contamination through the bloodstream (known as haematogenous infections), by direct seeding of an open fracture related to trauma or surgery or by a contiguous spread from nearby tissue or a prosthetic device (Kavanagh et al., 2018; Lew and Waldvogel, 2004; Zimmerli and Trampuz, 2011). In fact, the main risk factors associated with osteomyelitis are trauma, orthopaedic devices and diabetic foot infection (Berendt et al., 2008).

\section{Pathophysiology of the disease}

Osteomyelitis begins with the bacterial attachment and subsequent biofilm formation on the bone tissue and/or orthopaedic device. Biofilms consist of a community of bacteria attached to a biological or inert surface that are embedded in a matrix of exopolysaccharide, protein and extracellular DNA (Crabbé et al., 2019). The biofilm structure provides an increased tolerance to antibiotics and immune defences, for instance, due to the presence of bacterial phenotypes with low metabolic activity and SCV (Crabbé et al., 2019).

Osteomyelitis is mainly caused by staphylococci, especially by $S$. aureus and S. epidermidis, but can also be caused by other Gram-positive pathogens such as streptococci, by Gram-negative pathogens such as $P$. aeruginosa and even by a mixture of pathogens (Bernthal et al., 2010; Horst et al., 2012; Inzana et al., 2015b). Furthermore, bacterial infections can find other ways to persist despite treatment (Kavanagh et al., 2018). For example, staphylococci can survive intracellularly in human cells such as macrophages, osteoblasts or even osteocytes causing a chronic persistent osteomyelitis extremely difficult to treat (Boelens et al., 2000; Ellington et al., 2006; Valour et al., 2015; Yang et al., 2018). S. aureus can also form SACs, small but highly persistent microcolonies in the bone and soft tissue (Guggenberger et al., 2012; Hofstee et al., 2020; Tuchscherr et al., 2017), and can hide in canaliculi within the bone, out of reach of phagocytic cells (de Mesy Bentley et al., 2017). In addition, the worldwide increase in antibiotic resistance leaves fewer treatment options available $\left(\mathrm{O}^{\prime}\right.$ Neill, 2014). Consequently, patients require prolonged antibiotic treatment and longer hospitalisation and re-operation, resulting in longer disability and in a dramatic clinical and economic burden for the society. Therefore, it is crucial to develop new prevention, diagnosis and treatment strategies for osteomyelitis (Masters et al., 2019; Moriarty et al., 2016).

Clinical translation and the need for animal models Novel strategies for prevention, diagnosis and treatment should be properly evaluated to ensure their effectiveness before use in patients. However, the diverse incidence rate of osteomyelitis ranging from 1 to $30 \%$, depending on the clinical situation and presence of a device (Metsemakers et al., 2015), the diversity in the anatomical locations affected and the wide range of patients age make it difficult to study the disease within the human population (Lazzarini et al., 2006). Moreover, osteomyelitis can be caused by a broad variety of microorganisms, which may necessitate specific treatment for each pathogen (Arciola et al., 2005b).

To overcome these obstacles, animal models have been developed to study the pathology and pathogenesis of osteomyelitis and the efficacy of prophylactic and treatment regimes. These animal

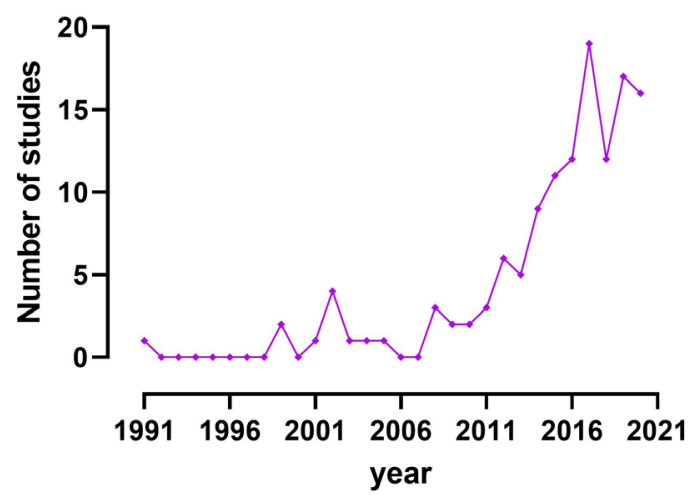

Fig. 1. Number of studies using mouse osteomyelitis models per year between 1991 and 2020. The number of mouse osteomyelitis models steadily increased from 2010 to 2020. 
models should meet specific features to most accurately model the clinical situation in patients, such as a similar bone anatomy, gender influence or susceptibility to infection (Wancket, 2015). Therefore, only research conducted with well-established animal models will contribute to a better understanding of the pathogenesis of the disease and will advance the progress of novel preventive and treatment strategies towards the clinic (Coenye et al., 2018). Although different animal species such as sheep, rabbit, dog and rats have been used for this purpose, the use of mouse models to study osteomyelitis has rapidly increased during the last decade (Fig. 1; Reizner et al., 2014).

\section{Mouse models: advantages and limitations}

Mouse models for osteomyelitis gained popularity particularly with the unravelling of the mouse genome by the Mouse Genome Sequencing Consortium in 2002 (Mouse Genome Sequencing Consortium et al., 2002). The general advantages of mice as models for osteomyelitis studies are their reproducibility (i.d. development of the infection within the mouse strain and availability of the mouse strains to reproduce and continue an experimental approach), their bone physiological and structural similarity to humans, the diversity of genetic and molecular tools available to study them and their relatively low costs. This enables extensive experimental designs, tailored to solve a wide variety of research questions. Furthermore, the mouse has a relatively short life cycle, providing the advantage of a rapid development of the pathological features of osteomyelitis.

Mouse bone is similar in its physiology and structure to human bone, with the presence of trabecular and cortical bone and with similar cell types. However, mice lack a haversian system (osteon) but instead use resorption cavities for bone remodelling that have a similar function to the haversian system (Holstein et al., 2009). This results in a similar physiological control of bone remodelling (Holstein et al., 2009). Compared to larger animals, mice have much smaller bones, making surgical manipulation more challenging (Muschler et al., 2010). This limitation has been successfully solved with high level technology for small rodents that allows the establishment of highly standardised bone surgical procedures. Even the techniques for the insertion of devices such as intramedullary nails or the placement of fixation plates on the bone have been properly standardised, allowing a closer translation to the clinical situation in human patients (HaffnerLuntzer et al., 2016; Histing et al., 2011).

Mice and humans share more than $90 \%$ genetic homology (Mouse Genome Sequencing Consortium et al., 2002). A recent study has demonstrated that gene expression responses of mice and humans to trauma, burns and endotoxemia are significantly correlated

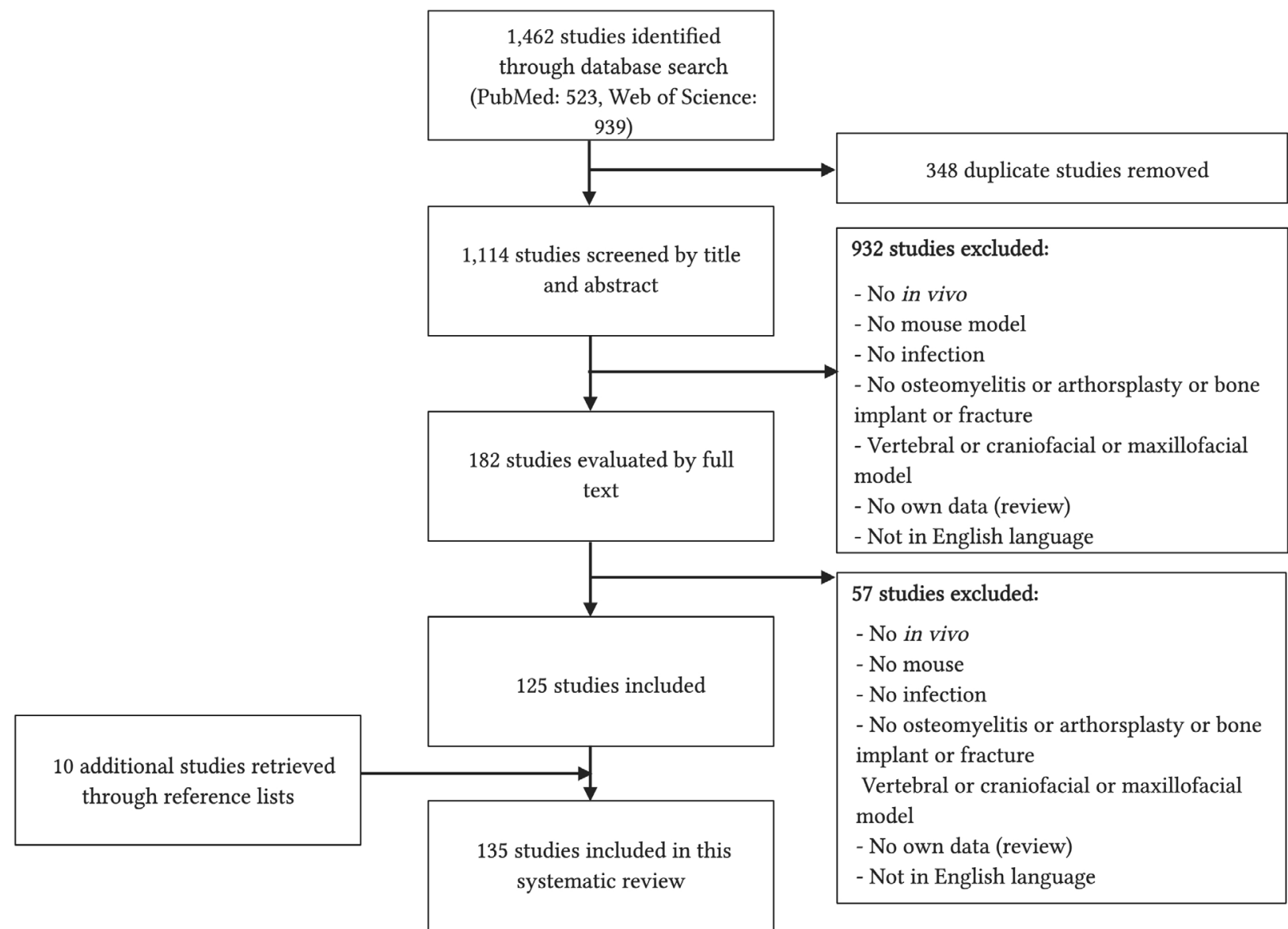

Fig. 2. Schematic flow chart of the steps followed to obtain the collection of studies included in the present systematic review. 
(Takao and Miyakawa, 2015). The immune response to osteomyelitis also shows resemblance between mice and humans. For example, pro-inflammatory cytokines involved in the immune response against bacterial infection such as TNF- $\alpha$, IL-6, IL-1 $\beta$ and IL$17 \mathrm{~A}$ are upregulated in mouse as well as in human patients in the presence of osteomyelitis (Lüthje et al., 2020; Yoshii et al., 2002b). There are also differences. For example, the chemokine CXCL8, which has a central role in the early defence against infection in humans, has not been identified in mice (Lüthje et al., 2020). On the other hand, anti-inflammatory cytokines such as IL-4 are upregulated in both mice and humans during bone repair after infection (Lüthje et al., 2020). Despite the differences, if the detailed course of the immune responses in mice is known, it is possible to compare the results to the human situation.

Regarding osteomyelitis and pathogenesis, numerous studies have successfully established an infection in the bone, bone fractures and bone with an implant (Bernthal et al., 2010; Horst et al., 2012; Inzana et al., 2015b). The present review discusses the major models described in the literature and their most important characteristics.

\section{Aim of the review}

The aim of the present systematic review is to discuss the different types of mouse osteomyelitis models available, to study the pathophysiology and immune response of the disease and the evaluation of the efficacy of novel preventive and treatment strategies. Based on the model characteristics, the published papers were classified into five subject groups: haematogenous osteomyelitis, post-traumatic osteomyelitis, bone-implant-related infection, PJI and fracture-related infection. In addition, the characteristics of the mice used, such as inbred strain, age or gender, the characteristics of the pathogens used, the inoculation methods, the type of anaesthesia and analgesia used during surgery and the procedures for evaluating the infection are described.

\section{Materials and Methods}

\section{Search strategy}

Two databases, Web of Science (Web ref. 1) and PubMed (Web ref. 2), were used to systematically identify studies exploring osteomyelitis in mouse models. The two databases were strategically searched using the search strategy shown in Fig. 2. The search query used for PubMed was (infection[MESH] OR infection[TIAB]) AND (mice[MESH] OR mice[TIAB] OR mouse[TIAB] OR murine[TIAB]) AND (model[TIAB] OR models[TIAB]) AND (arthroplasty[MESH] OR arthroplasty[TIAB] OR fracture[MESH] OR fracture[TIAB] OR (internal fixator)[MESH] OR (internal fixator)[TIAB] OR (internal fixation)[TIAB] OR prosthesis[MESH]
OR prosthesis[TIAB] OR implant[TIAB] OR osteomyelitis[MESH] OR osteomyelitis[TIAB]). A simpler query was used for Web of Science: (infection) AND (mice OR mouse OR murine) AND (model OR models) AND (arthroplasty OR fracture OR (internal fixator) OR (internal fixation OR prosthesis OR implant OR osteomyelitis). The final search was conducted on the 15th of March 2021. In addition, other relevant studies found through the reference lists that met the eligibility criteria were included (Fig. 2).

\section{Inclusion and exclusion criteria}

The inclusion criteria were: (1) in vivo mouse experimental study, (2) bacterial or fungal osteomyelitis studies, (3) long bones. The exclusion criteria were: (1) no osteomyelitis, (2) no bone infection vertebral osteomyelitis, (3) craniofacial or maxillofacial osteomyelitis, (4) non-infection osteomyelitis, (5) no mouse model, (6) no in vivo study, (7) no full-text literature, (8) not written in English language.

\section{Summary of literature search}

Fig. 2 shows the systematic search procedure. A total of 1,462 articles were identified (523 from PubMed and 939 from Web of Science). From those, 348 duplicates were removed. 932 articles were excluded during the initial screening of titles and abstract and 57 during the secondary screening by full reading. 10 additional articles that met the eligibility criteria were retrieved from the reference lists of the papers collected. At the end, 135 studies were included in the present systematic review.

\section{Model design characteristics}

All the studies discovered following the systematic search have in common that they use the mouse as a model to evaluate different aspects of osteomyelitis infection including pathogenesis, prophylaxis and treatment strategies. S. aureus was the main bacterial species used in the experimental studies but also other species, such as A. baumannii and E. coli, were found and are described in the review. A section discussing the main model design characteristics in detail, such as mouse strain, bacterial species, inoculum methods, surgical procedures and evaluation methods, was also included.

The experimental studies were organised into five different categories, each with corresponding tables listing the surgical procedure and bacterial inoculation method used. These categories were haematogenous osteomyelitis, post-traumatic osteomyelitis, bone-implant-related infection, PJI and fracture-related infection (Table 1-6). Each table provides the major details of the published procedure: (1) reference; (2) title; (3) microbiological status of the mice (e.g. SPF), gender and strain; (4) age; (5) time points; (6) bacterial strain, inoculum size and volume; (7) inoculation method; (8) evaluation methods. In Table 2, the size and location of the 


\begin{tabular}{|c|c|c|c|c|c|c|c|c|c|c|c|c|c|c|c|}
\hline 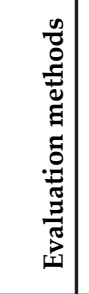 & 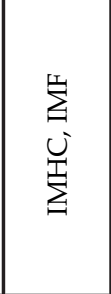 & 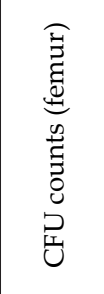 & 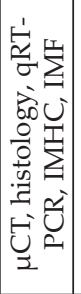 & 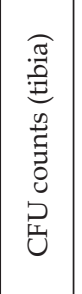 & 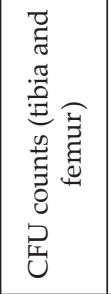 & 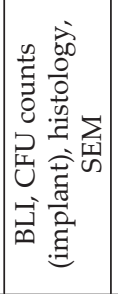 & 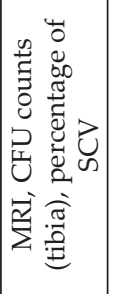 & 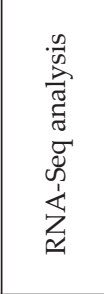 & 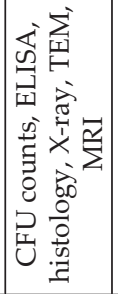 & 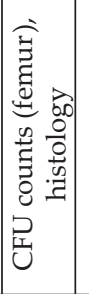 & 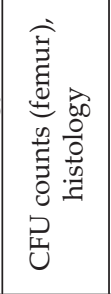 & 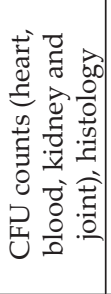 & 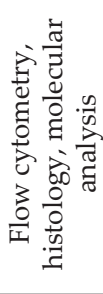 & 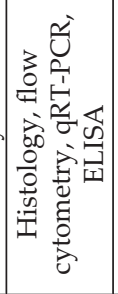 & 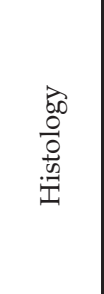 \\
\hline 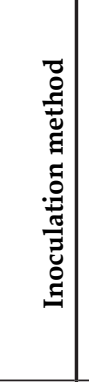 & 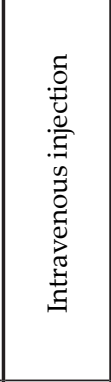 & 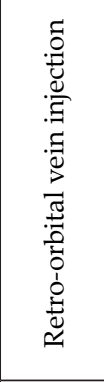 & 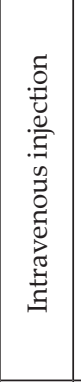 & 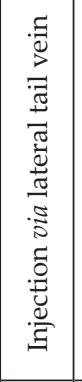 & 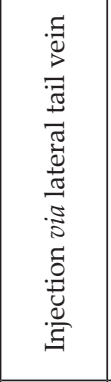 & 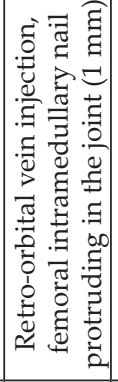 & 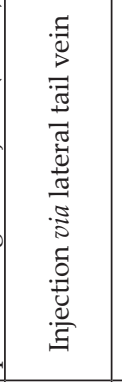 & 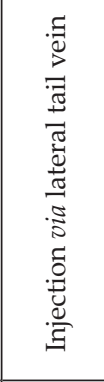 & 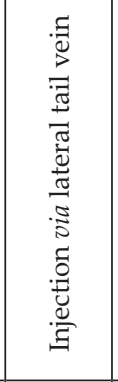 & 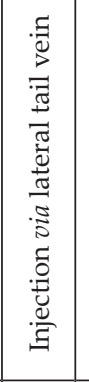 & 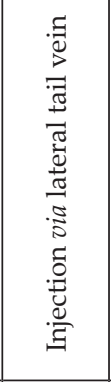 & 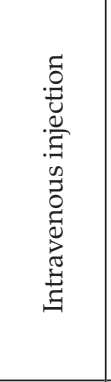 & 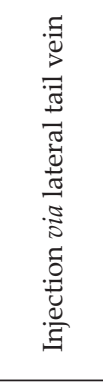 & 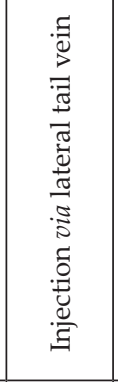 & 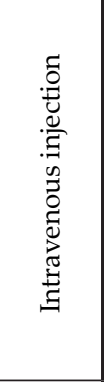 \\
\hline 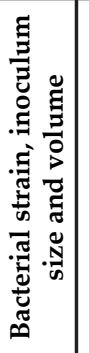 & 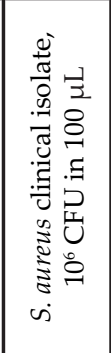 & 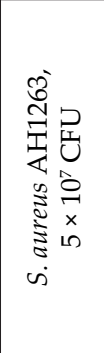 & 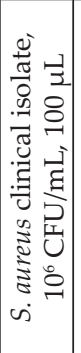 & 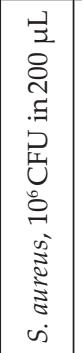 & 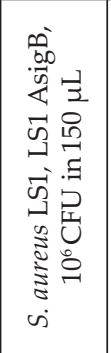 & 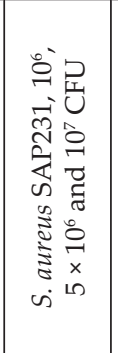 & 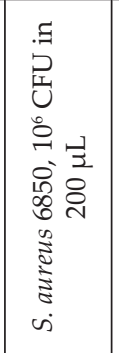 & 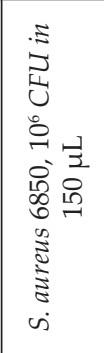 & 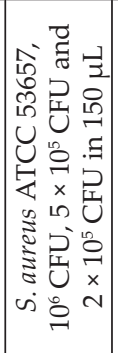 & 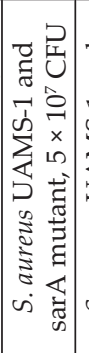 & 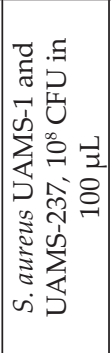 & 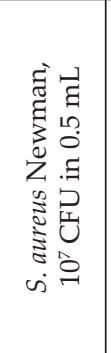 & 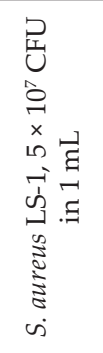 & 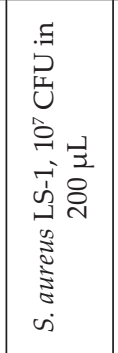 & 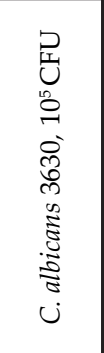 \\
\hline 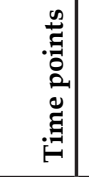 & $\underset{\forall}{\nexists}$ & 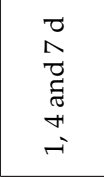 & 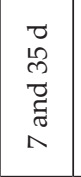 & 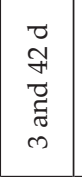 & ' & 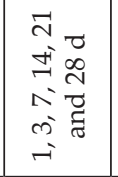 & 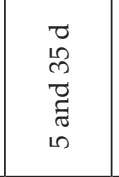 & 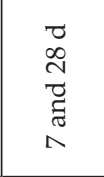 & 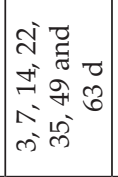 & 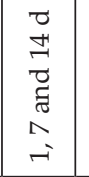 & $\begin{array}{l}\vec{J} \\
\mathbb{J}\end{array}$ & 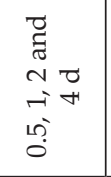 & E & 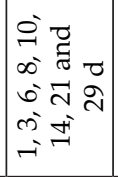 & $\begin{array}{l}\mathbb{D} \\
\stackrel{\infty}{-}\end{array}$ \\
\hline$\stackrel{8}{\alpha}$ & 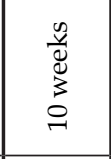 & $\begin{array}{l}\frac{n}{\square} \\
\vdots \\
\Sigma \\
\wedge\end{array}$ & & 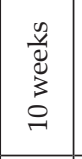 & 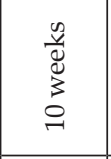 & 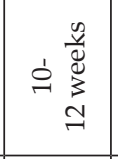 & 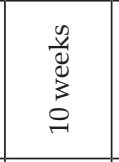 & 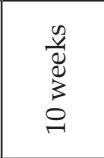 & 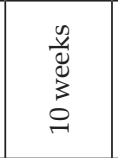 & 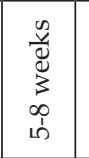 & 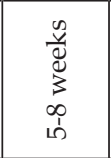 & $\begin{array}{l}\frac{n}{0} \\
0 \\
3 \\
0 \\
0 \\
\infty\end{array}$ & 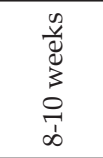 & 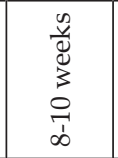 & $\begin{array}{l}\frac{v}{v} \\
\frac{\tilde{v}}{\pi} \\
3 \\
\infty\end{array}$ \\
\hline 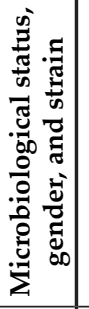 & 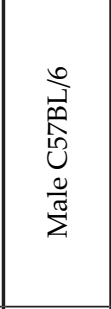 & 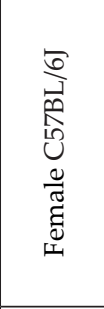 & 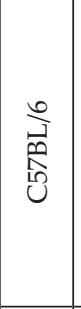 & 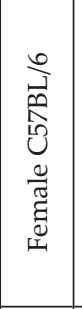 & 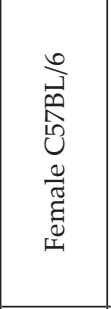 & 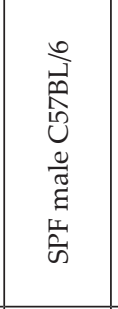 & 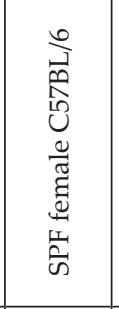 & 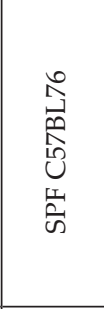 & 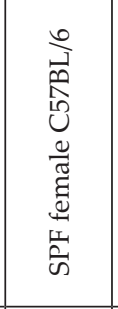 & 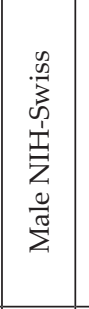 & 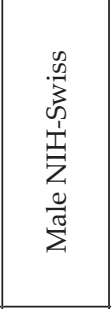 & 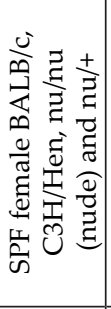 & 茎 & 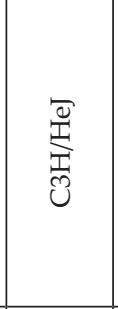 & 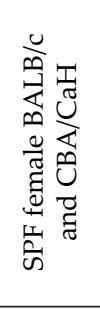 \\
\hline$\stackrel{3}{3}$ & 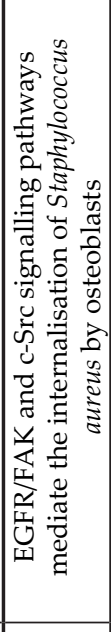 & 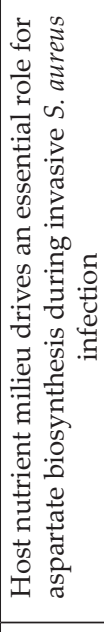 & 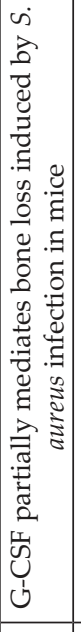 & 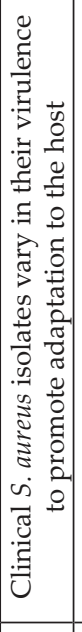 & 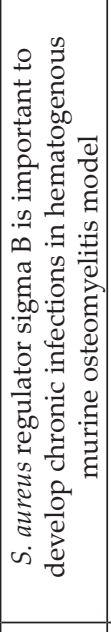 & 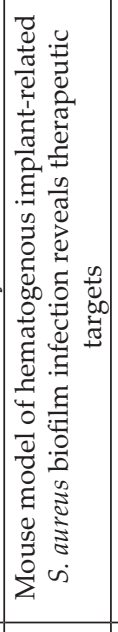 & 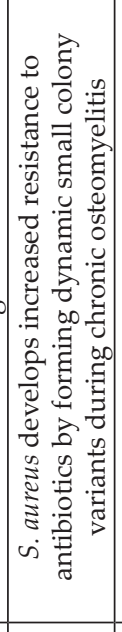 & 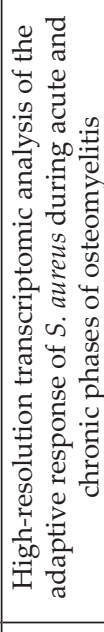 & 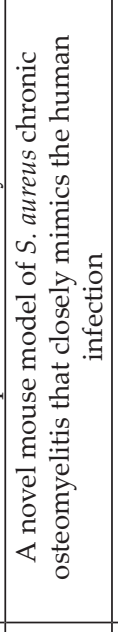 & 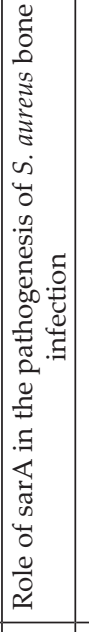 & 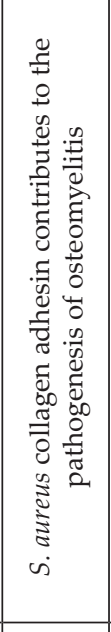 & 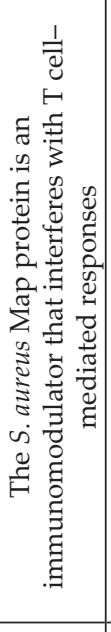 & 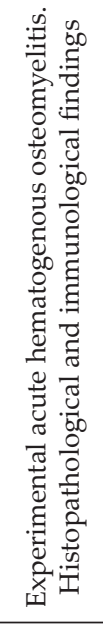 & 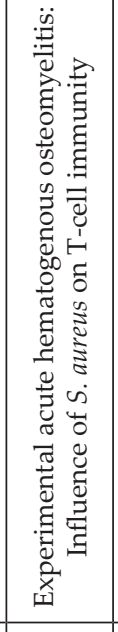 & 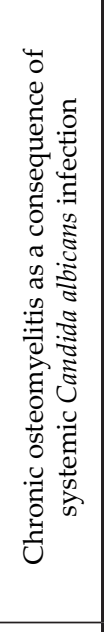 \\
\hline 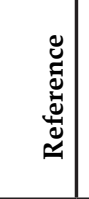 & 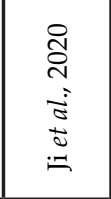 & 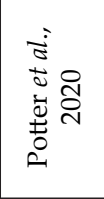 & 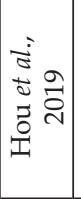 & 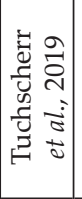 & 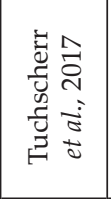 & 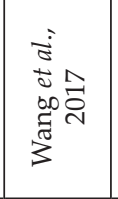 & 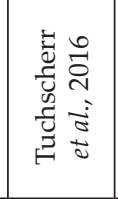 & 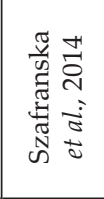 & 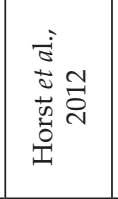 & 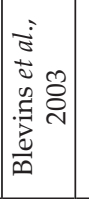 & 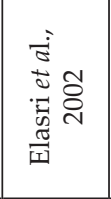 & 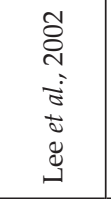 & 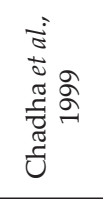 & 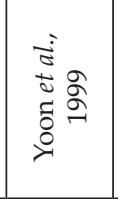 & 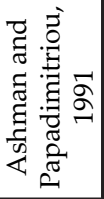 \\
\hline
\end{tabular}




\begin{tabular}{|c|c|c|c|c|c|c|c|c|c|}
\hline 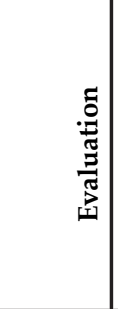 & 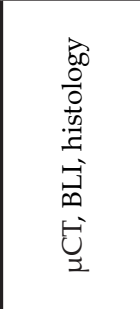 & 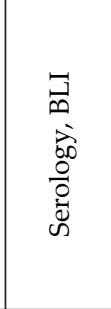 & 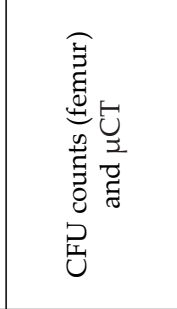 & 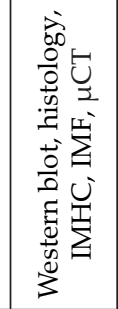 & 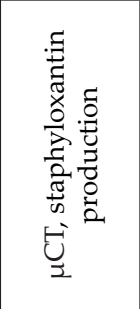 & 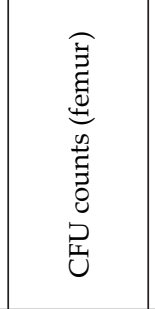 & 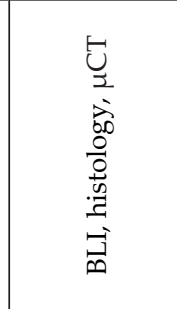 & 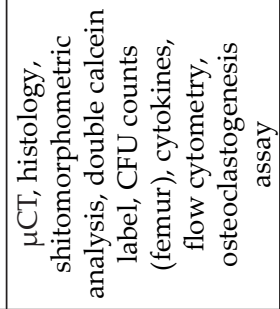 & 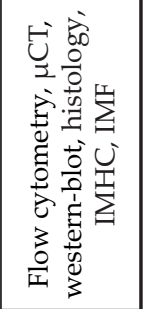 \\
\hline 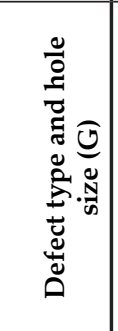 & 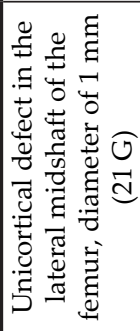 & 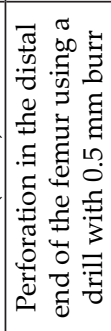 & 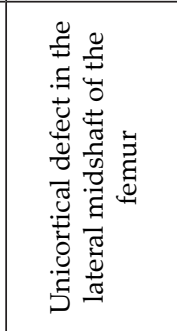 & 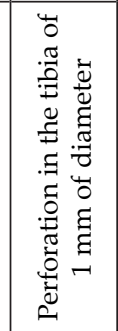 & 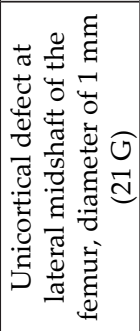 & 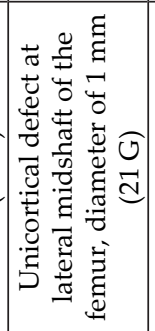 & 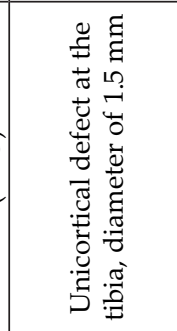 & 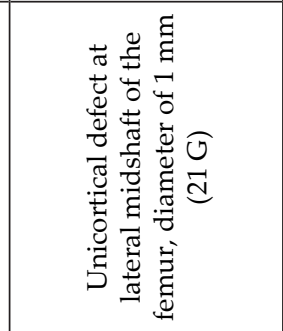 & 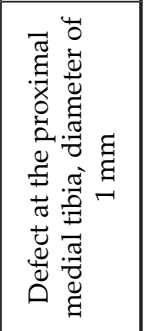 \\
\hline 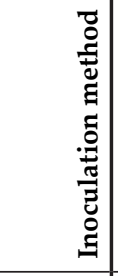 & 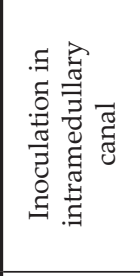 & 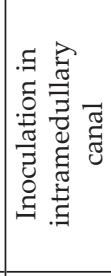 & 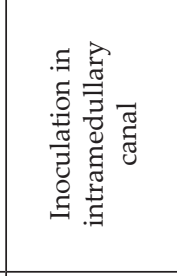 & 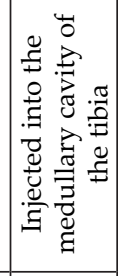 & 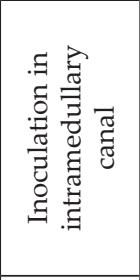 & 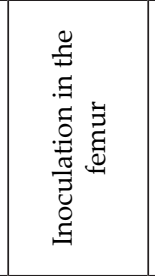 & 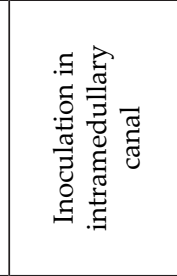 & 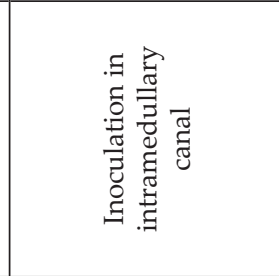 & 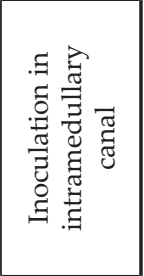 \\
\hline 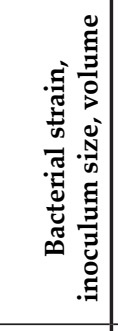 & 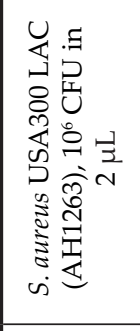 & 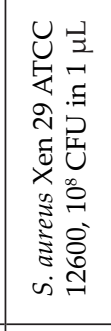 & 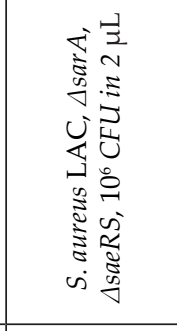 & 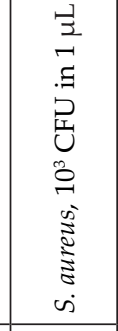 & 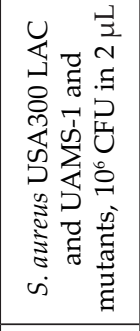 & 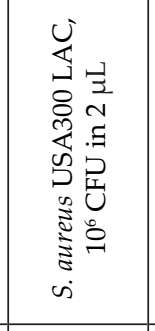 & 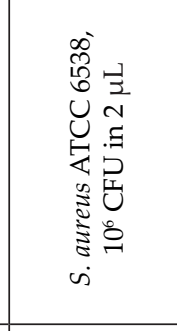 & 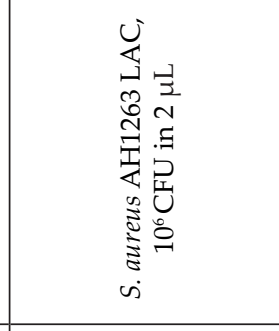 & 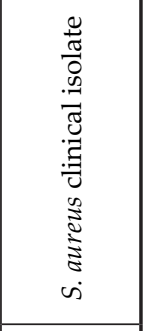 \\
\hline 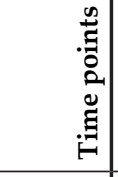 & $\begin{array}{l}\vec{J} \\
\vec{J}\end{array}$ & $\bar{r}$ & $\begin{array}{l}\vec{J} \\
\vec{H}\end{array}$ & $\begin{array}{l}\vec{J} \\
\vec{N} \\
\vec{\Xi} \\
\tilde{\sigma} \\
\triangleq\end{array}$ & $\begin{array}{l}\vec{J} \\
\dot{H}\end{array}$ & 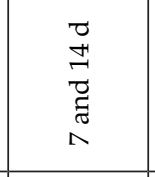 & 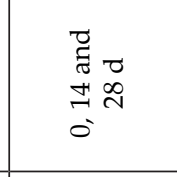 & 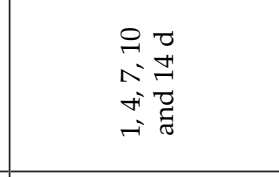 & 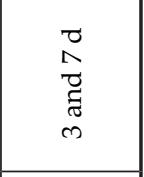 \\
\hline$\stackrel{8}{8}$ & 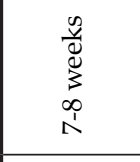 & 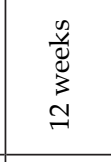 & 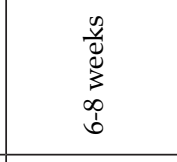 & 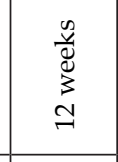 & 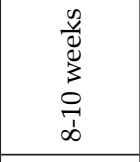 & 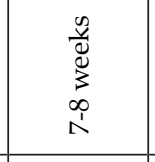 & $\begin{array}{l}n \\
\stackrel{n}{0} \\
\vdots \\
3 \\
\forall\end{array}$ & 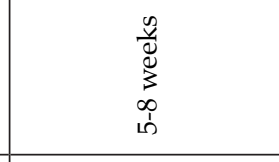 & 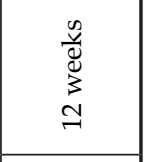 \\
\hline 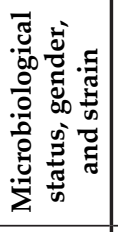 & 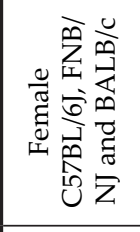 & 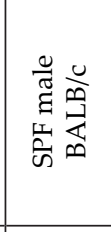 & 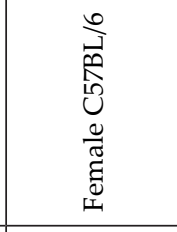 & 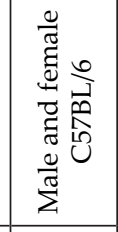 & $\begin{array}{l}\stackrel{0}{\overrightarrow{1}} \\
\stackrel{0}{0.0} \\
\text { b }\end{array}$ & 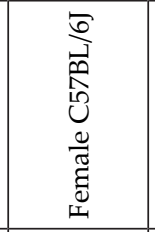 & 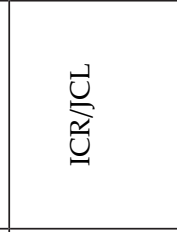 & 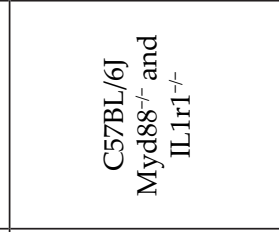 & 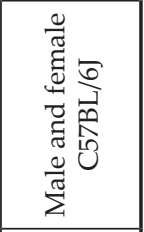 \\
\hline 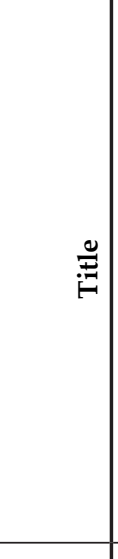 & 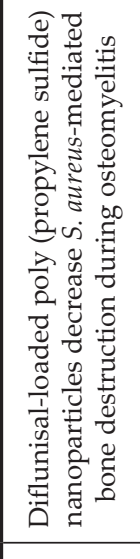 & 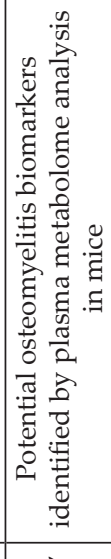 & 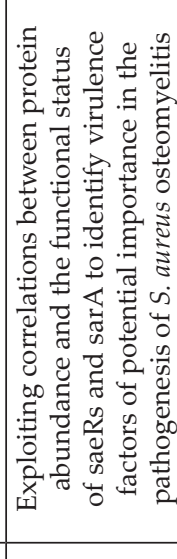 & 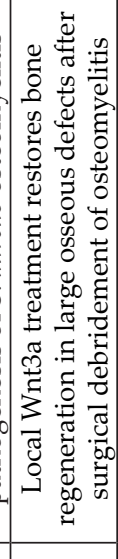 & 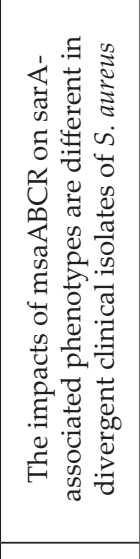 & 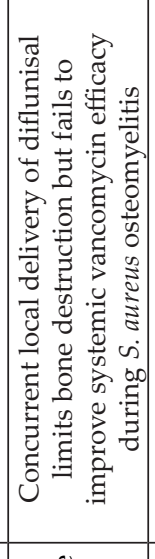 & 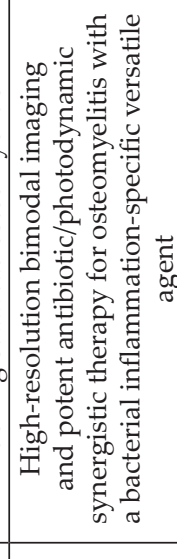 & 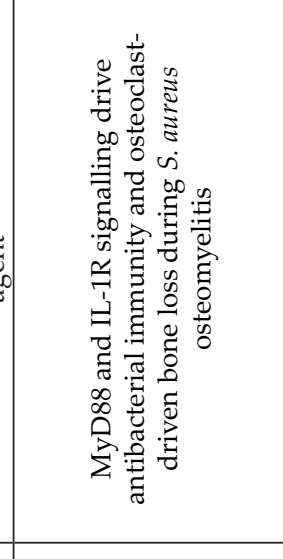 & 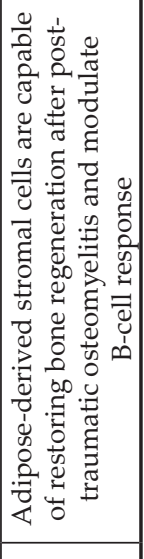 \\
\hline 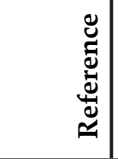 & 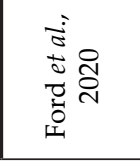 & 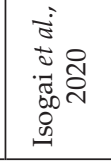 & 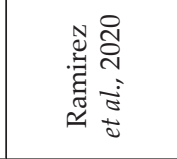 & 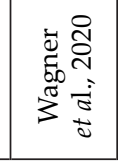 & 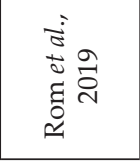 & 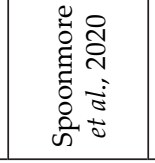 & 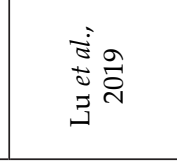 & 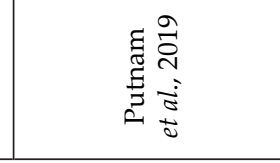 & 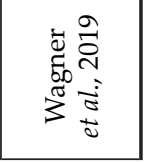 \\
\hline
\end{tabular}




\begin{tabular}{|c|c|c|c|c|c|c|c|c|c|c|}
\hline 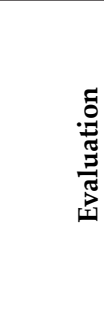 & 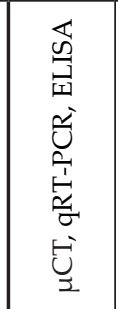 & 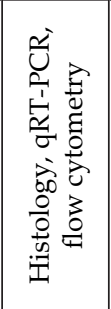 & 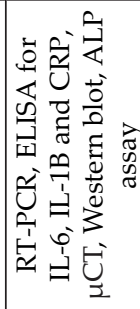 & 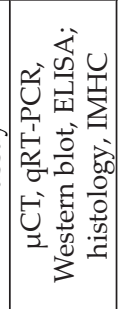 & 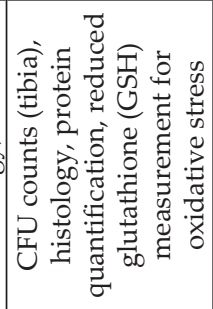 & 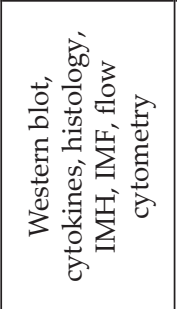 & 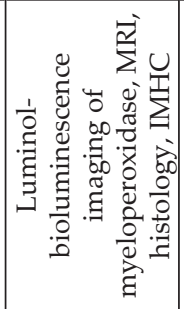 & 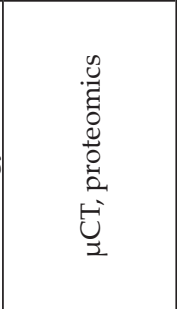 & 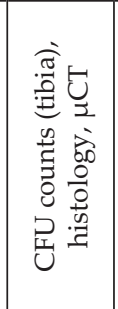 & 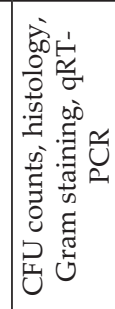 \\
\hline 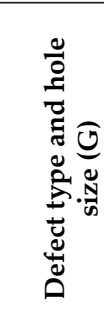 & 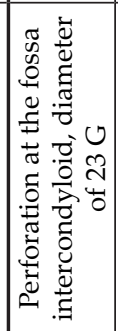 & 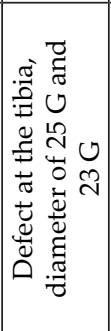 & 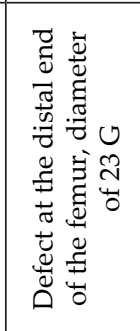 & 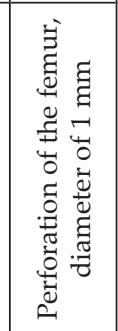 & 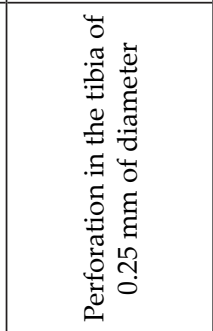 & 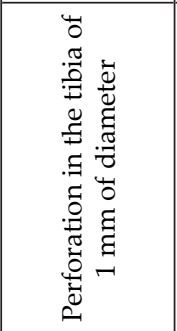 & 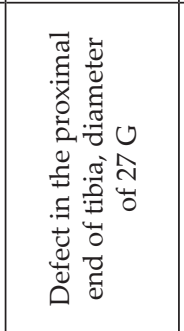 & 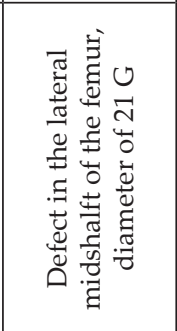 & 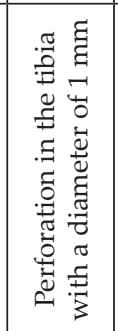 & 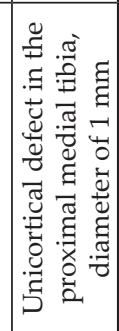 \\
\hline 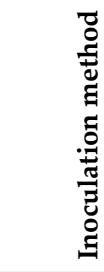 & 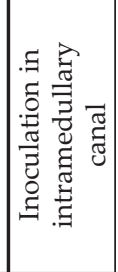 & 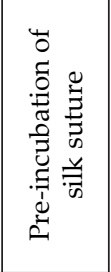 & 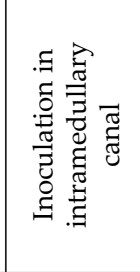 & 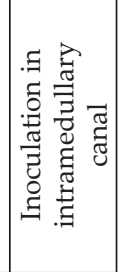 & 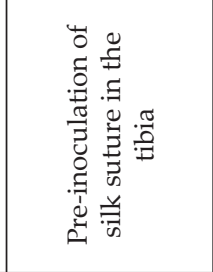 & 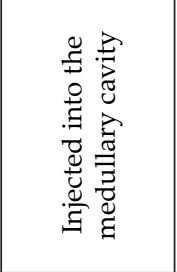 & 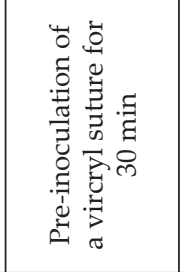 & 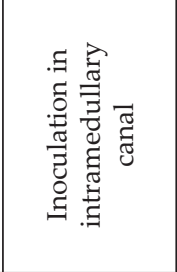 & 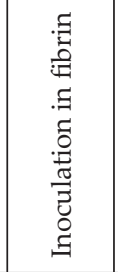 & 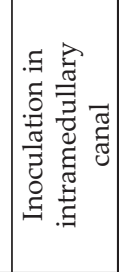 \\
\hline 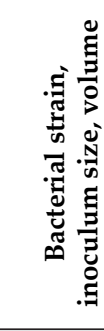 & 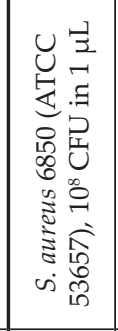 & 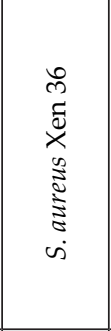 & 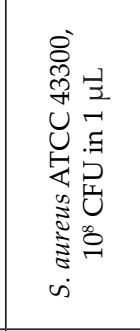 & 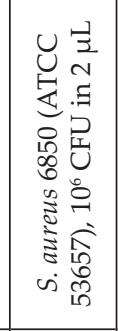 & 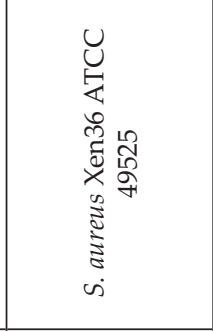 & 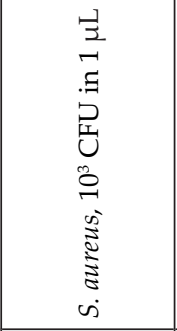 & 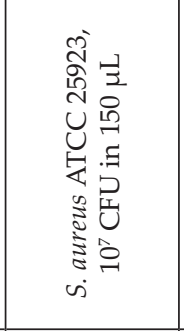 & 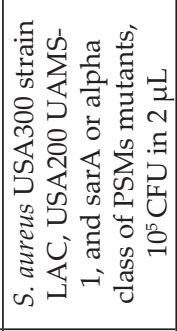 & 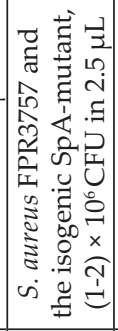 & 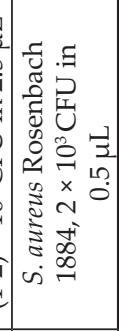 \\
\hline 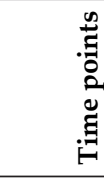 & $\begin{array}{l}\tilde{J} \\
\tilde{J} \\
\tilde{\sigma} \\
\tilde{D}\end{array}$ & & $\begin{array}{l}\tilde{D} \\
\hat{N} \\
\tilde{E} \\
\tilde{N} \\
0 \\
-\end{array}$ & $\underset{J}{J}$ & $\stackrel{\nabla}{\Omega}$ & 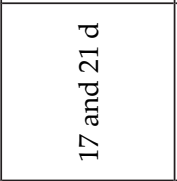 & $\begin{array}{l}\overrightarrow{0} \\
\stackrel{N}{N}\end{array}$ & $\begin{array}{l}\tilde{D} \\
\dot{H}\end{array}$ & $\underset{J}{J}$ & 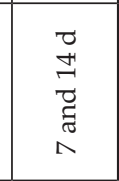 \\
\hline$\stackrel{\circ}{4}$ & $\begin{array}{l}\frac{n}{v} \\
\mathbb{u} \\
3 \\
b\end{array}$ & $\begin{array}{l}\frac{v}{0} \\
\frac{0}{0} \\
\vdots \\
0 \\
0 \\
\infty\end{array}$ & 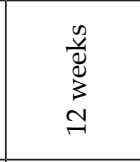 & 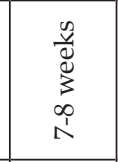 & 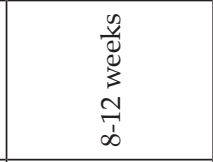 & 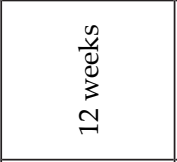 & $\begin{array}{l}0 \\
\frac{n}{0} \\
0 \\
3 \\
0 \\
0 \\
0\end{array}$ & $\begin{array}{l}0,0 \\
\frac{v}{0} \\
\vdots \\
3 \\
0 \\
0 \\
0\end{array}$ & 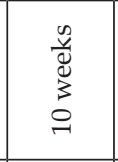 & \\
\hline 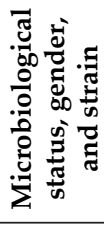 & 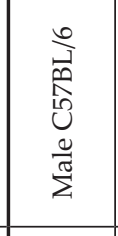 & 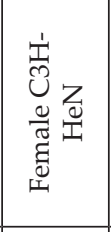 & 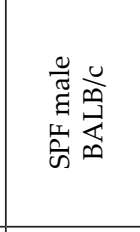 & $\begin{array}{l}0 \\
\stackrel{0}{7} \\
0 \\
0 \\
0 \\
0\end{array}$ & 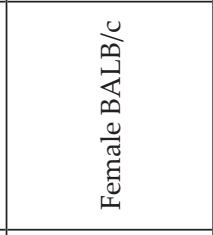 & 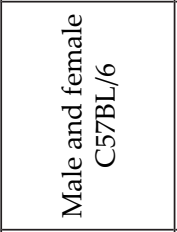 & 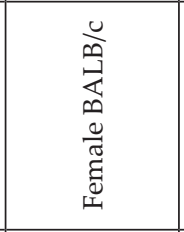 & 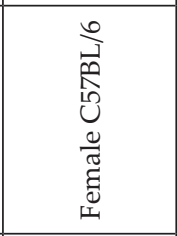 & 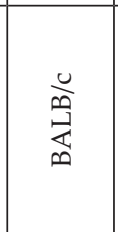 & 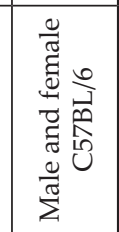 \\
\hline$\stackrel{\Xi}{Z}$ & 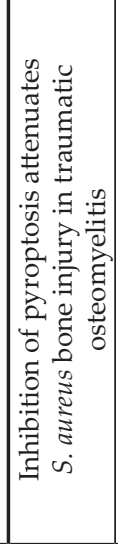 & 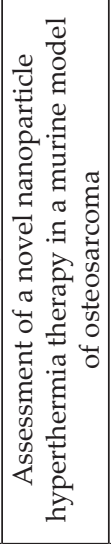 & 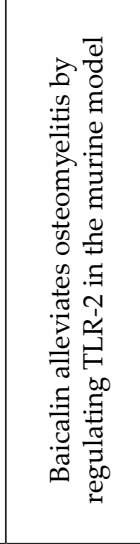 & 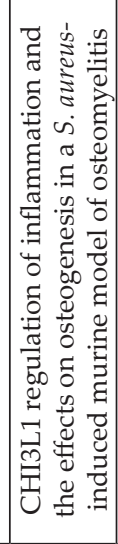 & 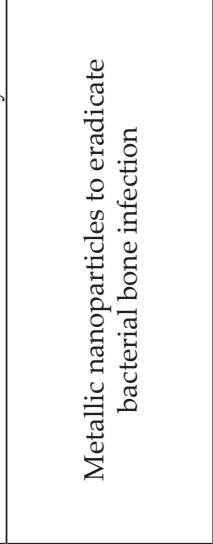 & 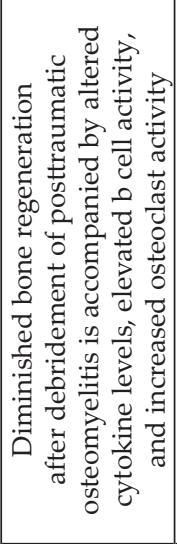 & 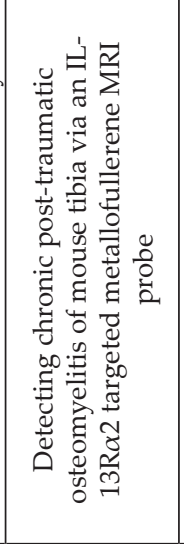 & 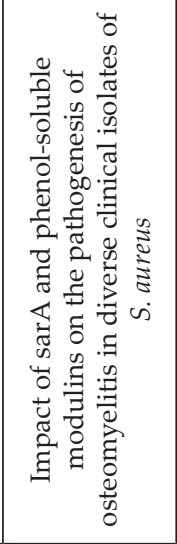 & 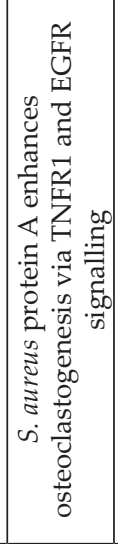 & 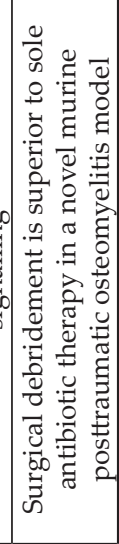 \\
\hline 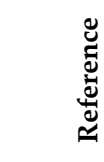 & 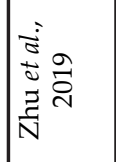 & 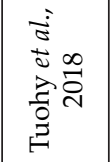 & 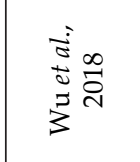 & 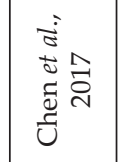 & 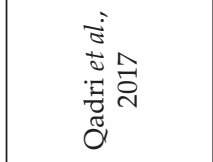 & 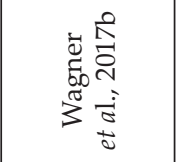 & 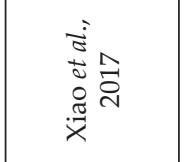 & 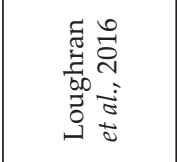 & 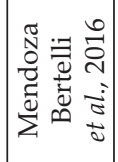 & 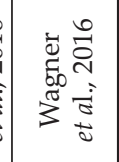 \\
\hline
\end{tabular}




\begin{tabular}{|c|c|c|c|c|c|c|c|c|c|c|c|}
\hline 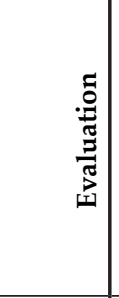 & 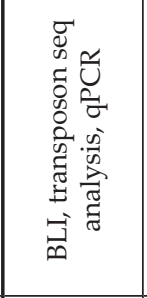 & 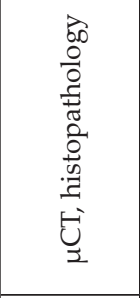 & 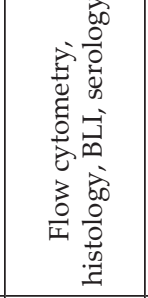 & 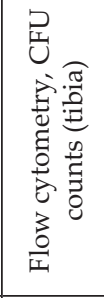 & 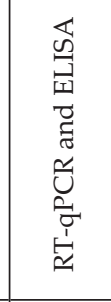 & 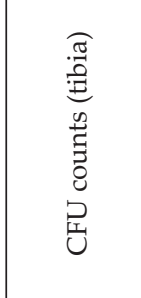 & 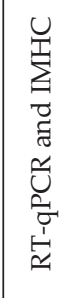 & 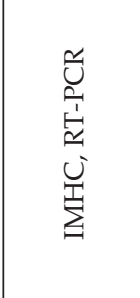 & 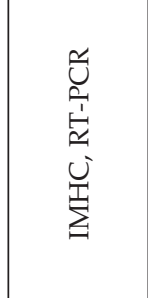 & 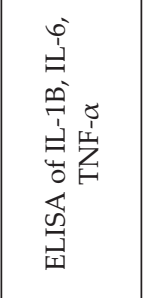 & 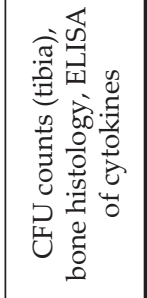 \\
\hline 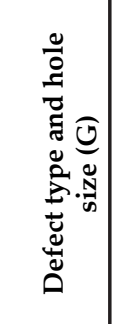 & 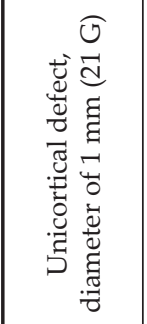 & 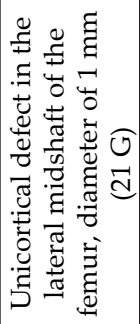 & 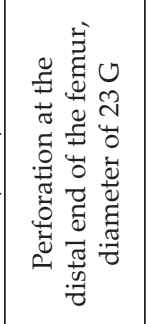 & 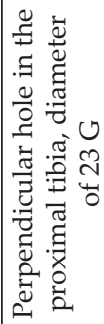 & & 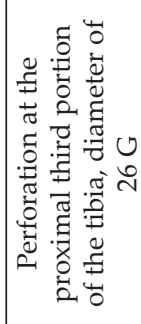 & & 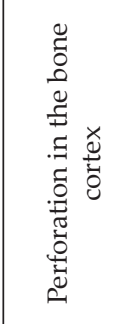 & 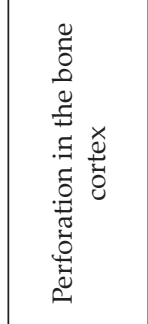 & 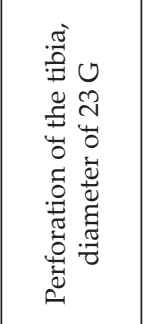 & 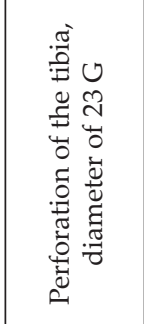 \\
\hline 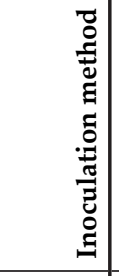 & 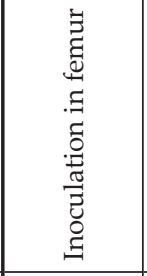 & 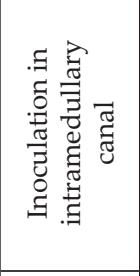 & 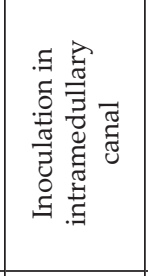 & 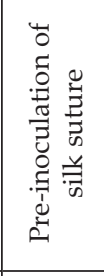 & 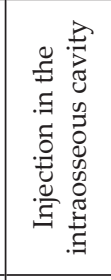 & 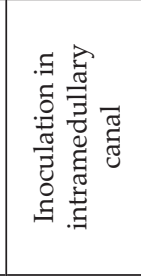 & 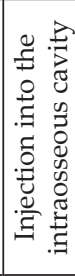 & 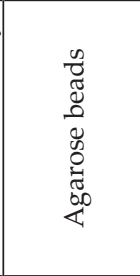 & 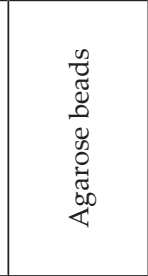 & 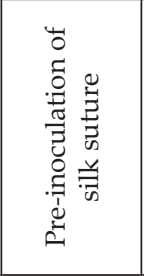 & 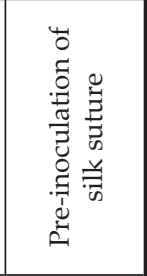 \\
\hline 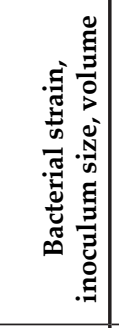 & 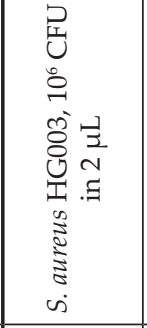 & 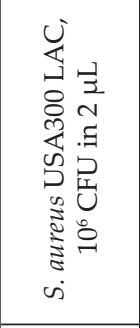 & 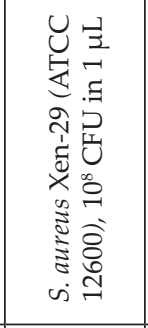 & 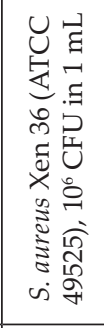 & 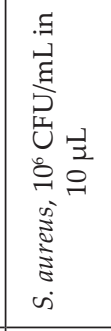 & 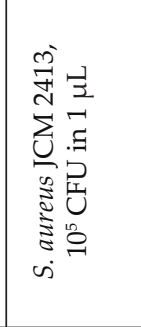 & 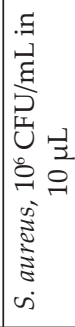 & 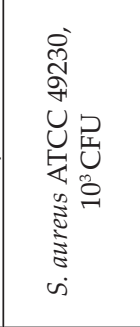 & 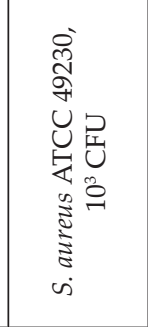 & 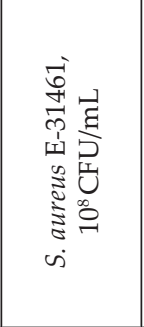 & 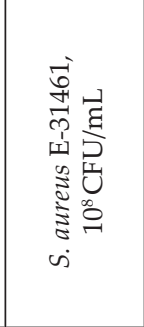 \\
\hline 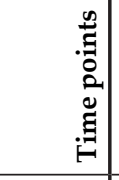 & & $\underset{I}{I}$ & 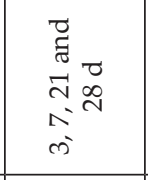 & $\stackrel{\Xi}{\circ}$ & $\underset{ }{\stackrel{I}{ }}$ & & $\underset{\mathbb{I}}{\stackrel{I}{I}}$ & $\begin{array}{l}\tilde{J} \\
\text { N } \\
\widetilde{\Xi} \\
\text { త్ }\end{array}$ & $\begin{array}{l}\tilde{J} \\
+ \\
\tilde{J} \\
\tilde{J} \\
N\end{array}$ & 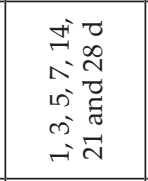 & 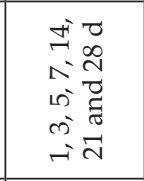 \\
\hline$\stackrel{8}{4}$ & 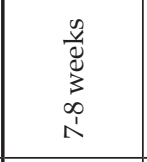 & 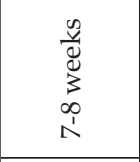 & 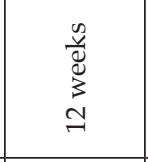 & 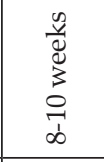 & 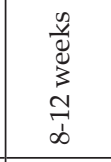 & 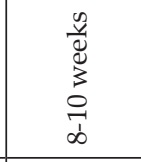 & 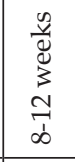 & ' & ' & $\begin{array}{l}n \\
\stackrel{n}{0} \\
\vdots \\
\vdots \\
n\end{array}$ & 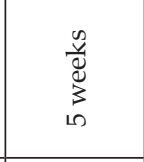 \\
\hline 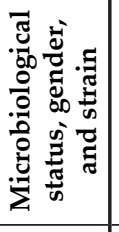 & 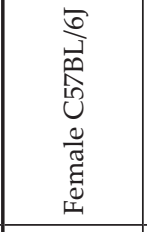 & $\begin{array}{l}\overrightarrow{0} \\
\stackrel{0}{0} \\
0 \\
0 \\
0\end{array}$ & 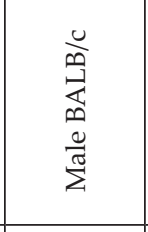 & 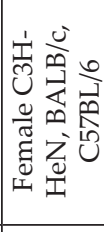 & 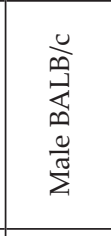 & 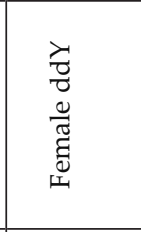 & $\begin{array}{l}\frac{u}{\infty} \\
\frac{\infty}{1} \\
\frac{0}{\pi} \\
\sum^{\pi}\end{array}$ & 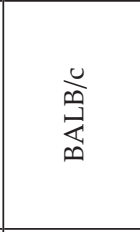 & 峁 & 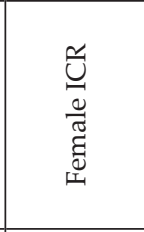 & 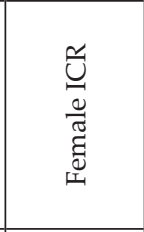 \\
\hline 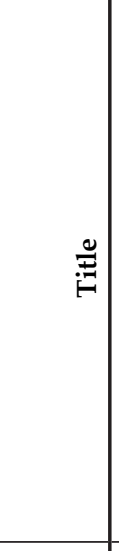 & 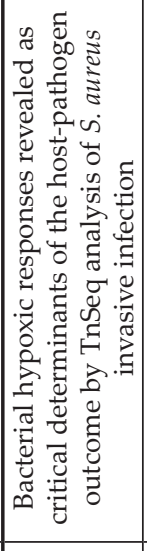 & 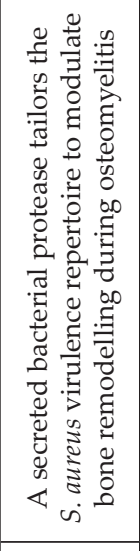 & 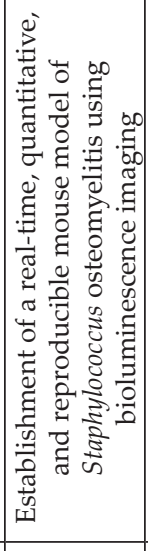 & 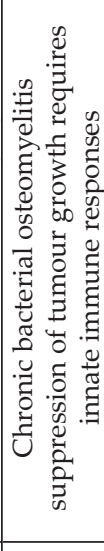 & 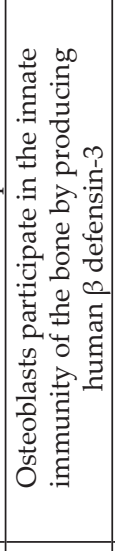 & 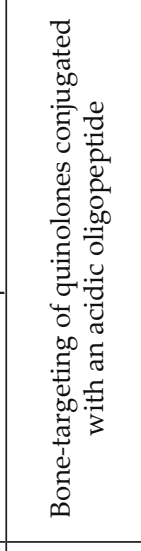 & 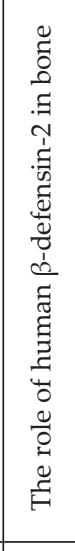 & 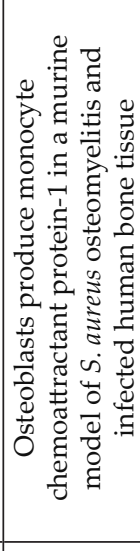 & 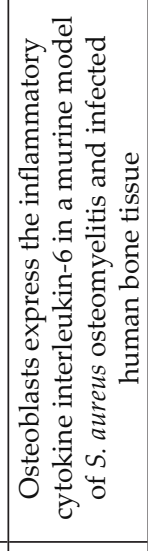 & 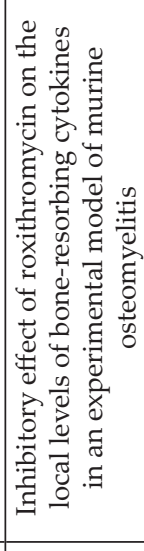 & 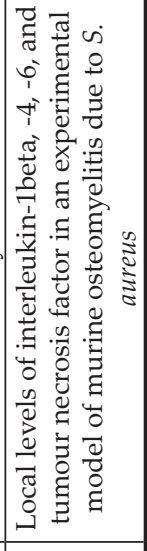 \\
\hline 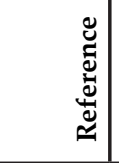 & 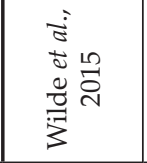 & 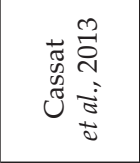 & 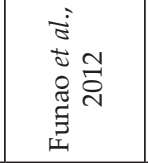 & 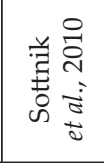 & 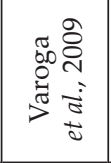 & 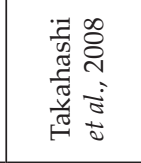 & 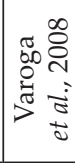 & 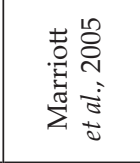 & 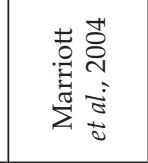 & 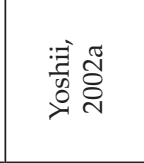 & 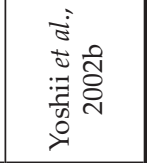 \\
\hline
\end{tabular}




\begin{tabular}{|c|c|c|c|c|c|c|c|c|c|c|c|}
\hline 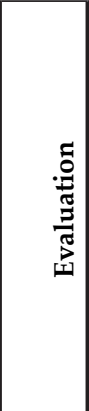 & 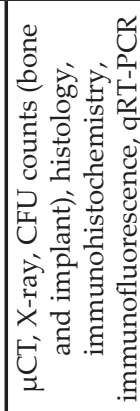 & 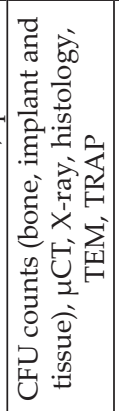 & 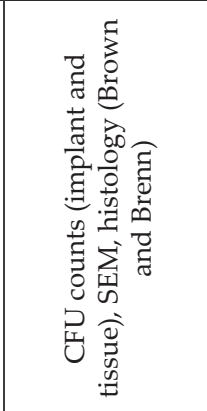 & 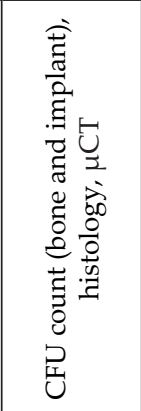 & 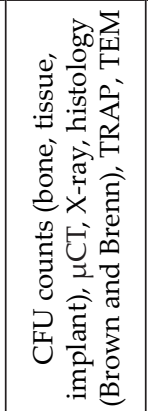 & 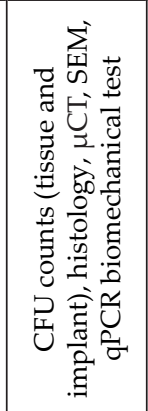 & 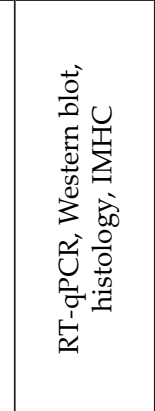 & 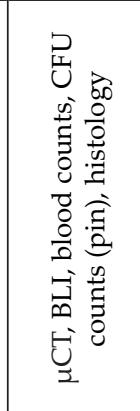 & 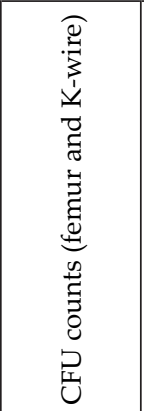 & 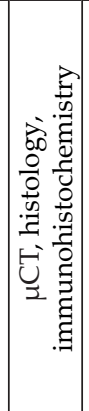 & 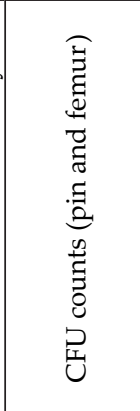 \\
\hline 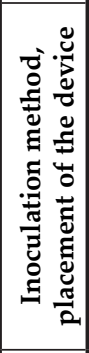 & 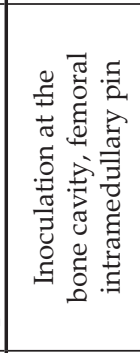 & 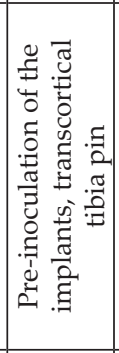 & 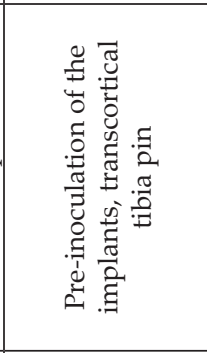 & 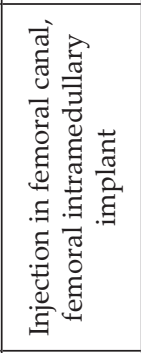 & 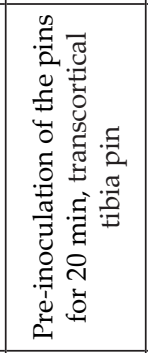 & 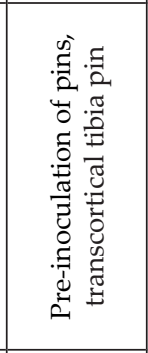 & 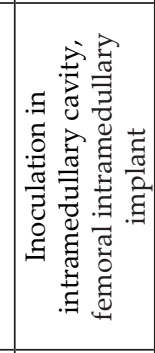 & 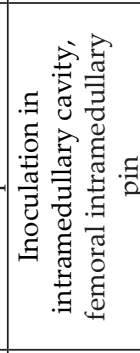 & 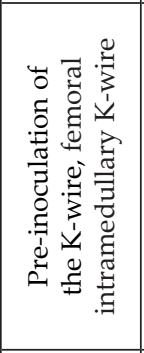 & 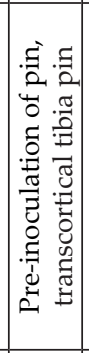 & 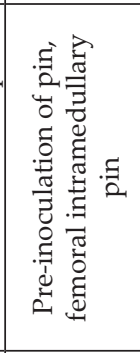 \\
\hline 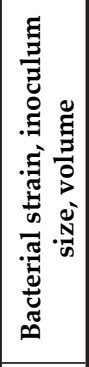 & 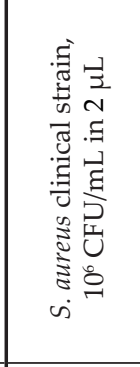 & 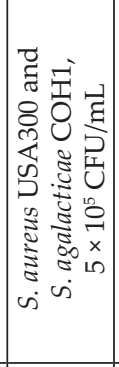 & 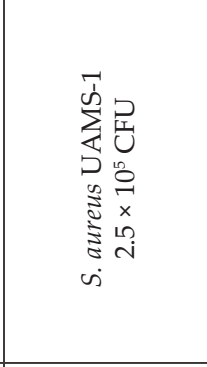 & 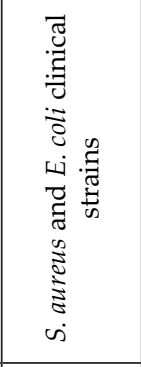 & 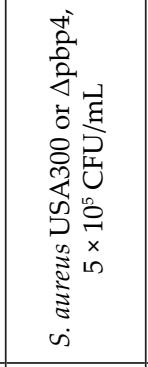 & 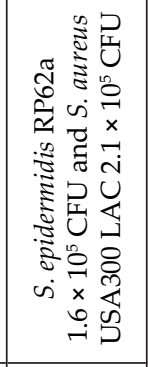 & 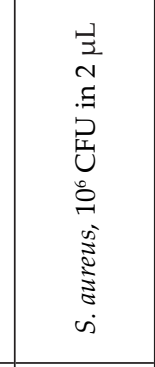 & 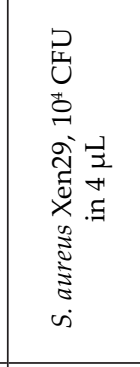 & 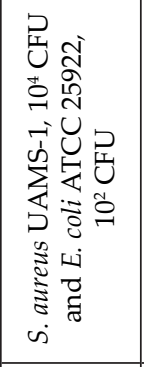 & 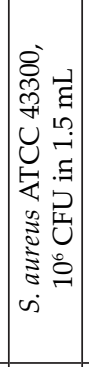 & 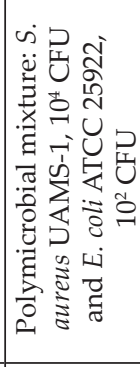 \\
\hline 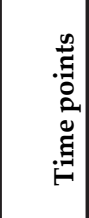 & 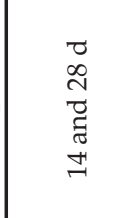 & $\begin{array}{l}J \\
\pm\end{array}$ & 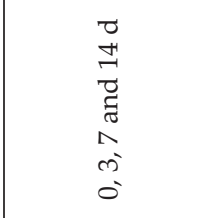 & $\begin{array}{l}\overrightarrow{0} \\
\stackrel{m}{m}\end{array}$ & $\begin{array}{l}\tilde{J} \\
\dot{J}\end{array}$ & 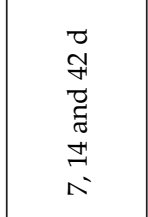 & $\vec{n}$ & 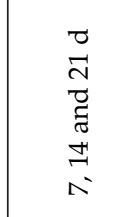 & $\tilde{r}$ & $\begin{array}{l}\overrightarrow{7} \\
F\end{array}$ & \\
\hline$\underset{4}{8}$ & 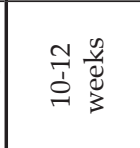 & $\begin{array}{l}\frac{n}{0} \\
\frac{\tilde{\nu}}{3} \\
0\end{array}$ & 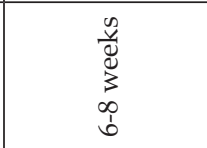 & $\begin{array}{l}\frac{n}{\tilde{g}} \\
\frac{\tilde{d}}{3} \\
\underline{0}\end{array}$ & 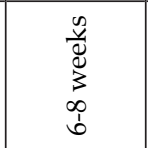 & 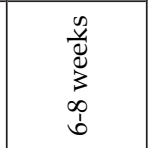 & $\begin{array}{l}\frac{n}{v} \\
\mathbb{u} \\
\vdots \\
\infty\end{array}$ & $\begin{array}{l}\simeq \\
\infty \\
\infty \\
\infty\end{array}$ & 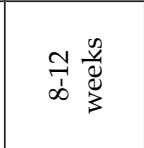 & $\begin{array}{l}\frac{v}{0} \\
\frac{0}{0} \\
3_{\infty}\end{array}$ & 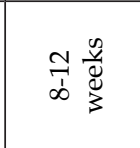 \\
\hline 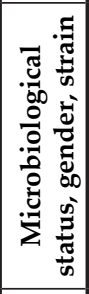 & 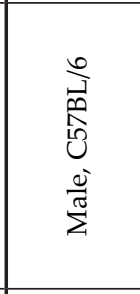 & 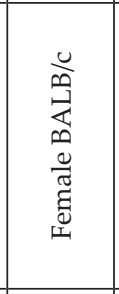 & 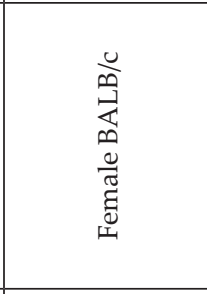 & $\begin{array}{l}\overrightarrow{\tilde{\theta}} \\
\frac{\tilde{u}}{\sigma} \\
\vec{\pi}\end{array}$ & 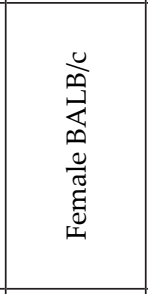 & 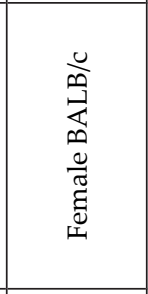 & 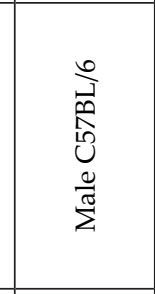 & 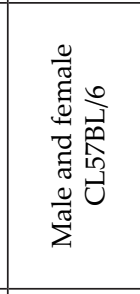 & $\begin{array}{l}\stackrel{0}{\vec{A}} \\
\stackrel{0}{0} \\
b \\
0\end{array}$ & 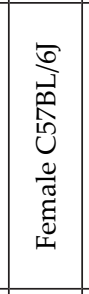 & $\begin{array}{l}\stackrel{0}{\overrightarrow{7}} \\
\stackrel{0}{0} \\
0 \\
0\end{array}$ \\
\hline$\stackrel{\varrho}{\underline{\Xi}}$ & 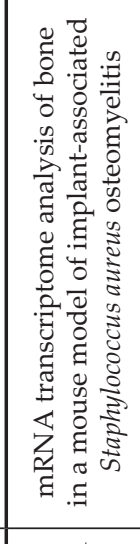 & 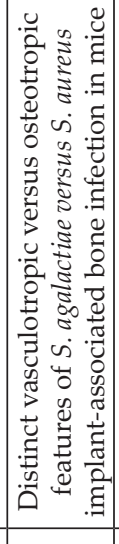 & 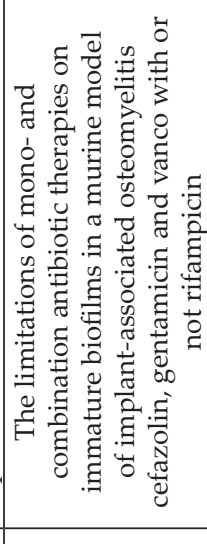 & 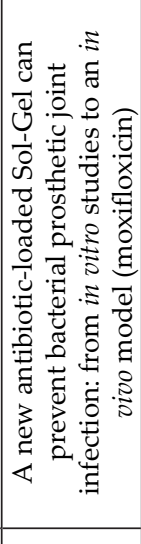 & 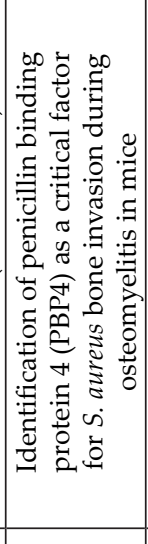 & 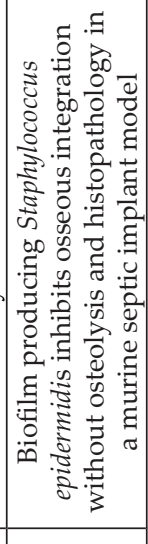 & 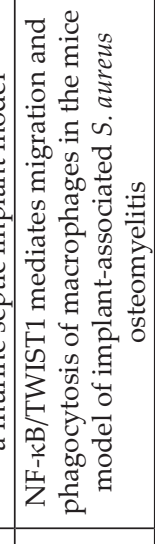 & 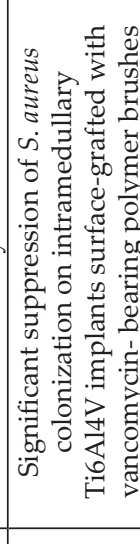 & 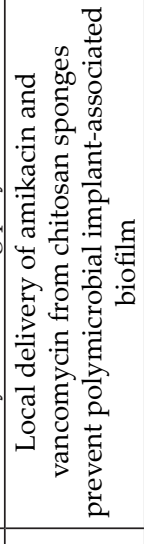 & 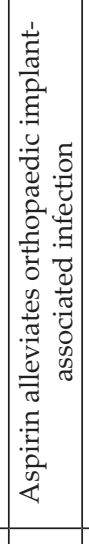 & 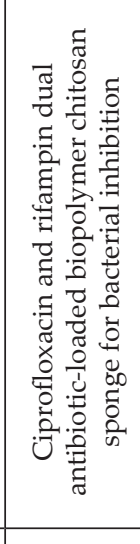 \\
\hline 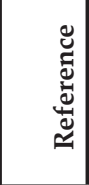 & 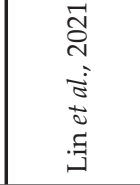 & 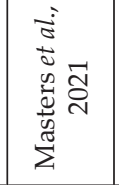 & 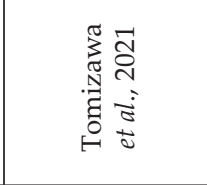 & 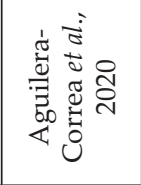 & 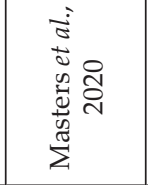 & 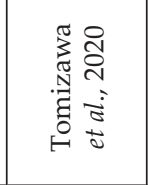 & 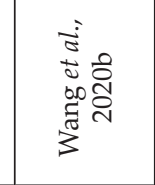 & 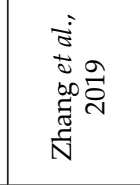 & 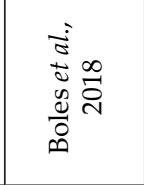 & 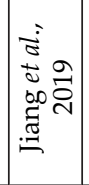 & 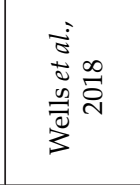 \\
\hline
\end{tabular}




\begin{tabular}{|c|c|c|c|c|c|c|c|c|c|c|}
\hline 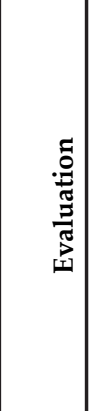 & 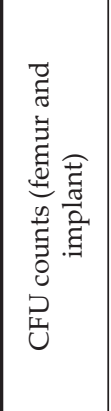 & 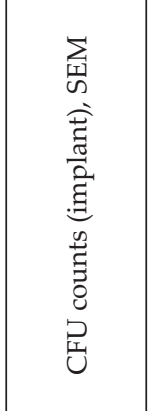 & 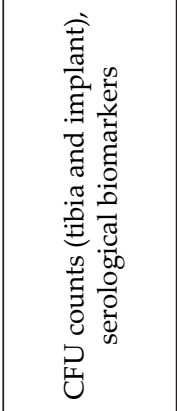 & 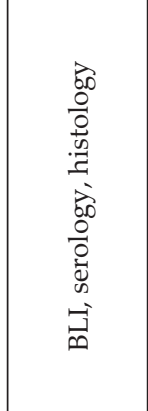 & 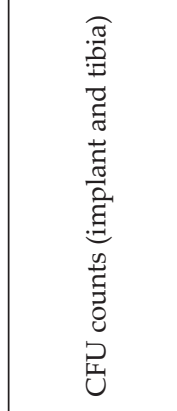 & 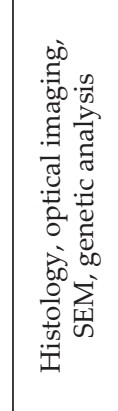 & 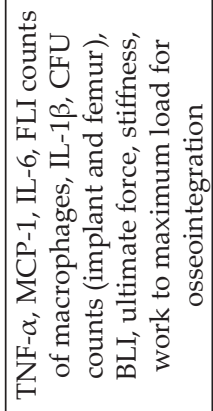 & 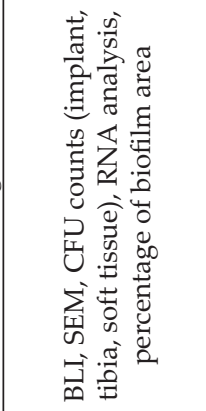 & 煦 & 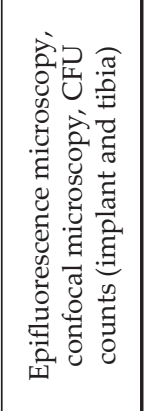 \\
\hline 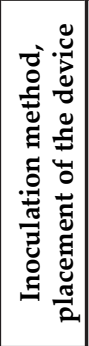 & 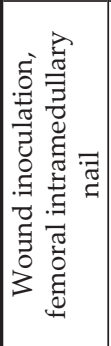 & 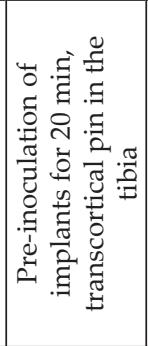 & 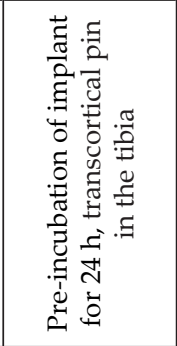 & 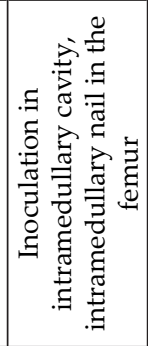 & 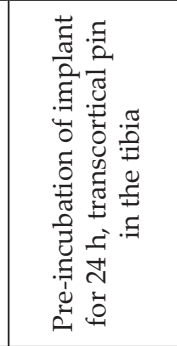 & 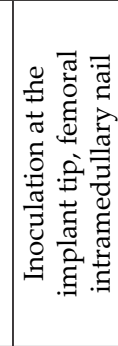 & 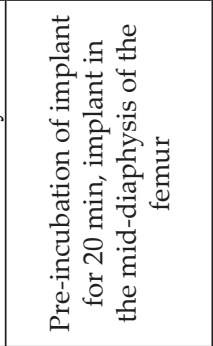 & 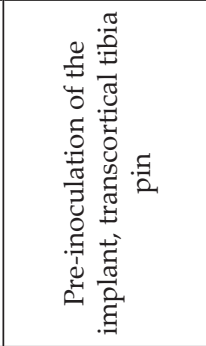 & 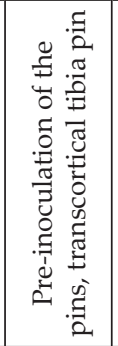 & 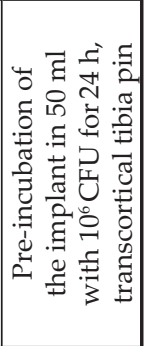 \\
\hline 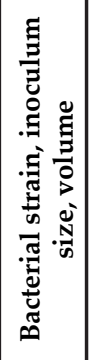 & 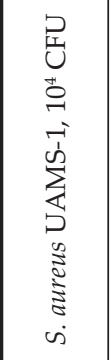 & 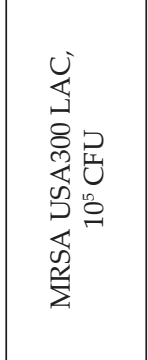 & 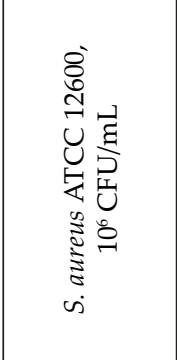 & 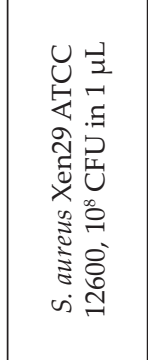 & 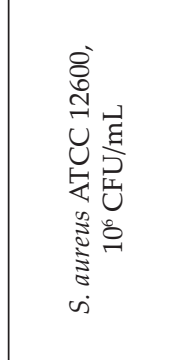 & 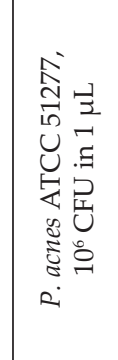 & 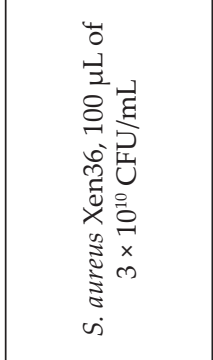 & 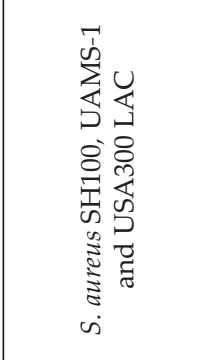 & 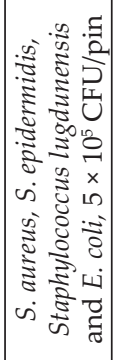 & 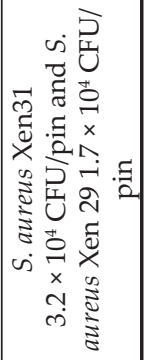 \\
\hline 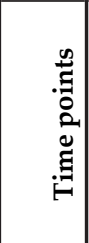 & $\bar{\nabla}$ & 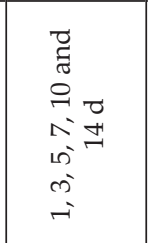 & $\begin{array}{l}\tilde{I} \\
\dot{H}\end{array}$ & $\begin{array}{l}\vec{D} \\
\stackrel{\sim}{ }\end{array}$ & 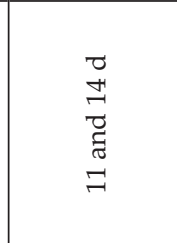 & 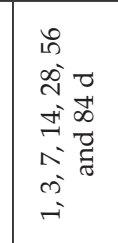 & $\begin{array}{l}\vec{\Xi} \\
\stackrel{1}{7} \\
\vec{\Xi} \\
\vec{\sigma} \\
-\end{array}$ & 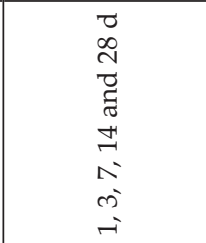 & 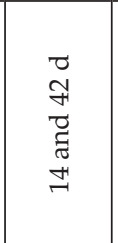 & $\begin{array}{l}\overrightarrow{0} \\
0 \\
\overrightarrow{7} \\
\overrightarrow{0} \\
0 \\
ت \\
= \\
\infty \\
0 \\
0 \\
+1\end{array}$ \\
\hline$\underset{8}{\alpha}$ & 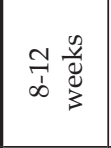 & 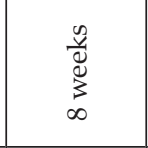 & 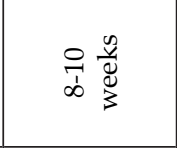 & 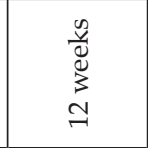 & 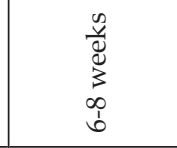 & 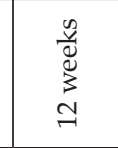 & $\begin{array}{ll}\infty & 0 \\
0 & \frac{v}{0} \\
0 & 0 \\
0 & 3\end{array}$ & & 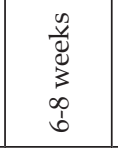 & 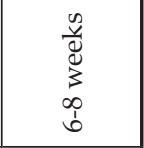 \\
\hline 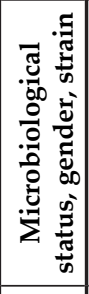 & 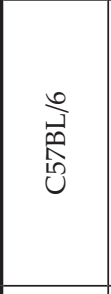 & 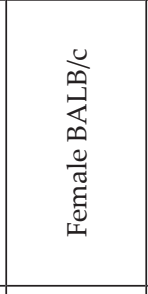 & 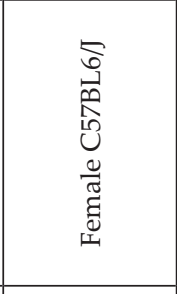 & 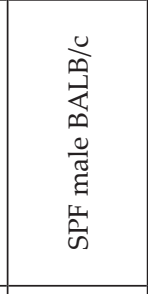 & 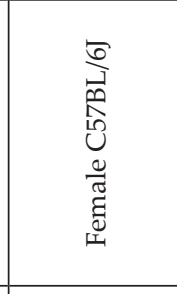 & 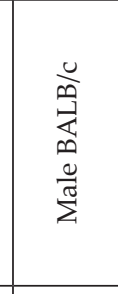 & 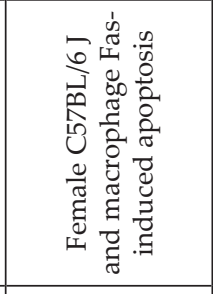 & 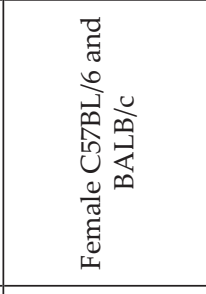 & 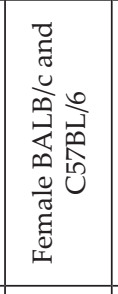 & 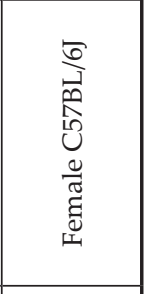 \\
\hline 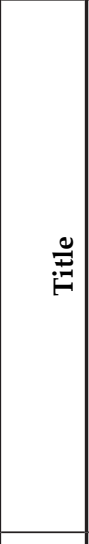 & 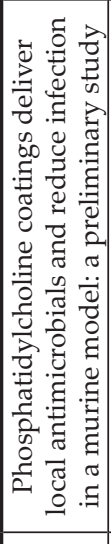 & 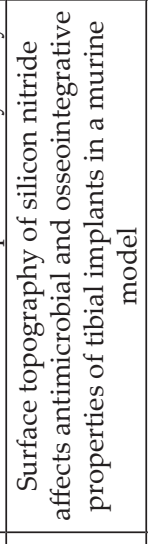 & 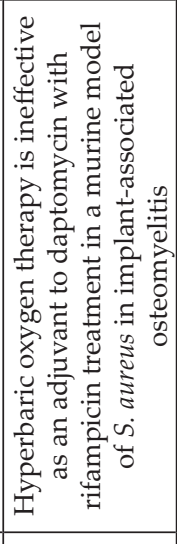 & 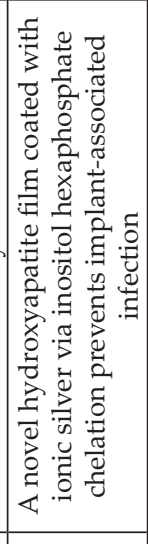 & 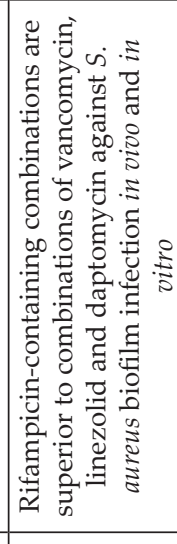 & 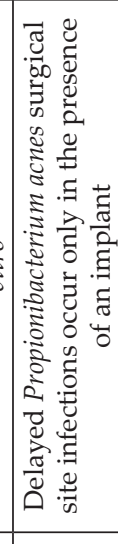 & 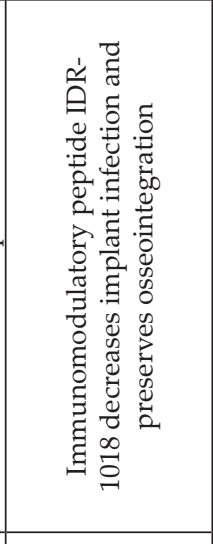 & 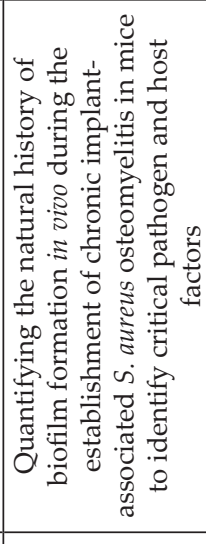 & 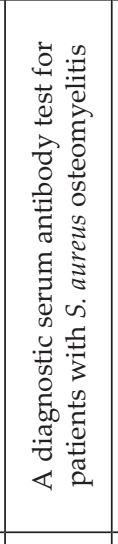 & 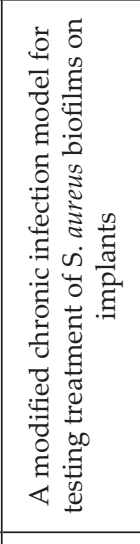 \\
\hline 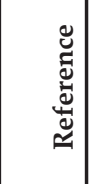 & 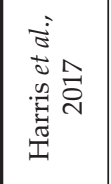 & 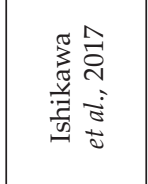 & 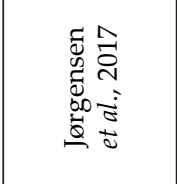 & 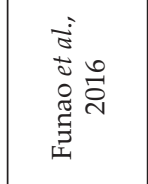 & 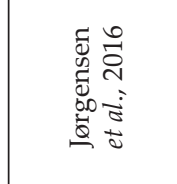 & 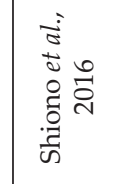 & 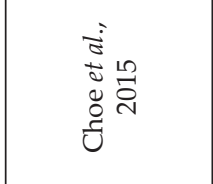 & 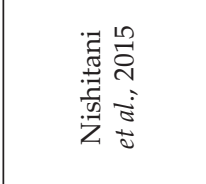 & 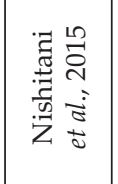 & 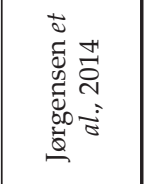 \\
\hline
\end{tabular}




\begin{tabular}{|c|c|c|c|c|c|c|c|c|c|c|}
\hline 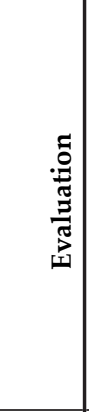 & 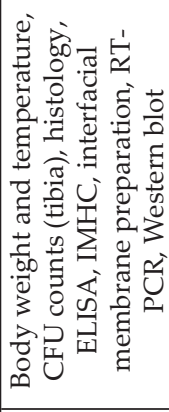 & 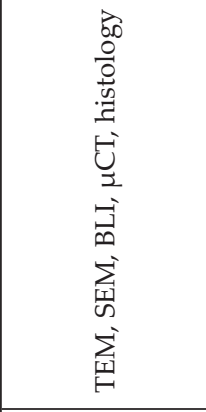 & 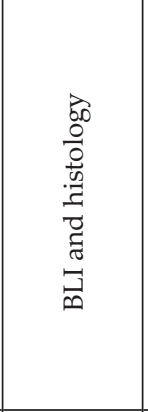 & 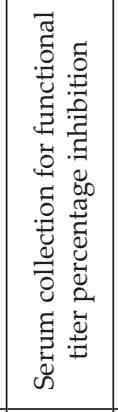 & 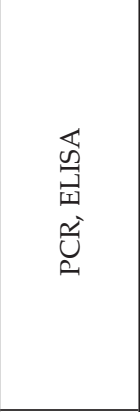 & 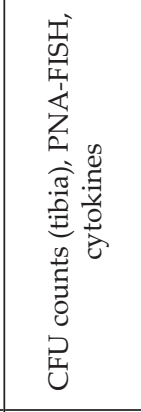 & 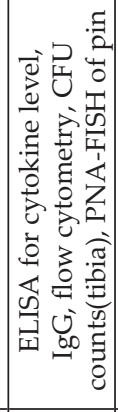 & 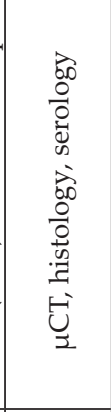 & 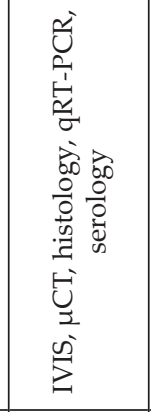 & 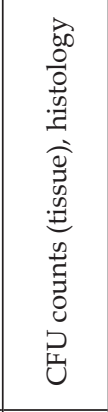 \\
\hline 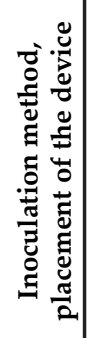 & 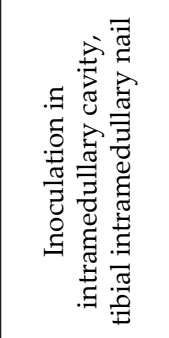 & 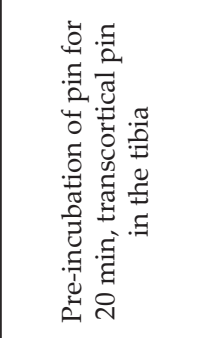 & 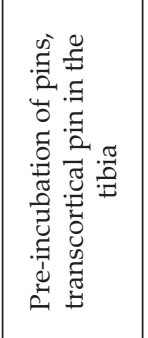 & 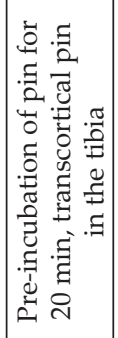 & 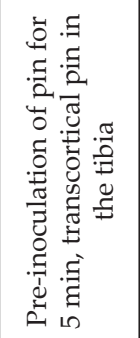 & 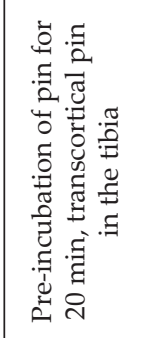 & 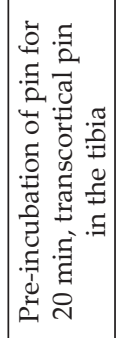 & 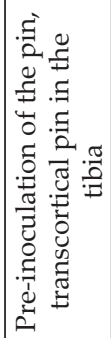 & 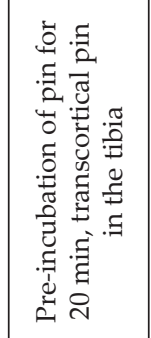 & 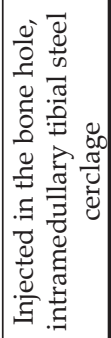 \\
\hline 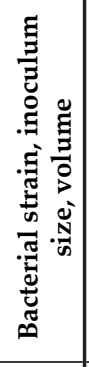 & 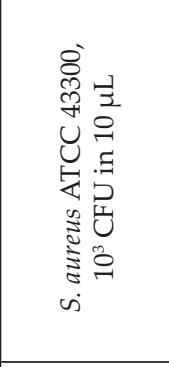 & 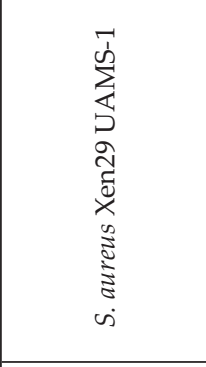 & 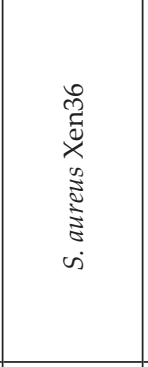 & 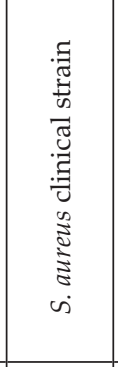 & 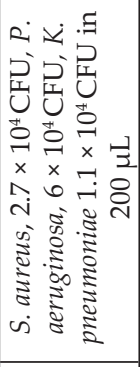 & 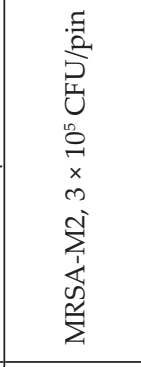 & 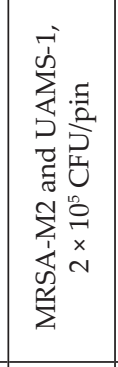 & 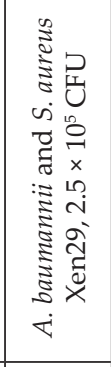 & 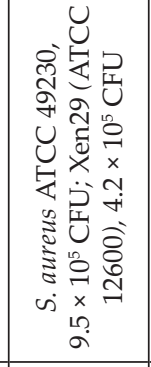 & 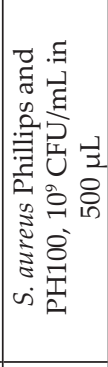 \\
\hline 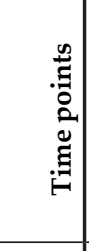 & 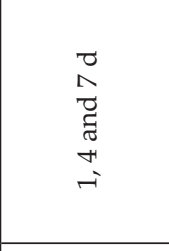 & 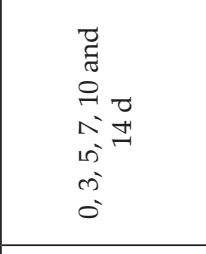 & 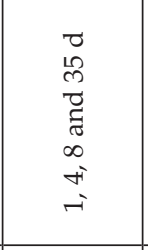 & $\vec{I}$ & 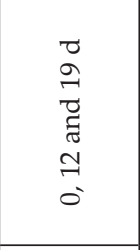 & 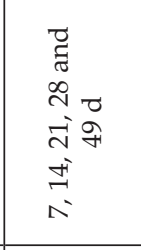 & 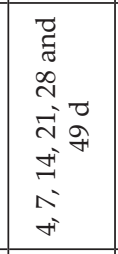 & $\begin{array}{l}\bar{\sigma} \\
\stackrel{2}{2}\end{array}$ & 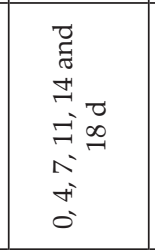 & $\begin{array}{l}\overrightarrow{0} \\
\widetilde{D} \\
\dot{d} \\
\text { id }\end{array}$ \\
\hline$\stackrel{8}{\alpha}$ & $\begin{array}{l}\cong \\
\cdots \\
\infty \\
\infty\end{array}$ & 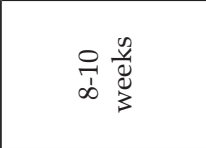 & 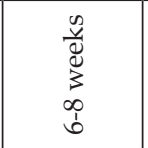 & 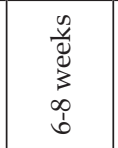 & 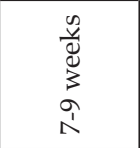 & 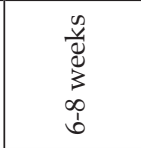 & 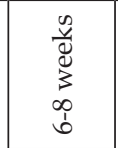 & 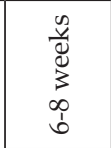 & 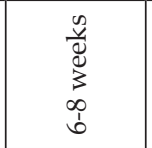 & 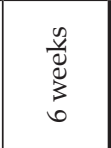 \\
\hline 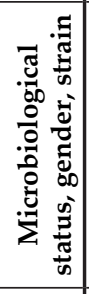 & 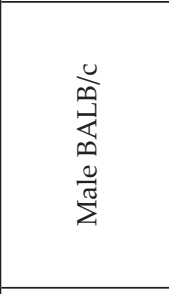 & 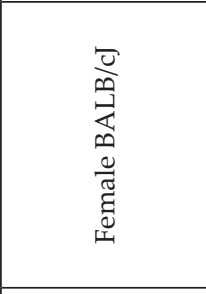 & 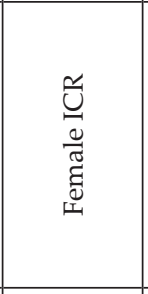 & 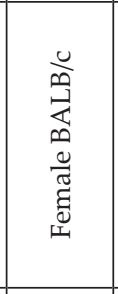 & $\begin{array}{l}0 \\
د \\
0 \\
0 \\
0 \\
0 \\
\frac{0}{\pi} \\
\Sigma\end{array}$ & 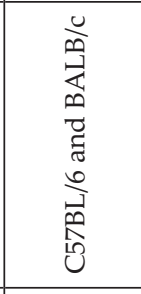 & 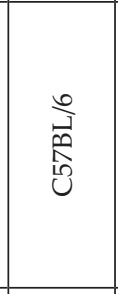 & 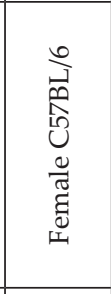 & 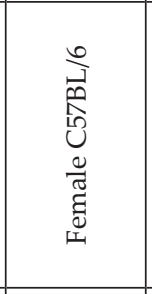 & 『ึ \\
\hline$\stackrel{\varrho}{\Xi}$ & 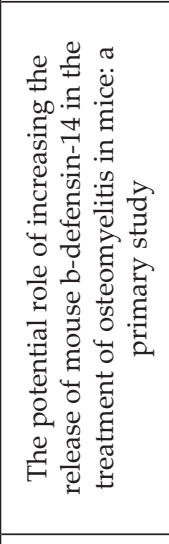 & 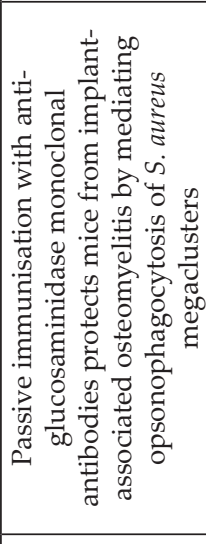 & 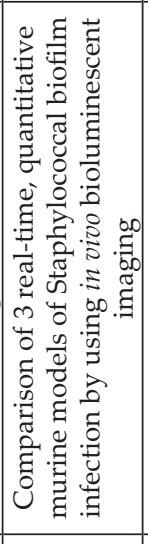 & 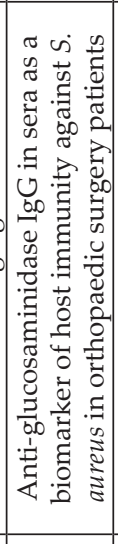 & 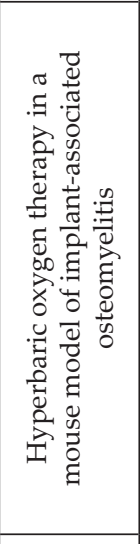 & 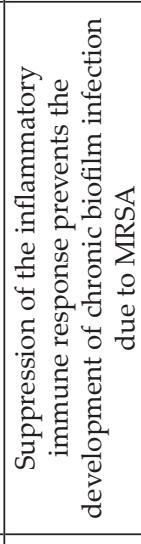 & 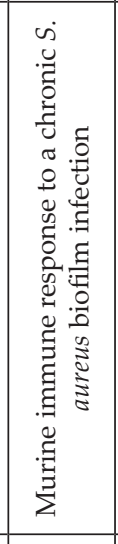 & 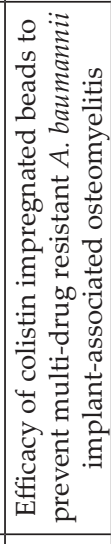 & 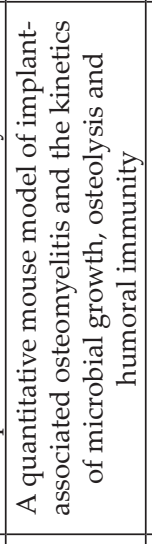 & 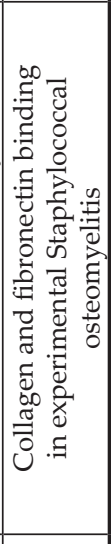 \\
\hline 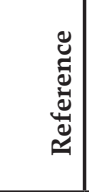 & 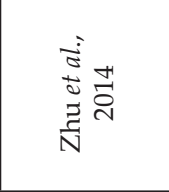 & 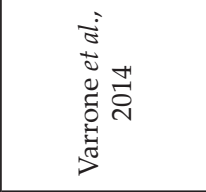 & 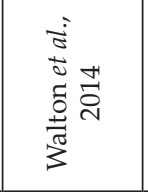 & 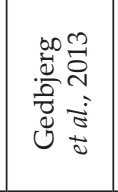 & 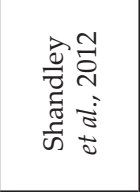 & 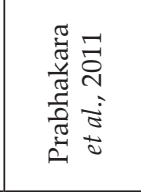 & 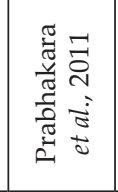 & 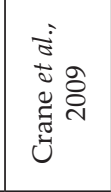 & 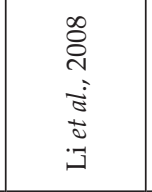 & 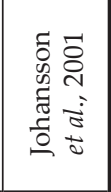 \\
\hline
\end{tabular}




\begin{tabular}{|c|c|c|c|c|c|c|}
\hline 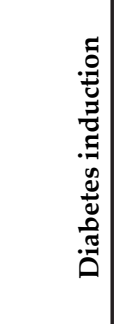 & 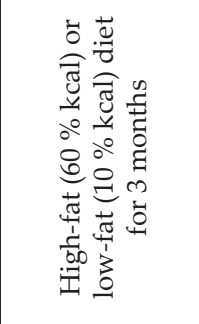 & 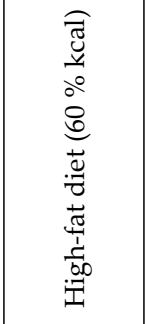 & 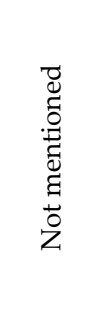 & 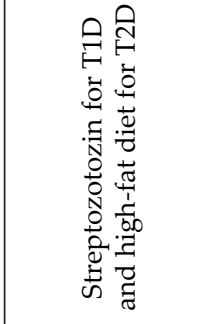 & 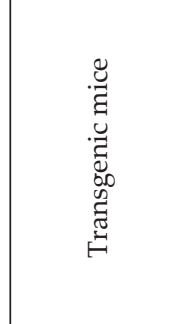 & 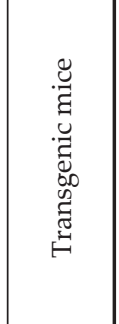 \\
\hline 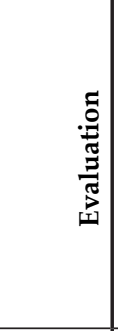 & 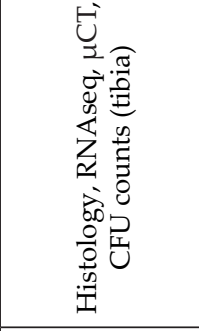 & 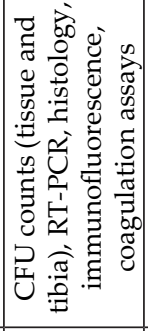 & 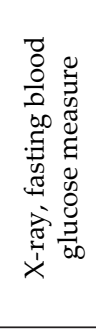 & 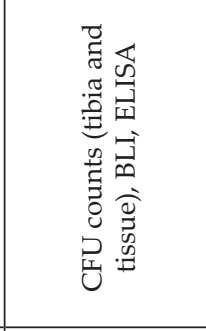 & 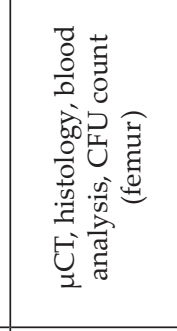 & 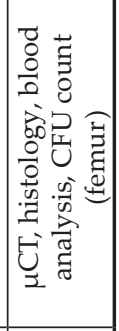 \\
\hline 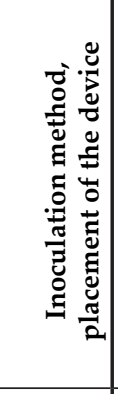 & 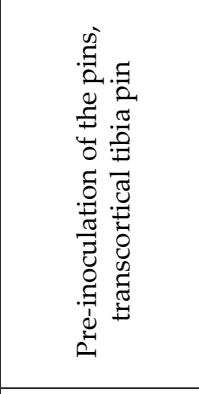 & 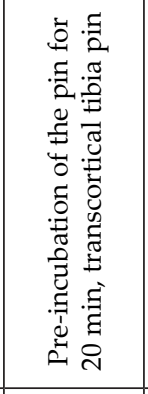 & 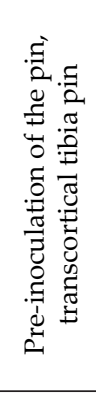 & 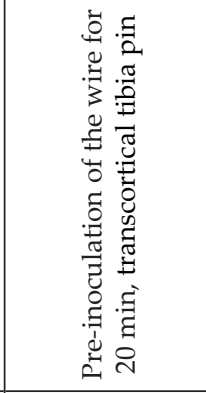 & 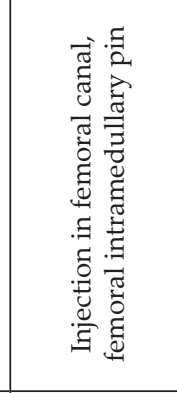 & 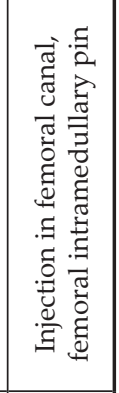 \\
\hline 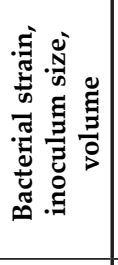 & 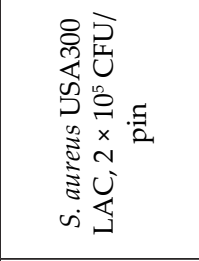 & 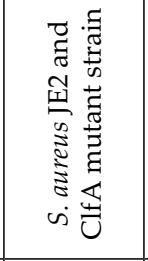 & 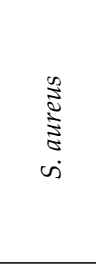 & 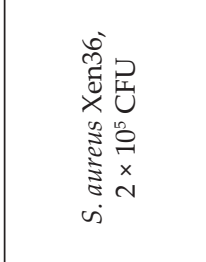 & 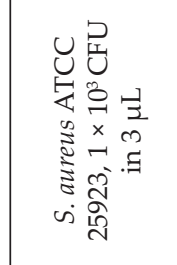 & 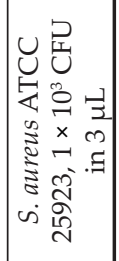 \\
\hline 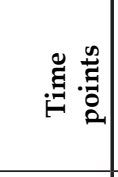 & 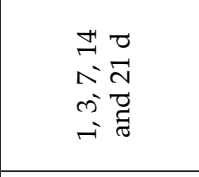 & $\begin{array}{l}\vec{J} \\
\vec{N} \\
\vec{J} \\
\vec{J} \\
\vec{J}\end{array}$ & & 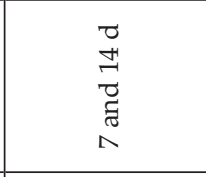 & 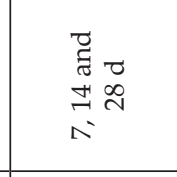 & $\begin{array}{l}\vec{D} \\
\underset{\sim}{\infty}\end{array}$ \\
\hline$\stackrel{8}{4}$ & & 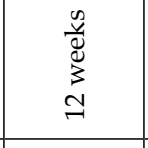 & 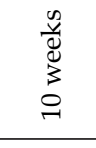 & 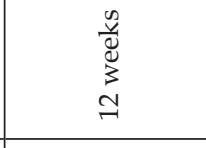 & 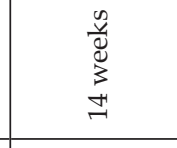 & \\
\hline 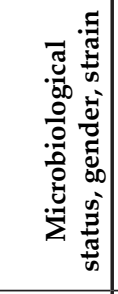 & 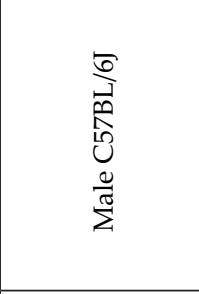 & 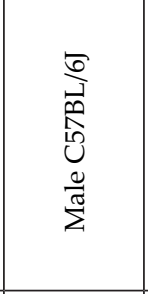 & 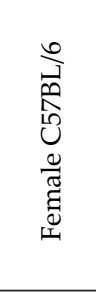 & 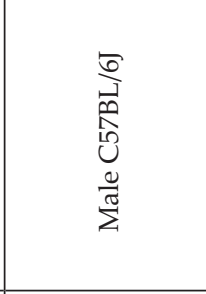 & 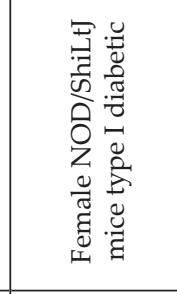 & 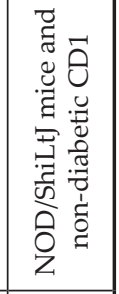 \\
\hline 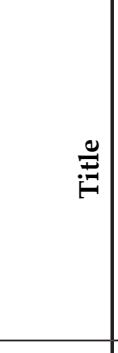 & 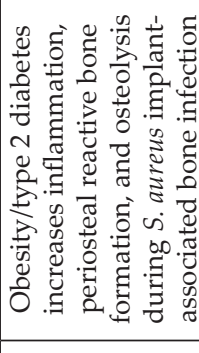 & 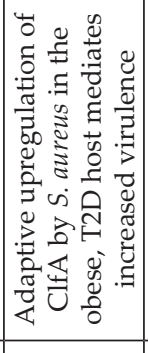 & 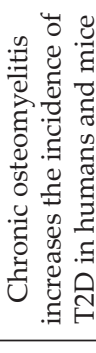 & 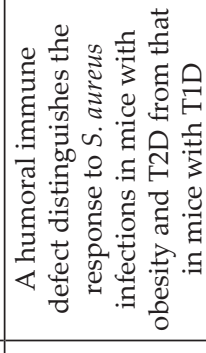 & 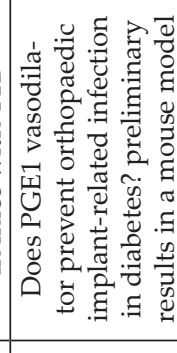 & 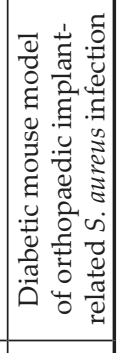 \\
\hline 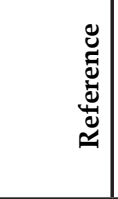 & 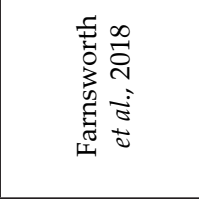 & 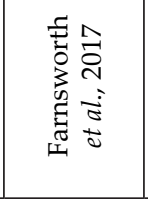 & 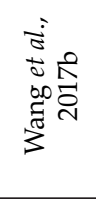 & 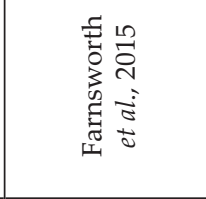 & 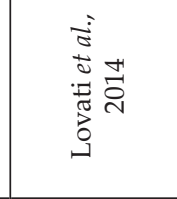 & 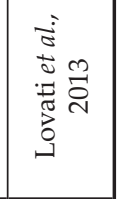 \\
\hline
\end{tabular}




\begin{tabular}{|c|c|c|c|c|c|c|c|c|c|c|c|c|c|}
\hline 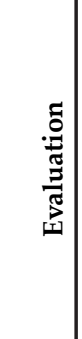 & 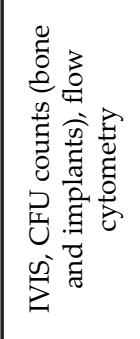 & 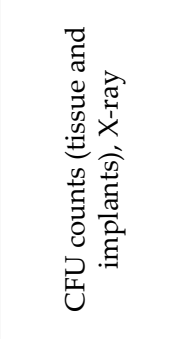 & 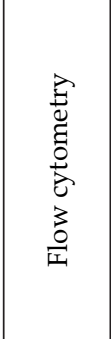 & 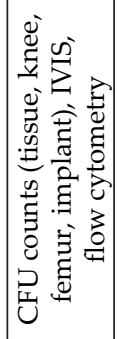 & 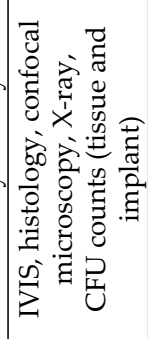 & 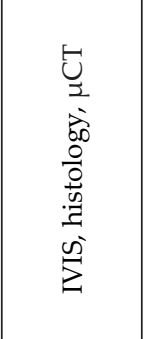 & 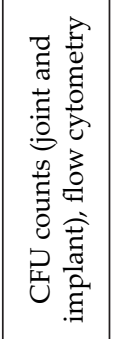 & 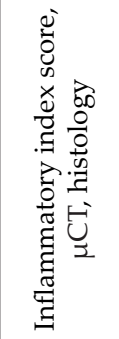 & 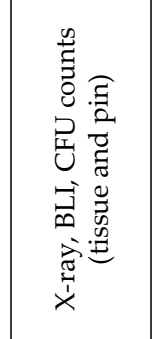 & 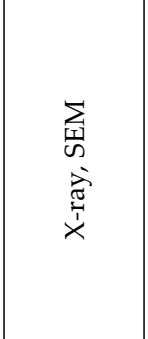 & 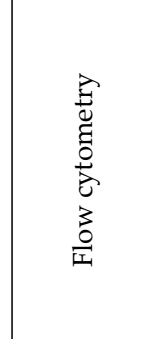 & 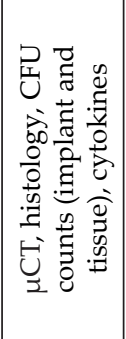 & 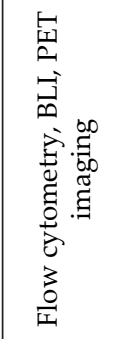 \\
\hline 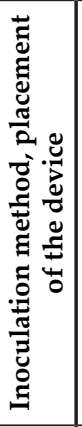 & 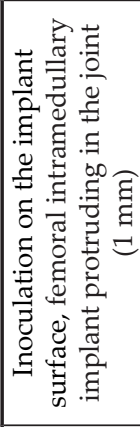 & 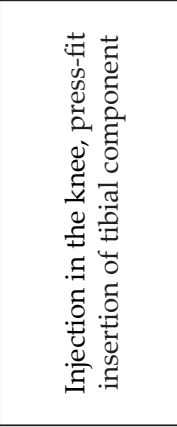 & 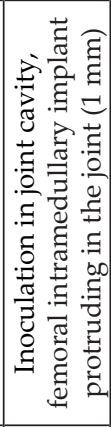 & 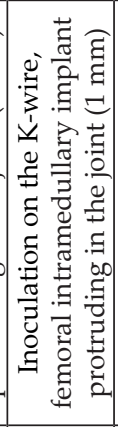 & 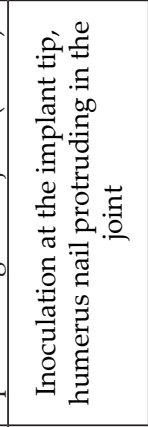 & 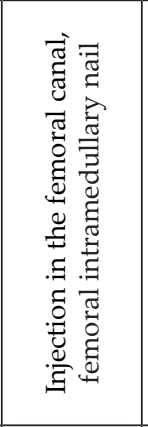 & 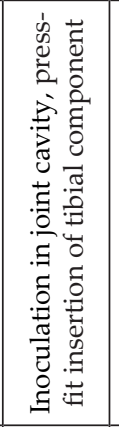 & 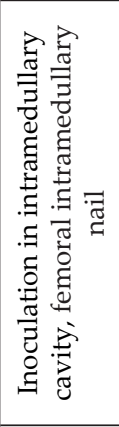 & 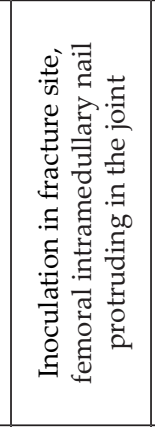 & 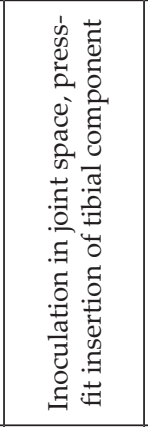 & 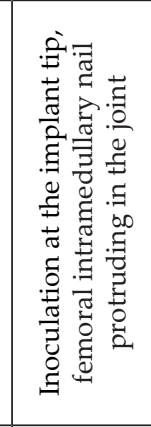 & 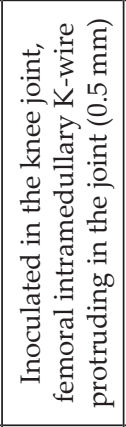 & 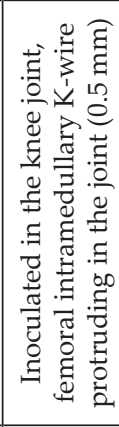 \\
\hline 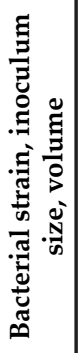 & 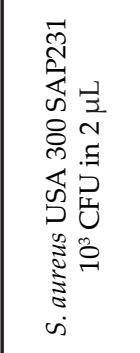 & 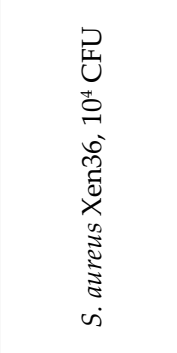 & 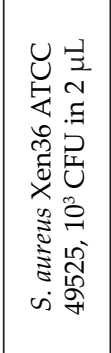 & 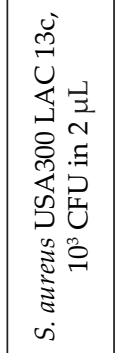 & 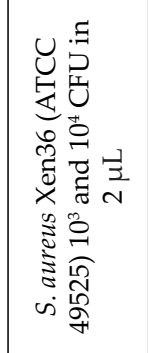 & 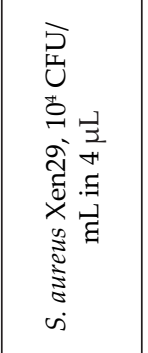 & 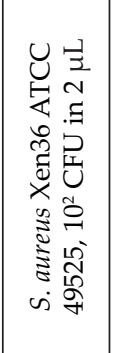 & 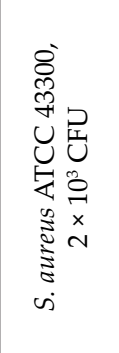 & 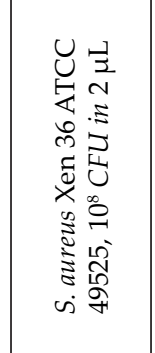 & 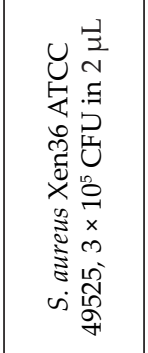 & 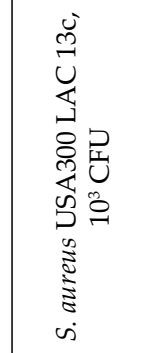 & 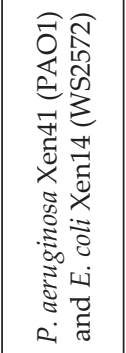 & 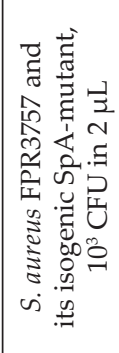 \\
\hline 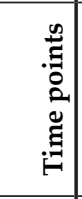 & 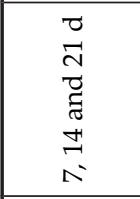 & $\stackrel{0}{\circ}$ & 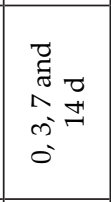 & 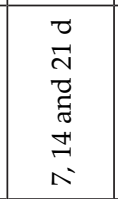 & 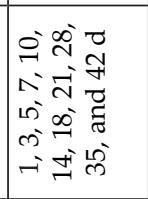 & $\overrightarrow{\tilde{N}}$ & in & $\bar{\Lambda}$ & 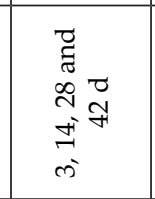 & J & 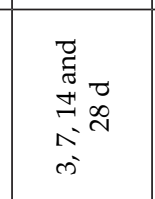 & 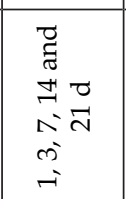 & 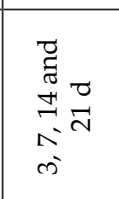 \\
\hline 品 & 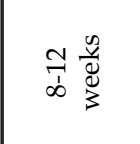 & 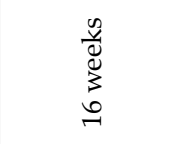 & 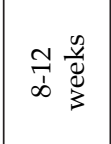 & 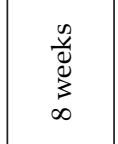 & 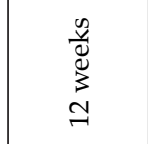 & 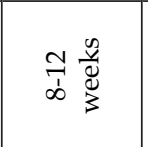 & 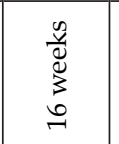 & 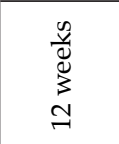 & $\begin{array}{l}\mathcal{N} \frac{\infty}{0} \\
\frac{0}{\pi} \\
\frac{\pi}{2}\end{array}$ & 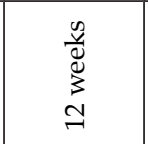 & 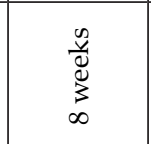 & 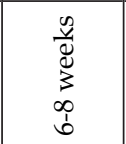 & $\begin{array}{l}\frac{n}{v} \\
\frac{\pi}{3} \\
3 \\
0\end{array}$ \\
\hline 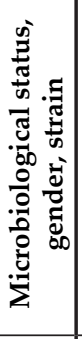 & $\begin{array}{l}\frac{\overrightarrow{0}}{7} \\
\frac{0}{0} \\
0 \\
0 \\
\frac{0}{\pi} \\
\frac{\pi}{2}\end{array}$ & 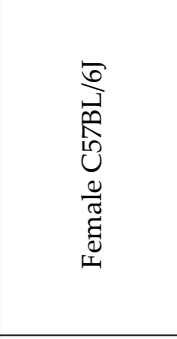 & 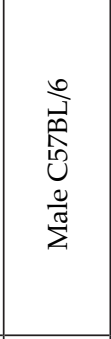 & 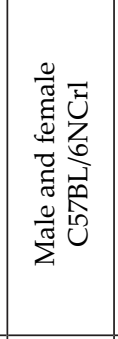 & 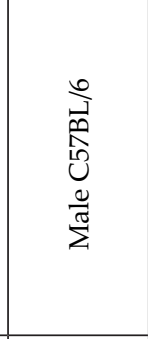 & 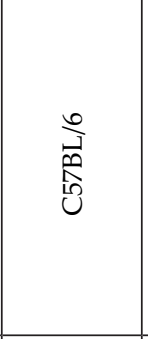 & 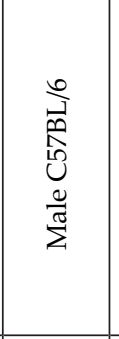 & 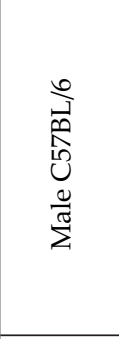 & 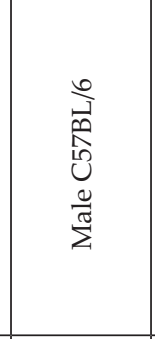 & 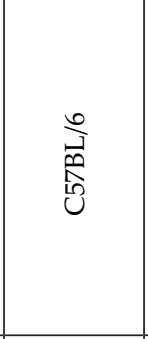 & 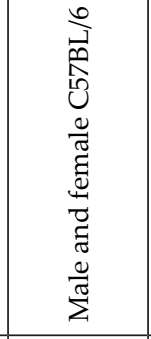 & 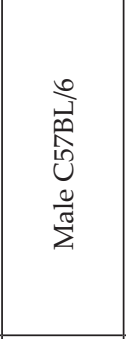 & 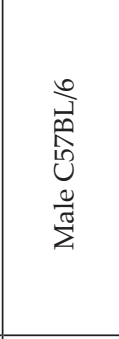 \\
\hline 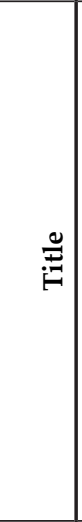 & 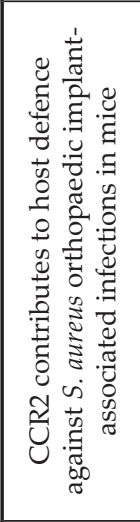 & 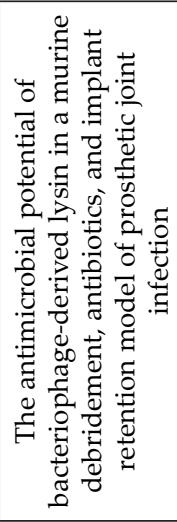 & 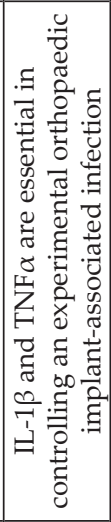 & 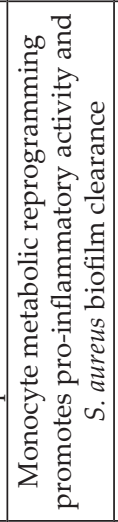 & 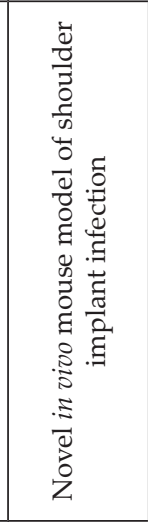 & 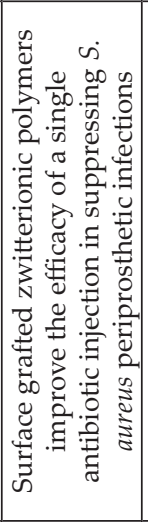 & 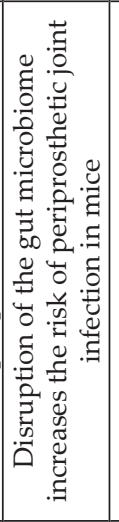 & 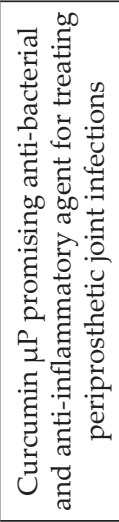 & 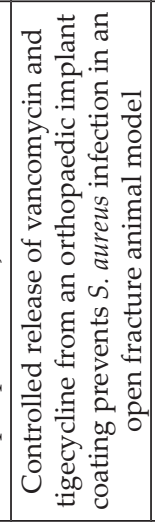 & 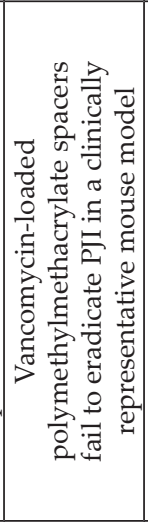 & 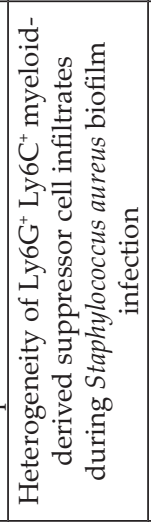 & 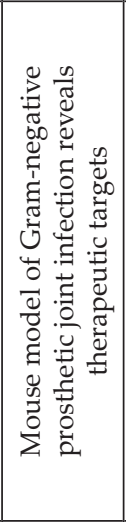 & 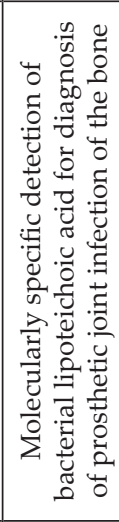 \\
\hline 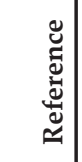 & 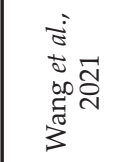 & 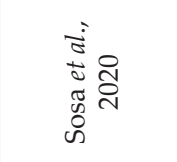 & 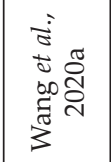 & 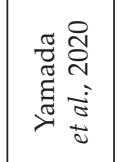 & 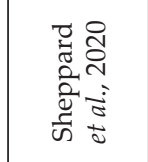 & 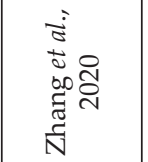 & 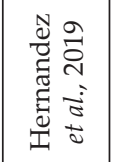 & 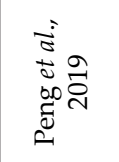 & 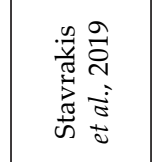 & 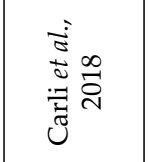 & 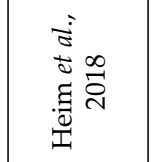 & 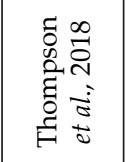 & 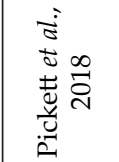 \\
\hline
\end{tabular}




\begin{tabular}{|c|c|c|c|c|c|c|c|c|c|c|c|c|}
\hline 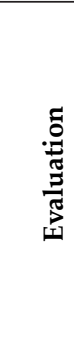 & 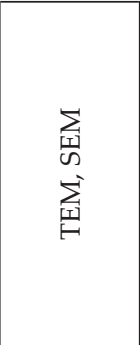 & 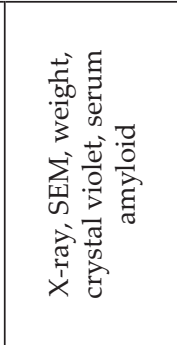 & $\sum^{m}$ & 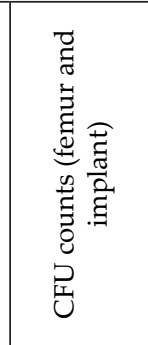 & 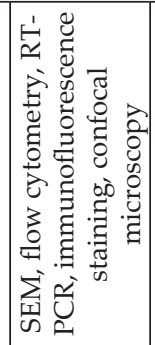 & 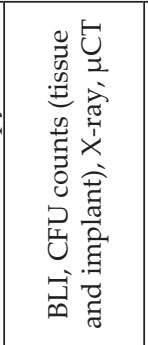 & $\begin{array}{l}\overrightarrow{\vec{I}} \\
\overrightarrow{\vec{D}}\end{array}$ & 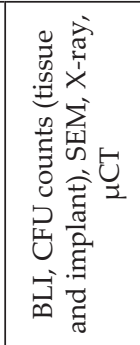 & 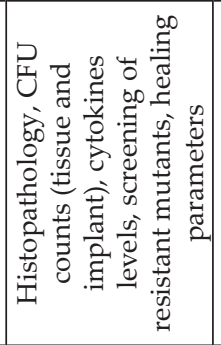 & 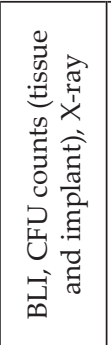 & 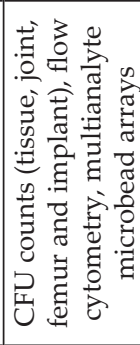 & 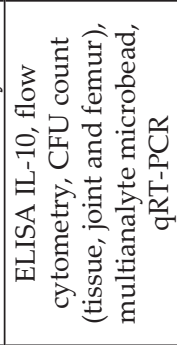 \\
\hline 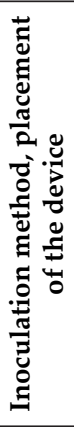 & 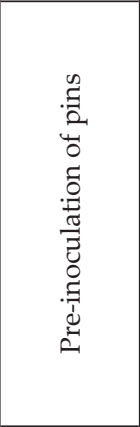 & 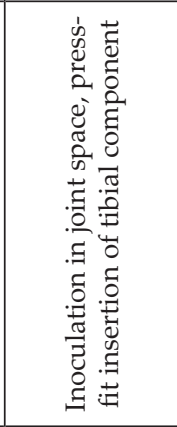 & 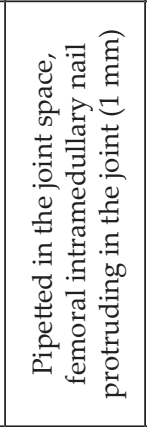 & 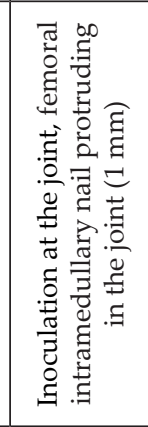 & 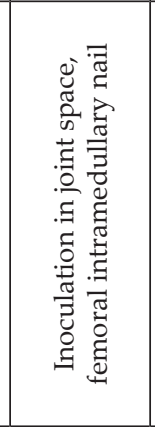 & 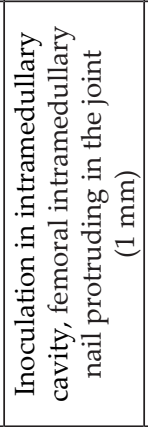 & 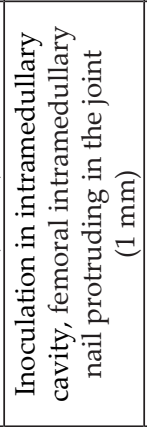 & 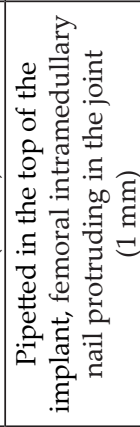 & 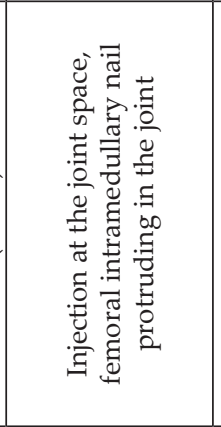 & 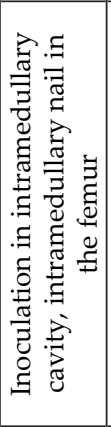 & 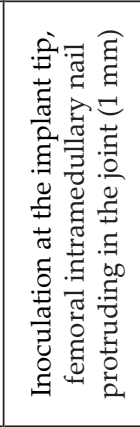 & 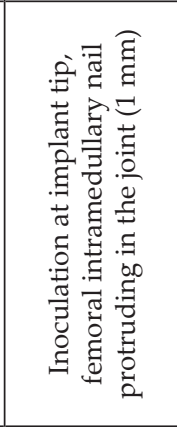 \\
\hline 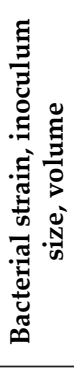 & 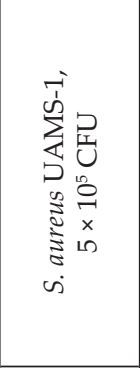 & 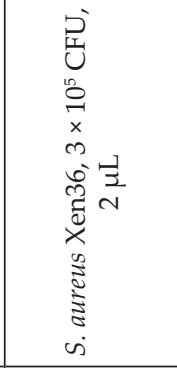 & 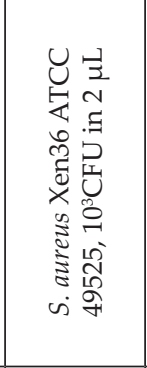 & 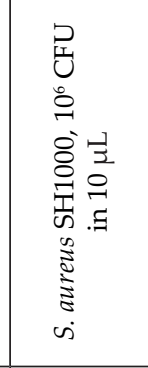 & 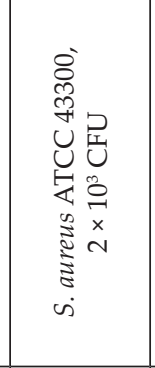 & 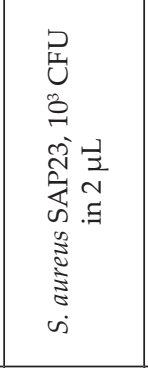 & 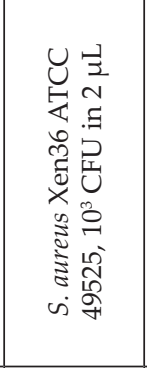 & 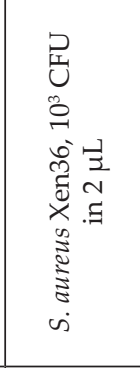 & 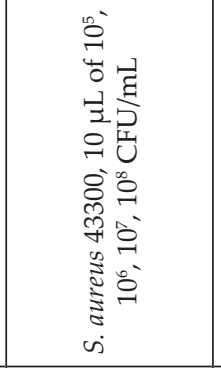 & 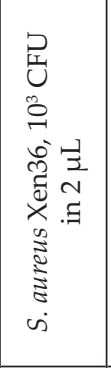 & 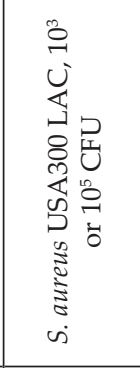 & 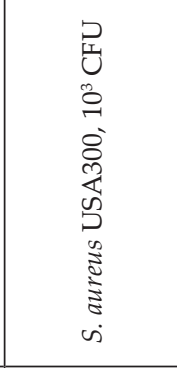 \\
\hline 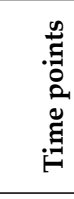 & $\begin{array}{l}\vec{J} \\
\vec{H}\end{array}$ & 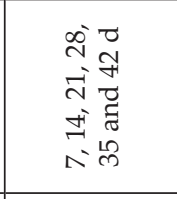 & & $\bar{m}$ & ${ }_{\infty}^{-}$ & 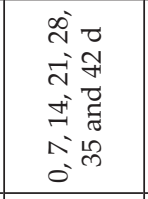 & 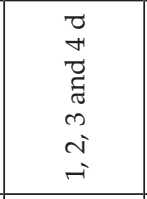 & 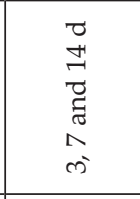 & 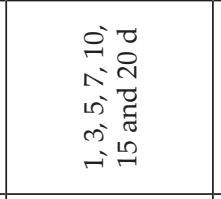 & 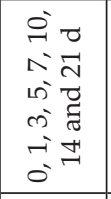 & 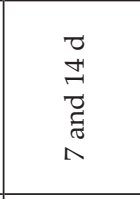 & 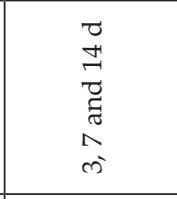 \\
\hline 品 & 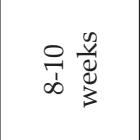 & 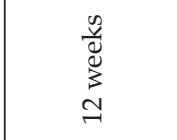 & 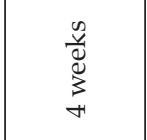 & 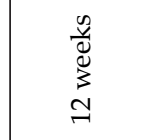 & 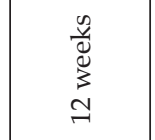 & 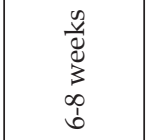 & 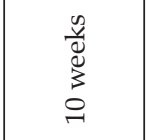 & 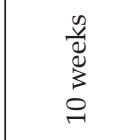 & 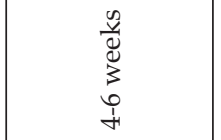 & 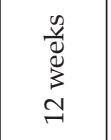 & 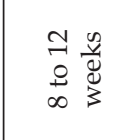 & $\begin{array}{l}\frac{n}{0} \\
\vdots \\
\vdots \\
\infty \\
\infty\end{array}$ \\
\hline 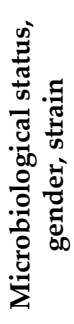 & 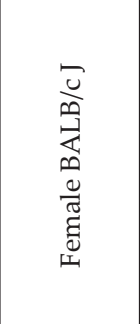 & 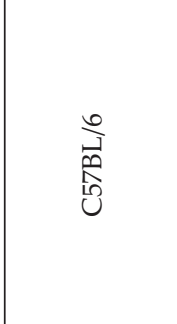 & 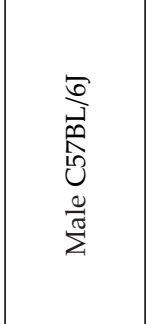 & 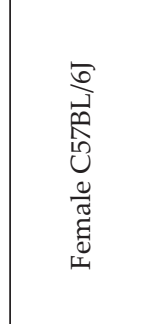 & $\begin{array}{l}\frac{00}{50} \\
\frac{1}{0} \\
\stackrel{0}{0} \\
0 \\
0 \\
0 \\
\frac{0}{\pi} \\
\frac{\pi}{2}\end{array}$ & 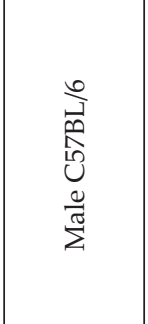 & 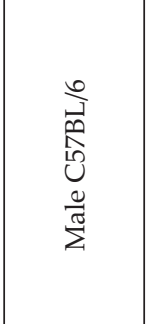 & $\begin{array}{l}\stackrel{0}{10} \\
0 \\
0 \\
0 \\
0 \\
\frac{0}{\tilde{0}} \\
\Sigma\end{array}$ & 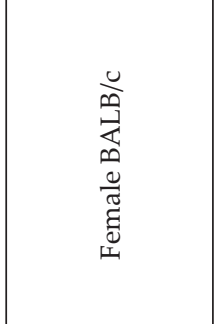 & 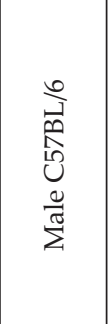 & 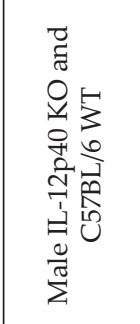 & 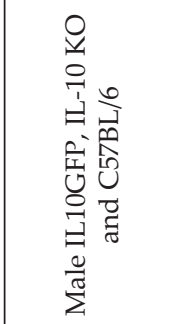 \\
\hline 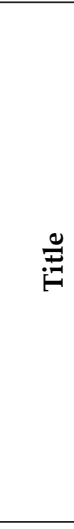 & 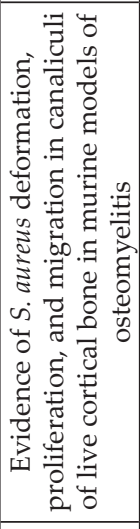 & 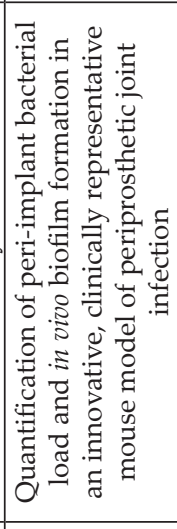 & 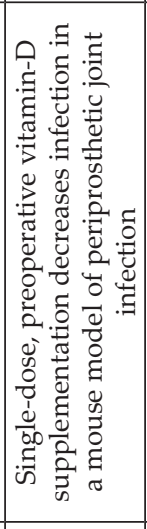 & 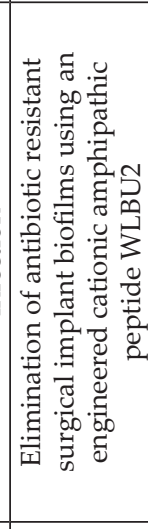 & 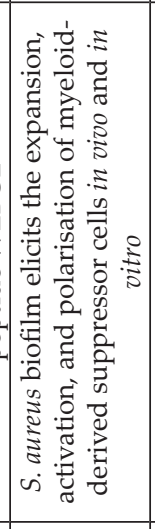 & 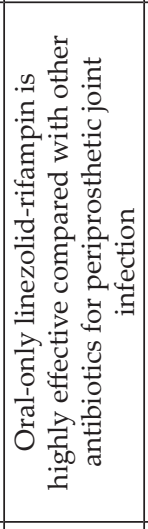 & 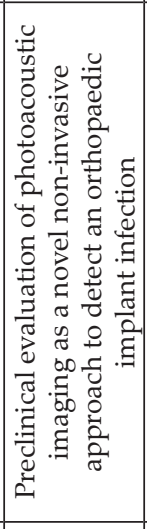 & 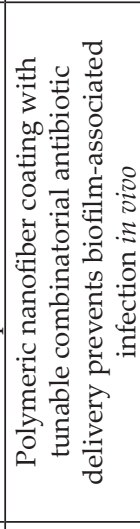 & 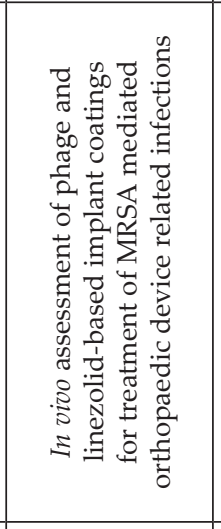 & 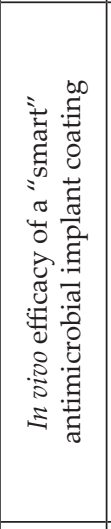 & 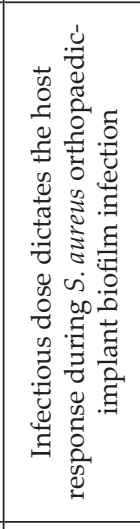 & 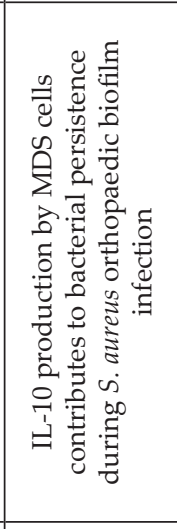 \\
\hline 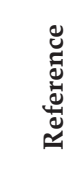 & 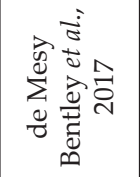 & 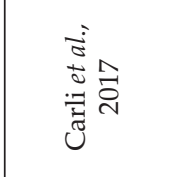 & 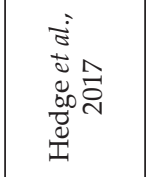 & 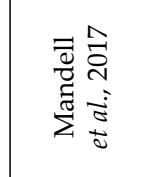 & 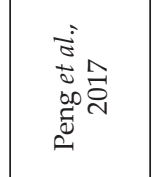 & 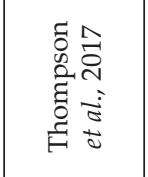 & 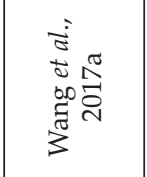 & 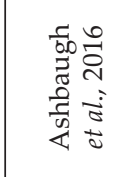 & 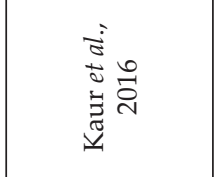 & 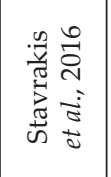 & 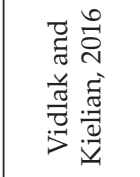 & 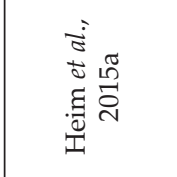 \\
\hline
\end{tabular}




\begin{tabular}{|c|c|c|c|c|c|c|c|c|c|c|c|}
\hline 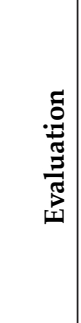 & 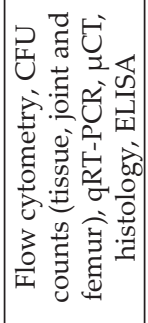 & 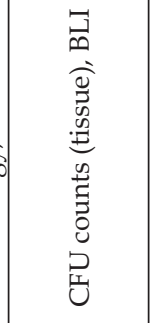 & 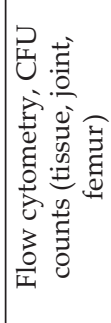 & $\begin{array}{l}\vec{J} \\
\dot{\infty} \\
\underline{z}\end{array}$ & 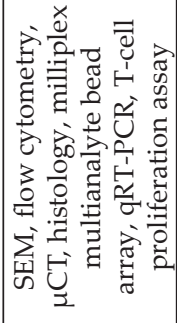 & 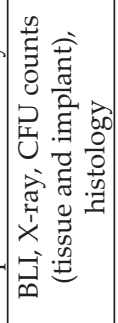 & 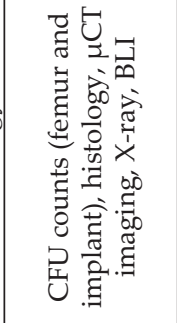 & 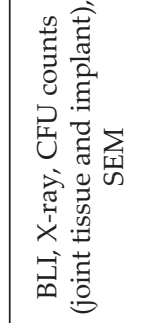 & 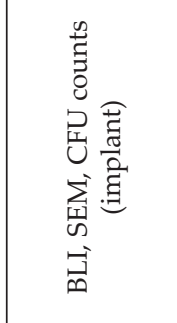 & 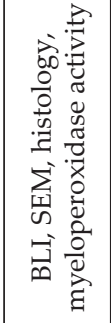 & 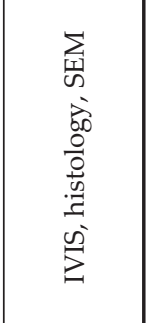 \\
\hline 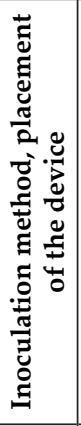 & 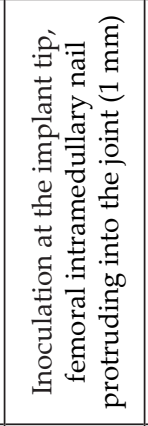 & 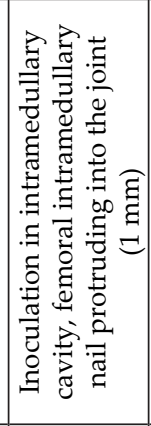 & 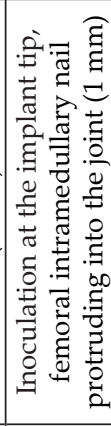 & 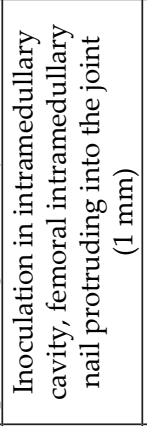 & 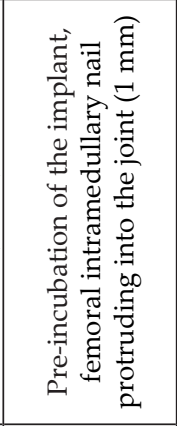 & 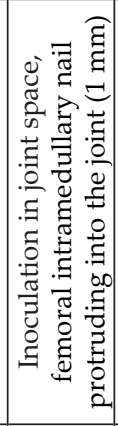 & 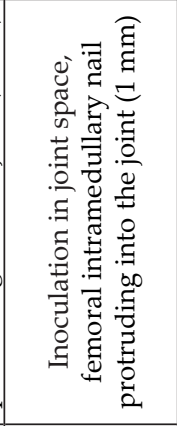 & 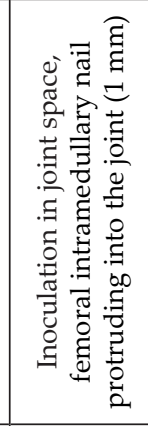 & 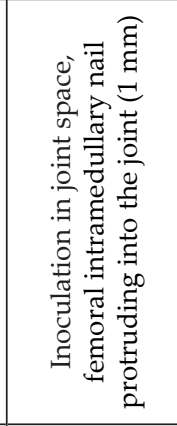 & 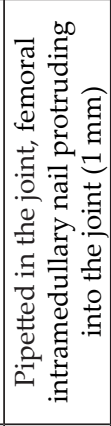 & 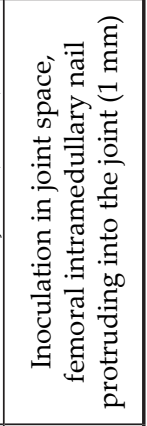 \\
\hline 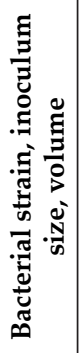 & 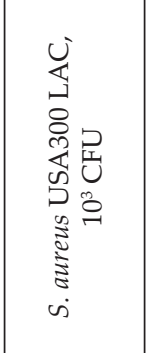 & 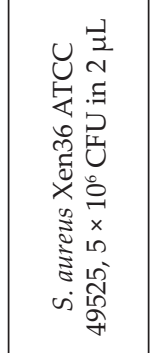 & 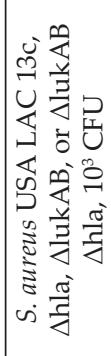 & 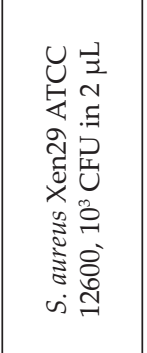 & 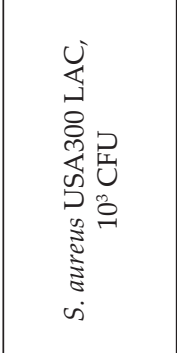 & 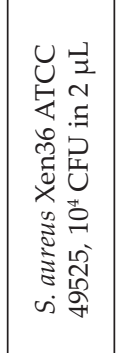 & 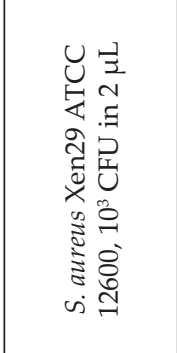 & 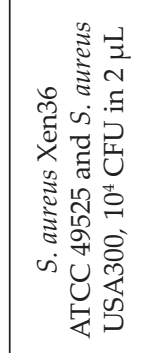 & 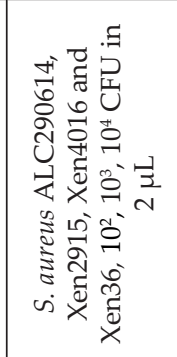 & 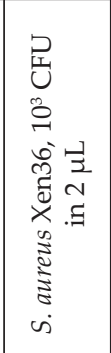 & 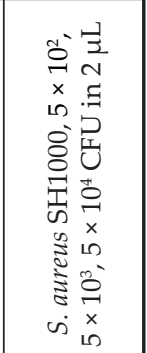 \\
\hline 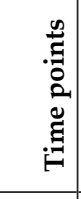 & 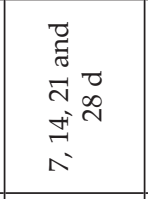 & 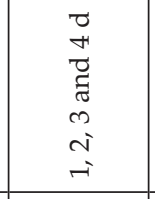 & $\begin{array}{l}\tilde{D} \\
\Lambda \\
\tilde{J} \\
\tilde{\sigma} \\
\tilde{n}\end{array}$ & 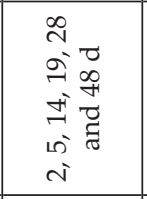 & 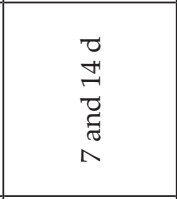 & 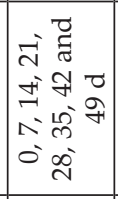 & 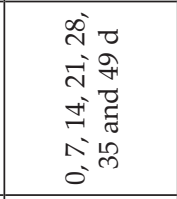 & $\begin{array}{l}\tilde{D} \\
\Lambda \\
\tilde{D} \\
\tilde{\sigma} \\
0 \\
0 \\
0\end{array}$ & 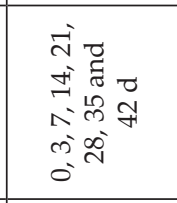 & 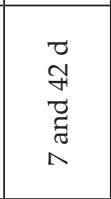 & 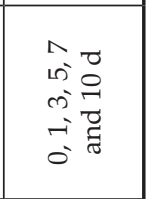 \\
\hline$\underset{\alpha}{80}$ & 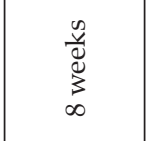 & $\begin{array}{l}\frac{n}{0} \\
\frac{\pi}{2} \\
3 \\
\vdots \\
\vdots\end{array}$ & $\begin{array}{l}\frac{v}{v} \\
0 \\
\vdots \\
\infty\end{array}$ & 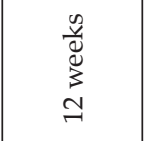 & $\begin{array}{l}\tilde{v} \\
\frac{\tilde{g}}{0} \\
\sum_{\infty}\end{array}$ & $\begin{array}{l}\stackrel{n}{v} \\
\vdots \\
\vdots \\
\infty \\
\infty\end{array}$ & 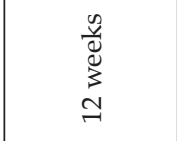 & 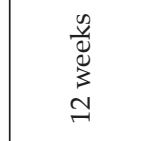 & 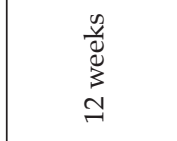 & 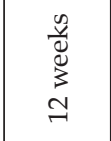 & 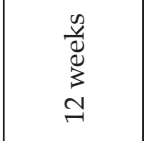 \\
\hline 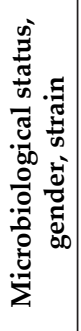 & 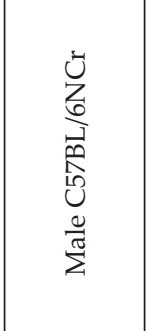 & 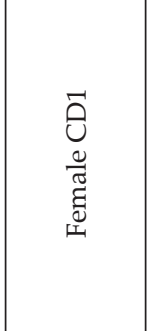 & 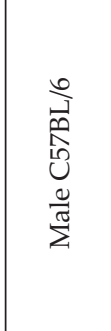 & 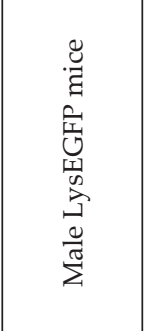 & 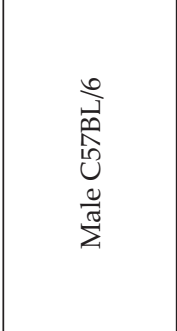 & 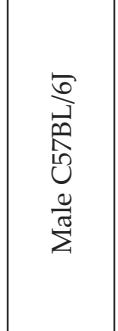 & 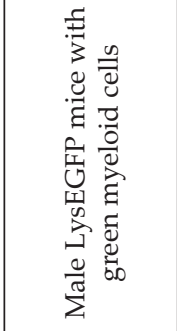 & 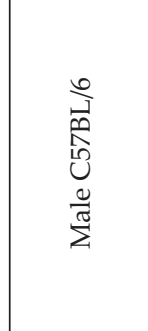 & 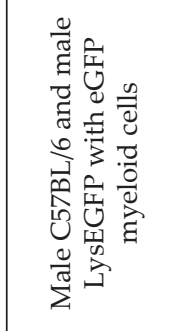 & 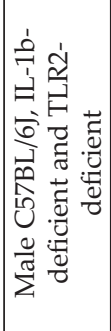 & 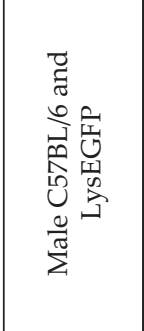 \\
\hline$\stackrel{\varrho}{\Xi}$ & 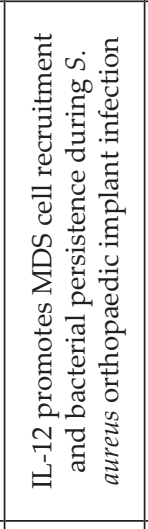 & 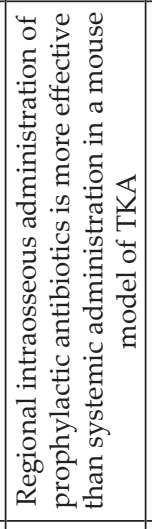 & 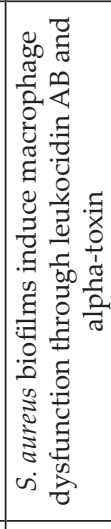 & 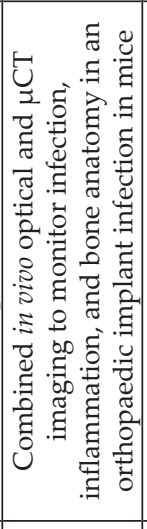 & 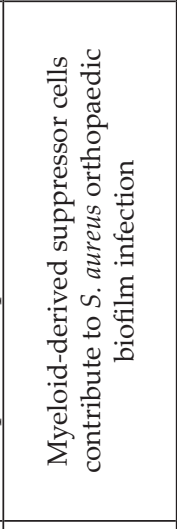 & 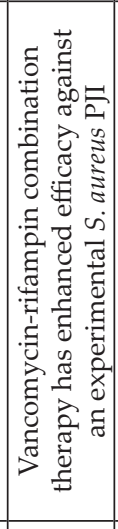 & 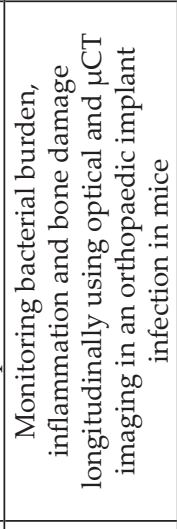 & 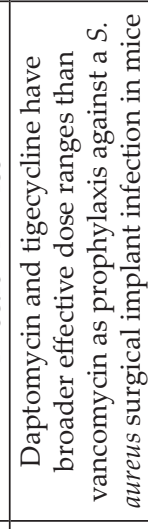 & 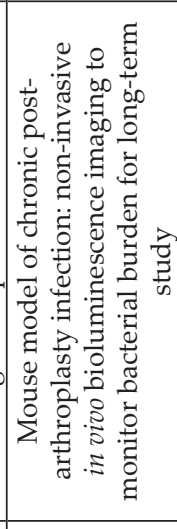 & 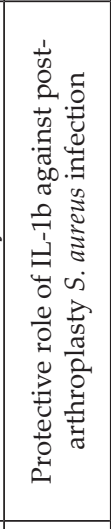 & 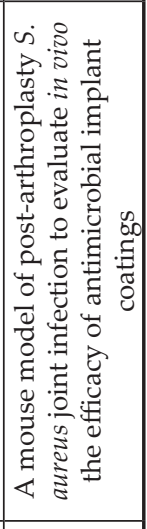 \\
\hline 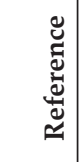 & 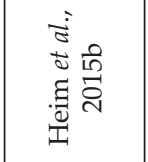 & 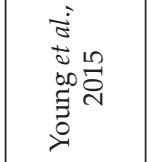 & 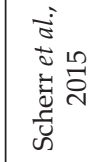 & 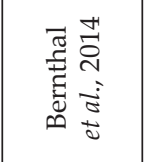 & 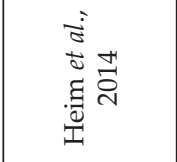 & 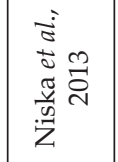 & 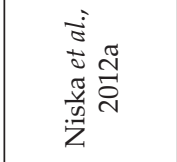 & 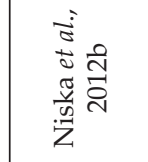 & 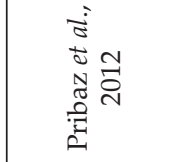 & 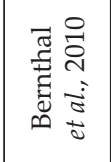 & 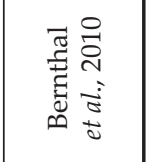 \\
\hline
\end{tabular}




\begin{tabular}{|c|c|c|c|c|c|c|c|c|}
\hline 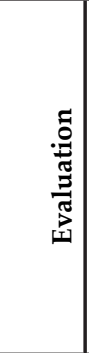 & 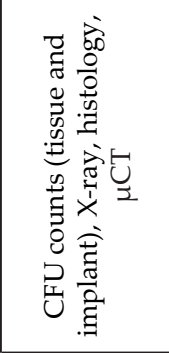 & 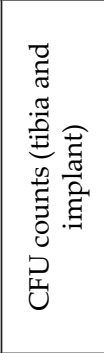 & 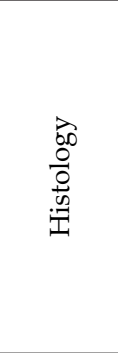 & 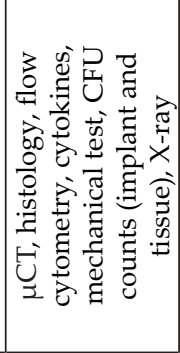 & 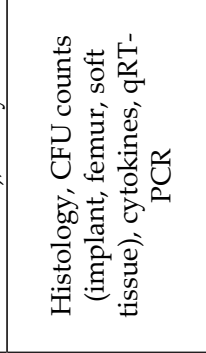 & 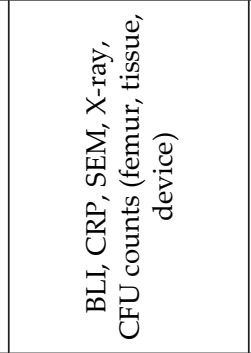 & 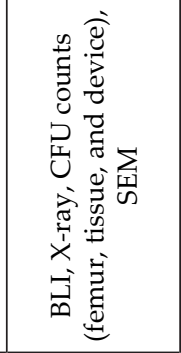 & 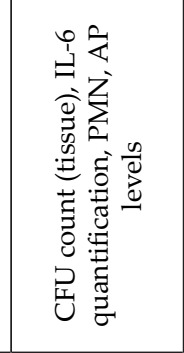 \\
\hline 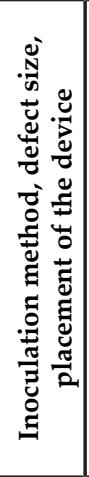 & 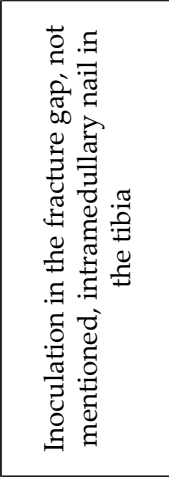 & 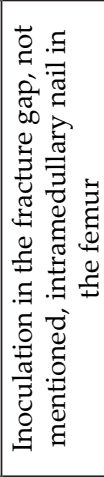 & 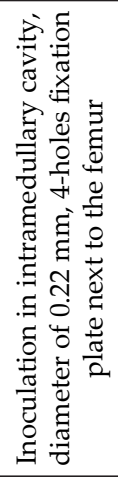 & 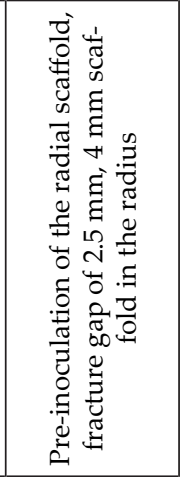 & 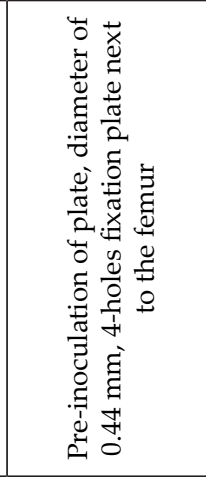 & 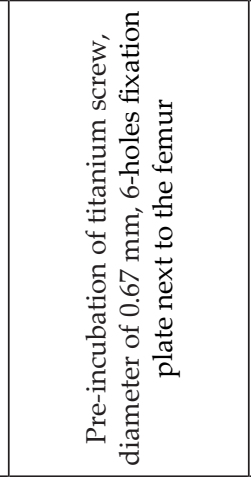 & 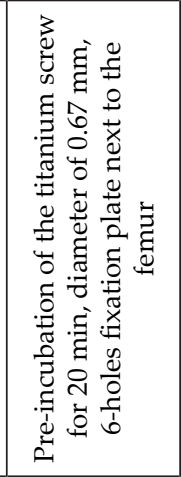 & 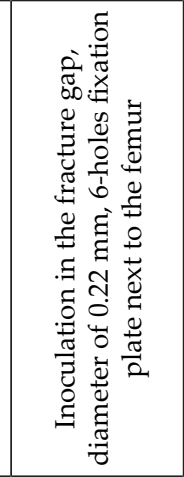 \\
\hline 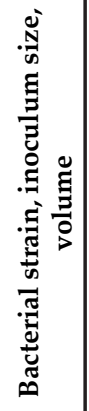 & 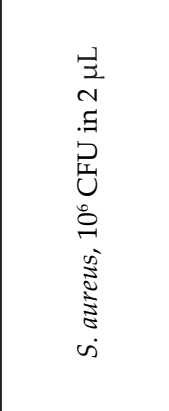 & 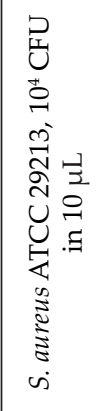 & 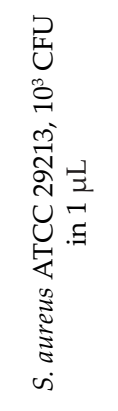 & 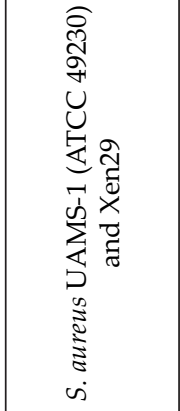 & 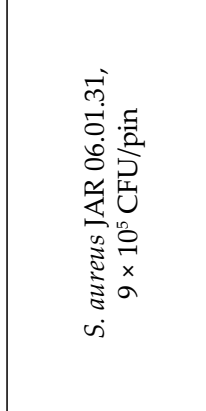 & 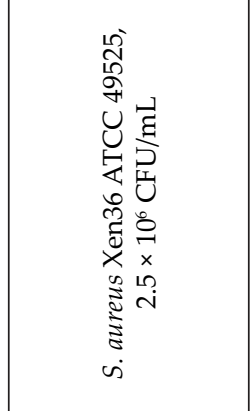 & 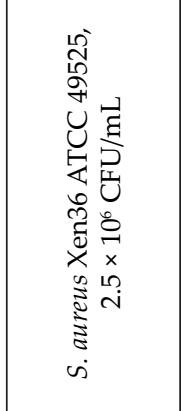 & 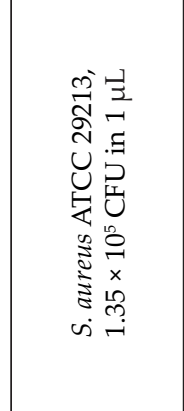 \\
\hline 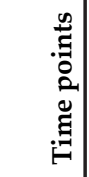 & 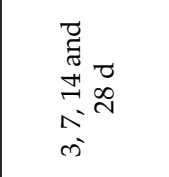 & $\begin{array}{l}\vec{J} \\
\vec{J}\end{array}$ & 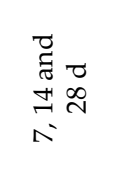 & & $\begin{array}{l}\tilde{D} \\
\hat{N} \\
\tilde{\sigma} \\
0 \\
0 \\
-\end{array}$ & 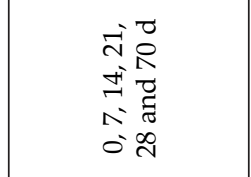 & 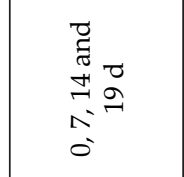 & $\begin{array}{l}\overrightarrow{7} \\
\mathbb{7} \\
\vec{\Xi} \\
\tilde{D}\end{array}$ \\
\hline$\stackrel{8}{80}$ & 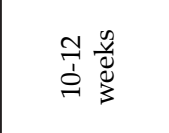 & 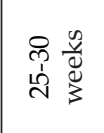 & 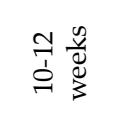 & 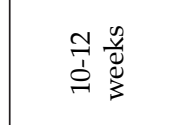 & 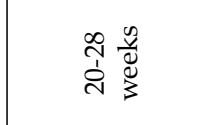 & 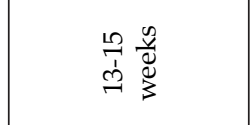 & 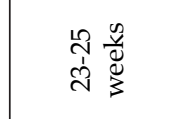 & 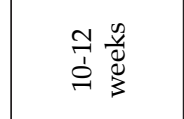 \\
\hline 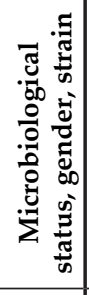 & $\begin{array}{l}\stackrel{0}{10} \\
0 \\
0 \\
0 \\
0 \\
\frac{0}{\pi} \\
\Sigma\end{array}$ & 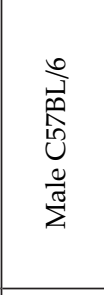 & 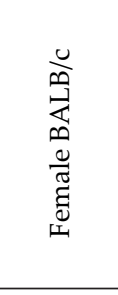 & 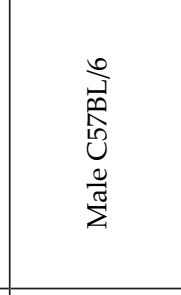 & 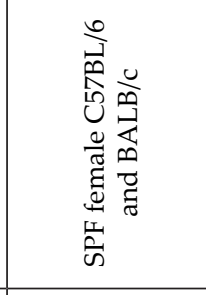 & 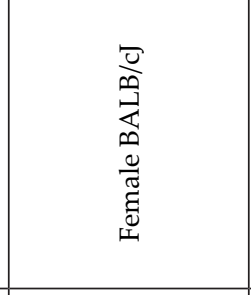 & 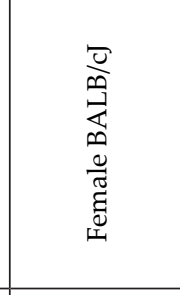 & 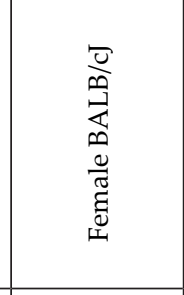 \\
\hline 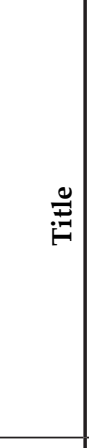 & 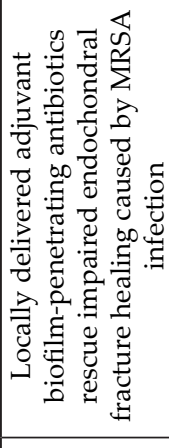 & 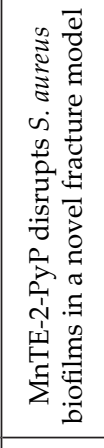 & 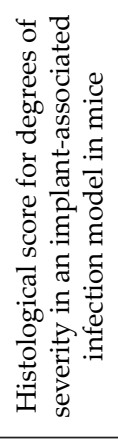 & 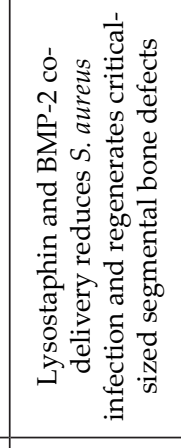 & 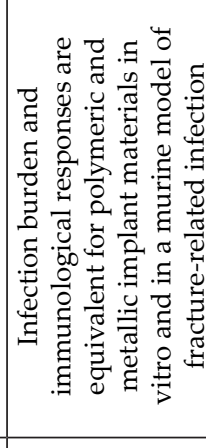 & 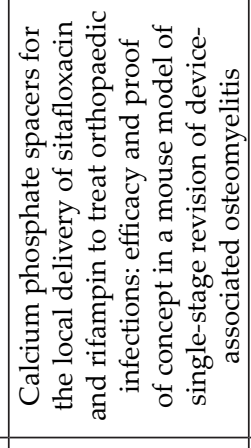 & 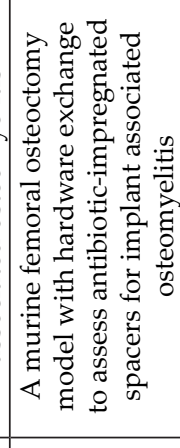 & 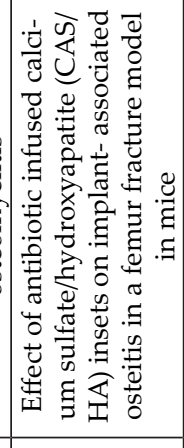 \\
\hline 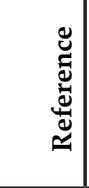 & 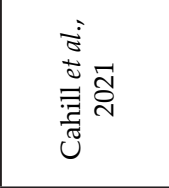 & 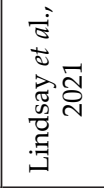 & 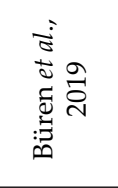 & 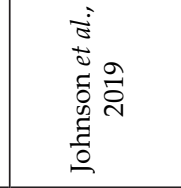 & 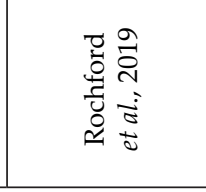 & 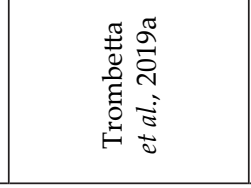 & 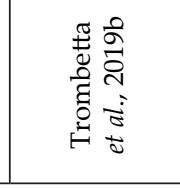 & 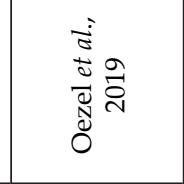 \\
\hline
\end{tabular}




\begin{tabular}{|c|c|c|c|c|c|c|c|c|c|}
\hline 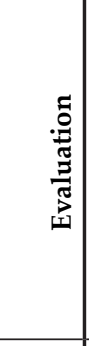 & 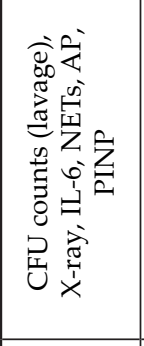 & 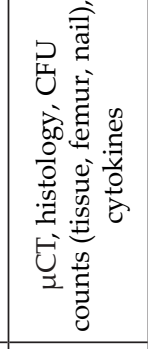 & 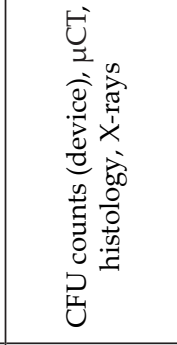 & 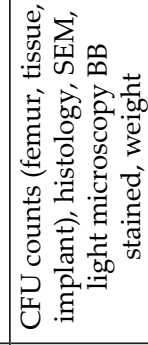 & 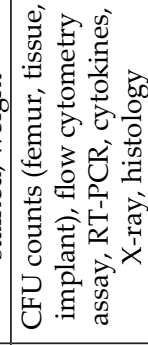 & 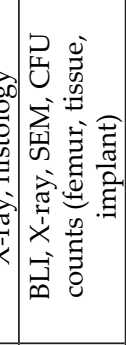 & 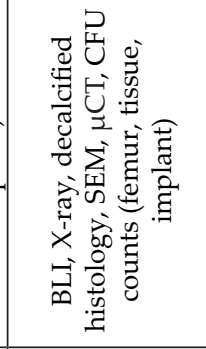 & 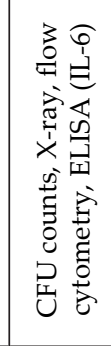 & 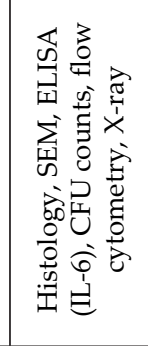 \\
\hline 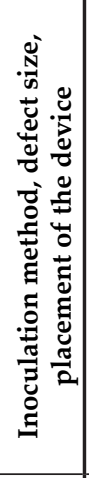 & 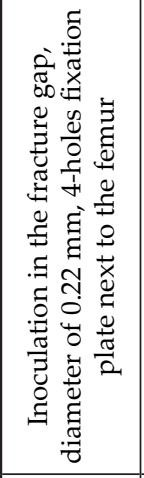 & 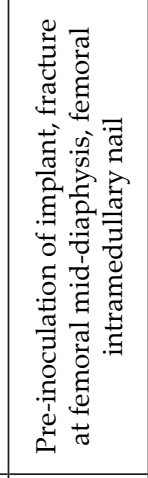 & 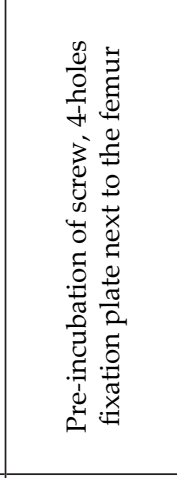 & 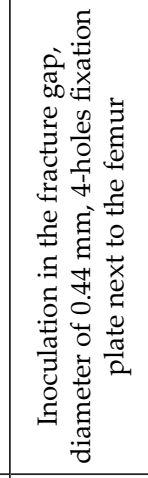 & 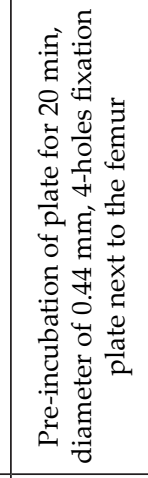 & 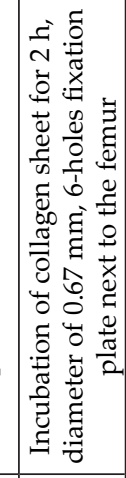 & 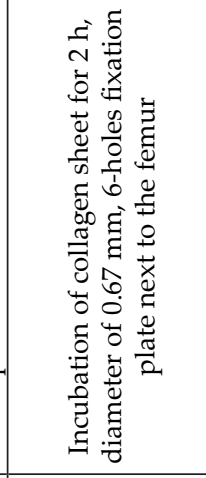 & 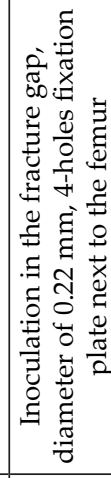 & 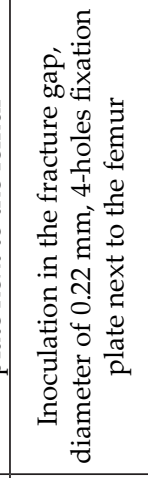 \\
\hline 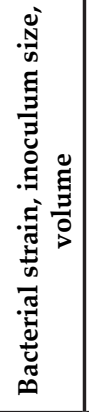 & 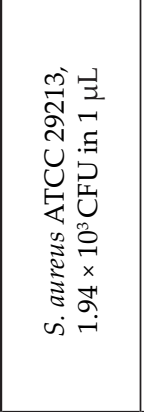 & 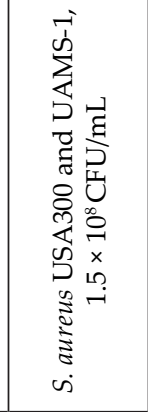 & 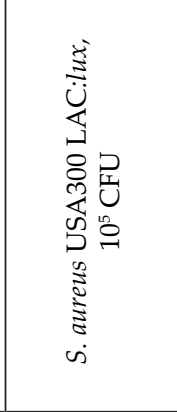 & 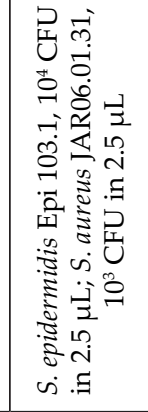 & 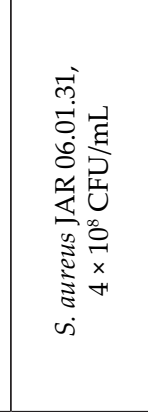 & 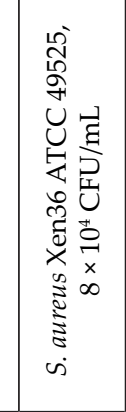 & 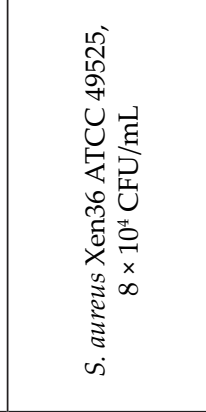 & 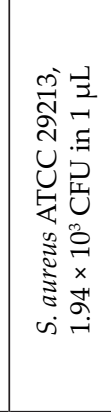 & 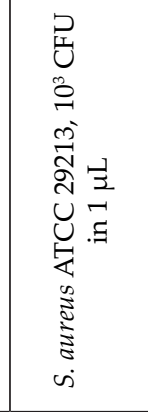 \\
\hline 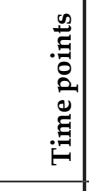 & 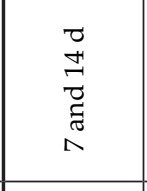 & $\Sigma_{\Lambda}$ & 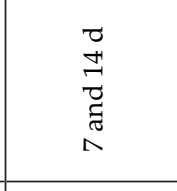 & 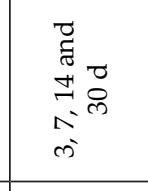 & 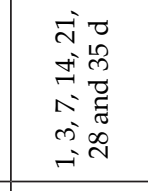 & 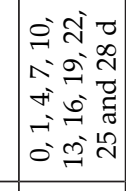 & 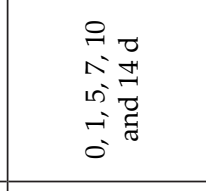 & 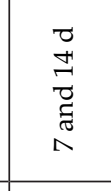 & 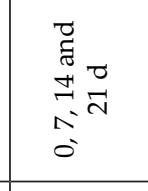 \\
\hline 总 & 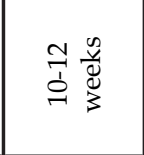 & 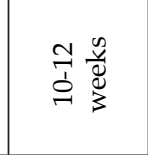 & 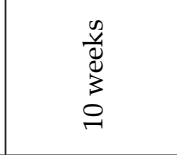 & 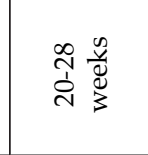 & 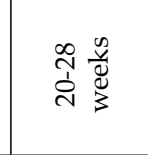 & 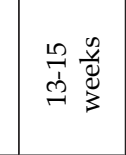 & 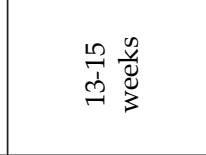 & 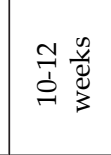 & 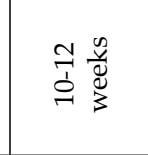 \\
\hline 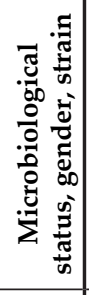 & 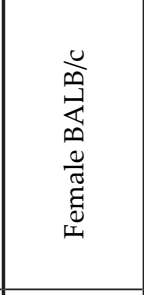 & 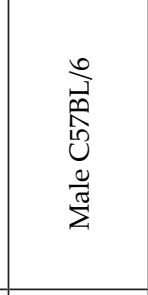 & 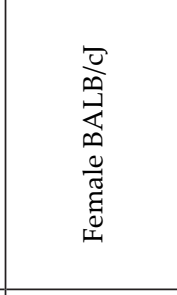 & 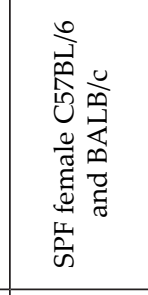 & 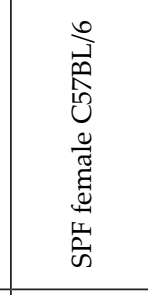 & 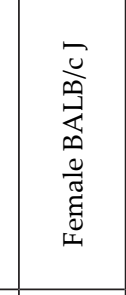 & 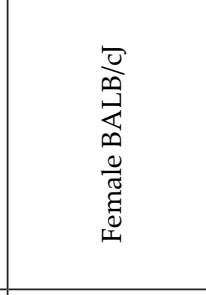 & 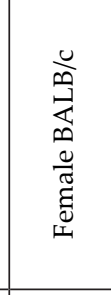 & 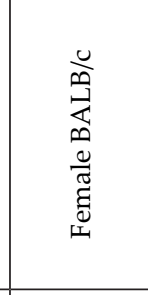 \\
\hline$\stackrel{0}{\Xi}$ & 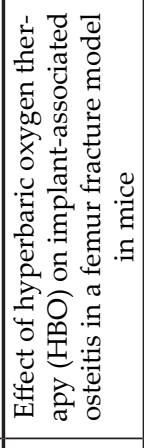 & 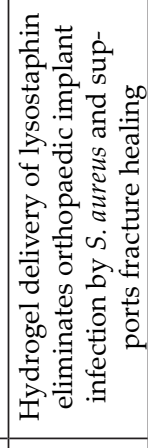 & 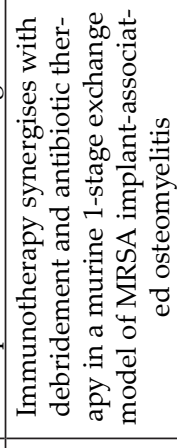 & 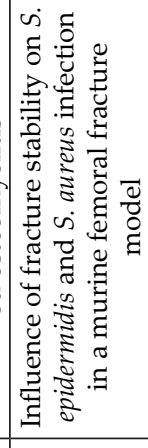 & 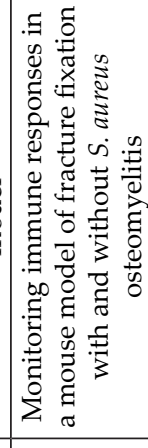 & 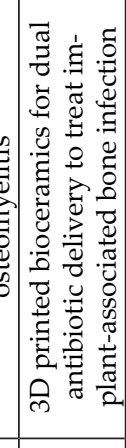 & 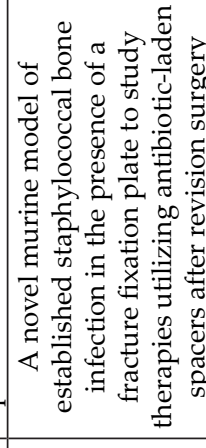 & 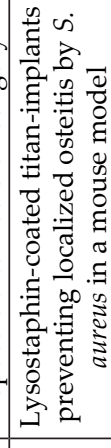 & 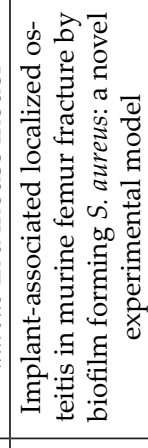 \\
\hline 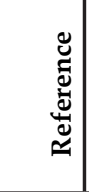 & 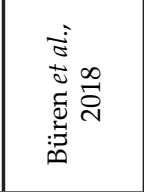 & 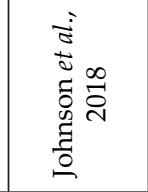 & 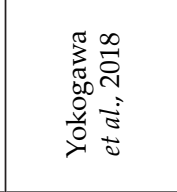 & 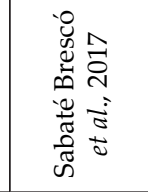 & 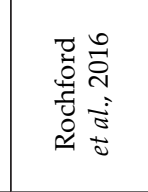 & 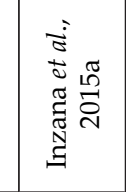 & 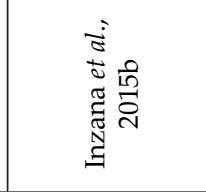 & 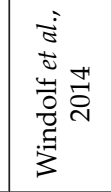 & 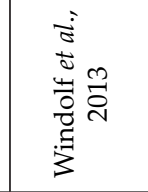 \\
\hline
\end{tabular}


unicortical defect are also included. Tables 3, 4 and 5 also list the type of device and placement of the device. In Table 5, the type of diabetes induced is also included. Table 6 also includes the size of the fracture, type of device and placement of the device.

\section{Analysis of the data}

\section{Characteristics of the mouse}

The studies retrieved in the systematic search have used mice with different characteristics such as inbred strain, age and gender. Differences in mouse strain, age, gender, microbiome composition and the use of conventional or SPF animals could influence the osteomyelitis and fracture healing process. Therefore, it is important to take these characteristics into account to properly choose the animal model.

Inbred mouse strains harbour differences in bone density and bone length. Bone composition differs among inbred mouse strains. For instance, the femur density of C57BL/6J mice $(0.45 \mathrm{mg} /$ $\left.\mathrm{mm}^{3}\right)$ is significantly lower than that of $\mathrm{C} 3 \mathrm{H} / \mathrm{HeJ}$ mice $\left(0.69 \mathrm{mg} / \mathrm{mm}^{3}\right)$ and of BALB/c mice $(0.55 \mathrm{mg} /$ $\mathrm{mm}^{3}$ ). Interestingly, the femur of $\mathrm{C} 3 \mathrm{H} / \mathrm{HeJ}$ mice is significantly shorter than that of BALB/C and C57BL/6]

a

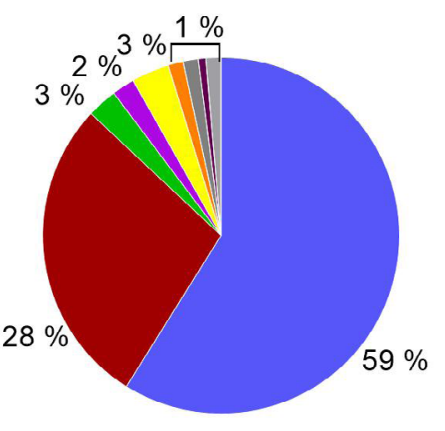

Total $=146$

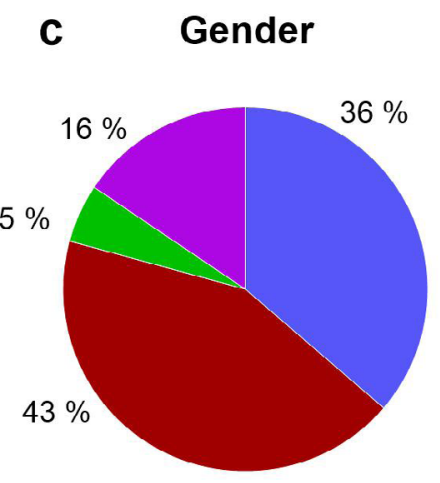

Total $=135$ mice at all ages measured (Beamer et al., 1996; Kohler et al., 2005). Most researchers selected C57BL/6 (59\%) or BALB/c (28\%) mice for osteomyelitis studies (Fig. 3a). Regarding infection development, in a fracturerelated infection study using $S$. epidermidis, BALB/C mice showed larger bacterial counts and formation of larger abscesses compared to C57BL/6 mice (Sabaté Brescó et al., 2017). In cases of infection with $S$. aureus, opposite results were observed regarding the ability of C57BL/6 and BALB/c mice to clear the infection in a bone-implant-related infection study (Nishitani et al., 2015b; Prabhakara et al., 2011b). The authors observed that $58 \%, 50 \%$ and $75 \%$ of the BALB/c mice cleared the infection at 21, 28 and $49 \mathrm{~d}$ post-infection, respectively; while $100 \%$ of the C57BL/6 mice were unable to clear the infection even 49 d post-infection (Prabhakara et al., 2011b). Moreover, a study comparing $S$. aureus infection, using a bioluminescent $S$. aureus strain, in C57BL/6 and BALB/c mice only showed a brief peak of high intensity bioluminescence at $3 \mathrm{~d}$ in the BALB/c strain, characterising active infection, while the C57BL/6 showed high bioluminescence intensity that extended from day 3 through day 7 (Nishitani et al., 2015b). The immune system of C57BL/6 and BALB/c mice differs (Sellers et al., 2012), with C57BL/6 mice being more differentiated towards a Th1-like phenotype
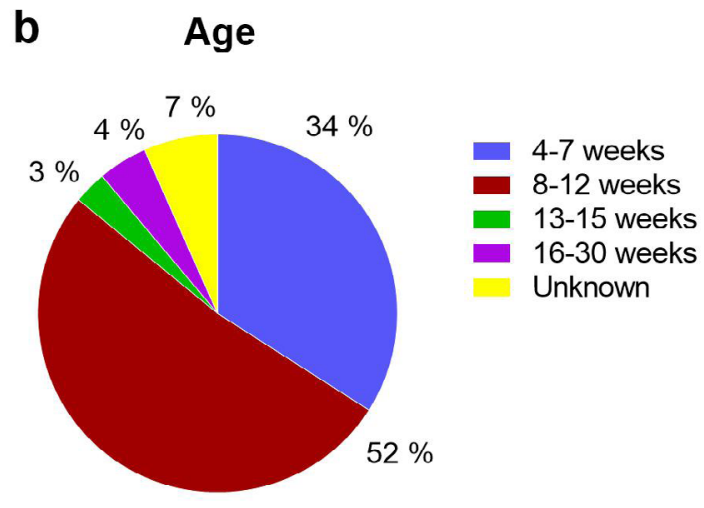

Total $=135$

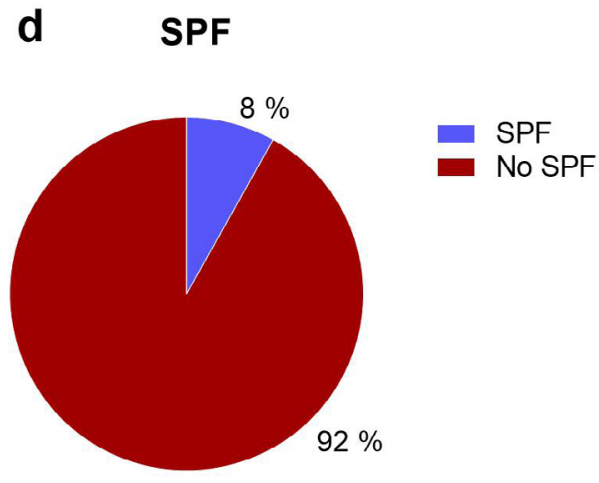

Total $\mathbf{=} 135$

Fig. 3. Classification of the osteomyelitis mouse models by (a) mouse strain, (b) age, (c) gender and (d) SPF. 135 studies were included in the graphs. In panel a, some studies included more than one inbred strain. Therefore, there is a total number of 146 studies. 
and BALB/c mice towards a Th2-like phenotype in the presence of an S. aureus infection (Prabhakara et al., 2011b). This needs to be further investigated to understand the response in each mouse strain and allow the researcher to choose the proper mouse strain for a study.

The animal ages determines to the quality of their bones and the time required for complete healing of a fracture (Ferguson et al., 2003; Haffner-Luntzer et al., 2016). A faster healing process will reduce the risk and period of the infection. Mice are sexually mature at 6-8 weeks of age (Jilka, 2013). Therefore, mice of this age (34 \%) or slightly older (9-12 weeks, $52 \%$ ) are often selected for osteomyelitis studies because their bones are no longer growing (Fig. 3b). Some authors emphasise the importance of using older mice, 16-30 weeks old, when the animals reach skeletal maturity (Rochford et al., 2016; Sabaté Brescó et al., 2017); however, those are less often used (7\%). The skeletal maturity is characterised by the larger size and greater strength of the bones but also by the degree of mineralisation (Ferguson et al., 2003). The peak of mineralisation is not achieved until an age of 20 weeks in C57BL/6 mice (Brodt et al., 1999; Ferguson et al., 2003).

The animal gender may also influence the healing of the bone and development of infection. For example, hormonal cycles in the females can have a significant influence on bone repair. On the other hand, males are more territorial and may require separate cages to avoid fighting, making them more labour-intensive and expensive to keep (Mills and Simpson, 2012). Of the osteomyelitis in vivo studies collected in the present review, $36 \%$ used male mice, $43 \%$ used female mice, $5 \%$ used both genders and $16 \%$ did not mention the gender (Fig. 3c).

The gut microbiome is composed of a wide range of species of microorganisms, including bacteria, yeast and viruses, and provides essential health benefits to its host, particularly by regulating the immune homeostasis (Vlasova et al., 2019). A recent study has revealed that changes in the gut microbiome can influence the development of bone infections in mice (Hernandez et al., 2019). Mice with a disrupted microbiome are more likely to develop a bone infection than mice with a non-modified microbiome; moreover, mice with a disrupted microbiome also show a reduced immune response to the infection (Hernandez et al., 2019). However, more research is necessary to better understand how the gut microbiome influences the response to the infection and help researchers to decide on the optimal features of the mouse strain to select for an in vivo study (Hernandez et al., 2019). Moreover, to reduce the presence of unwanted infections affecting the experiments, some researchers prefer to use SPF animals. The use of SPF animals has the additional benefits of being cost-saving as it minimises the number of animals used for a study (Letson et al., 2019). However, SPF animals may have undesired changes in their immune system that may be linked to modifications in their gut microbiome (Letson et al., 2019). $8 \%$ of the studies analysed in the present review chose SPF mice for their in vivo osteomyelitis studies (Funao et al., 2016; Horst et al., 2012; Isogai et al., 2020; Rochford et al., 2016; Sabaté Brescó et al., 2017; Szafranska et al., 2014) but in $92 \%$ of studies conventional mice were used (Fig. 3d).

\section{Bacterial species related to osteomyelitis}

$S$. aureus has been the main bacterial species chosen ( $90 \%$ ) for in vivo experiments because it is the most common pathogen causing osteomyelitis (Kavanagh et al., 2018; Fig. 4a). Its ability to cause osteomyelitis is related to the presence of features, known as virulence factors, that allow the bacteria to attach to bones and foreign bodies and form biofilms, to evade the immune system and to cause harmful toxic effects. These virulence factors can be classified by their function as adherence factors, immunomodulatory factors, enzymes and toxins. In the present review, relevant virulence factors were identified and further described below.

Adherence virulence factors such as bacterial adhesins or coagulase can be crucial for bacterial attachment and establishment of an infection. Bacterial adhesins are proteins present on the bacterial surface that can interact with and bind to eukaryotic extracellular (matrix) proteins. Among these adhesins, Cna, FnbA and FnbB are relevant for tissue colonisation and development of bone infections. FnbA and FnbB are present in $98 \%$ and $99 \%$ of clinical isolates from osteomyelitis, respectively (Arciola et al., 2005a). An in vivo mouse study has shown the essential role of $\mathrm{Cna}$ in the bone colonisation by comparing the infectivity of the $S$. aureus UAMS-1 (ATCC 49230) strain expressing Cna and FnbA and its isogenic Cna mutant known as S. aureus UAMS-237 (Elasri et al., 2002). The strain UAMS-1 is an isolate derived from the clinical strain USA200 and is one of the most widely used S. aureus strains in osteomyelitis research (Li et al., 2008). Only the Cna positive bacterial strain was able to colonise the murine tibia (Elasri et al., 2002). Thus, to be able to establish an infection in the bone it is necessary to use a Cna positive strain.

Immune evasion is also an important virulence factor for bacteria to establish and maintain the infection in the bone. S. aureus has developed strategies to persist and evade clearance by the immune system and establish a chronic bone infection (Tuchscherr et al., 2017). For example, SigB is an important transcription factor contributing to the avoidance of bacterial clearance by the immune response (Tuchscherr et al., 2017). S. aureus lacking SigB induces large numbers of small abscesses, which contributes to the elimination of the bacteria in murine osteomyelitis (Tuchscherr et al., 2017). The study also showed that a deletion of $\operatorname{sig} B$ results in a reduced production of SarA (Tuchscherr et al., 
2017). SarA plays a key role in the establishment of the osteomyelitis in a haematogenous murine model (Blevins et al., 2003). A deletion of $\operatorname{sar} A$ in $S$. aureus UAMS-1 and $S$. aureus RN6390 results in a significantly reduced number of CFU compared to the wild-type strains (Blevins et al., 2003).

The extent of bone destruction caused by the infection is also related to virulence factors such as toxins, e.g. PSMs. PSMs are amphipathic peptides with functions as cytotoxins and pro-inflammatory inducers (Cassat et al., 2013). The role of PSMs was studied in an osteomyelitis mouse model indicating that PSMs cause osteoblast cell death and contribute to the destruction of the bone (Cassat et al., 2013; Loughran et al., 2016).

Although antimicrobial resistance is not a virulence factor, in certain situations it is a key factor in the development of an infection and it may be considered to be a virulence-like factor. Antimicrobial resistance plays a key role in persistent osteomyelitis due to the fast development of resistance to multiple antibiotics and the difficulty in treating these infections, causing a persistent osteomyelitis. MRSA is responsible for $40-60 \%$ of all S. aureus infections. Therefore, several authors chose MRSA strains for the osteomyelitis mouse model (Jørgensen et al., 2014; Loughran et al., 2016). In addition, it is important to consider the antimicrobial resistance profile of the bacterial strain when testing antimicrobial strategies. Nevertheless, the resistant strain needs to be virulent

a

Species

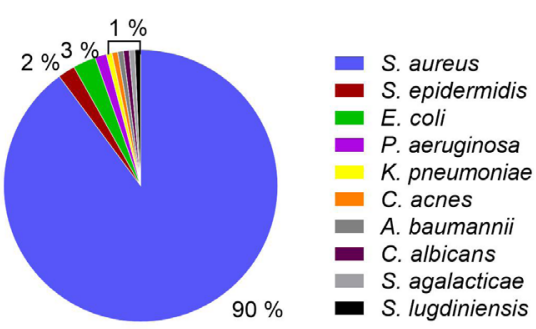

Total $=148$

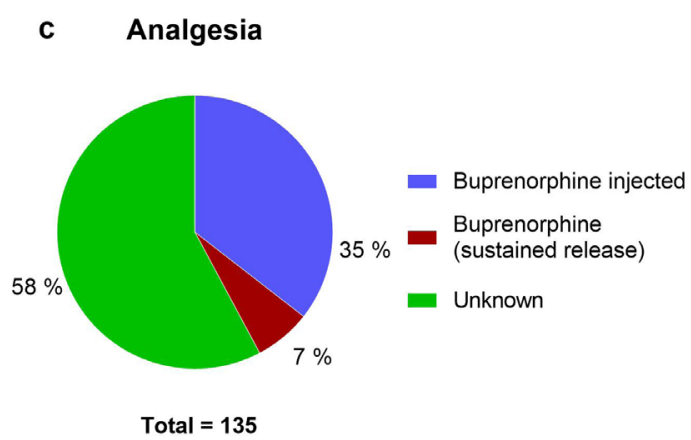

enough, with the presence of virulence factors such as adhesins, to establish an infection in the first place.

$90 \%$ of the mouse osteomyelitis studies included in the present review were focused on $S$. aureus infections (Fig. 4a). However, other bacterial pathogens can cause osteomyelitis and can also be used to establish an infection and further understand the pathophysiology of the disease. For example, Sabaté Brescó et al. (2017) compared the development of fracture-related infection caused by $S$. aureus JAR 06.01.31 and S. epidermidis 103.1. Compared to S. aureus, S. epidermidis infection was less intense, leading to fewer bacteria, causing less bone damage and affecting less the animals' weight. Tomizawa et al. (2020) compared the pathogenic potential of an S. epidermidis strain RP62a versus $S$. aureus strain USA300, both methicillin-resistant strains, in a boneimplant-related infection mouse model. As expected, $S$. aureus caused a more aggressive and damaging osteomyelitis than S. epidermidis. Nevertheless, S. epidermidis formed a large biofilm on the implant and caused less inflammation than $S$. aureus.

Shandley et al. (2012) aimed to establish a boneimplant-related infection in mice with $S$. aureus, $P$. aeruginosa and $K$. pneumoniae to evaluate the antimicrobial activity of hyperbaric oxygen therapy. Their results showed that $P$. aeruginosa and $S$. aureus are equally capable of establishing the infection in the bone. However, K. pneumoniae was not able to infect the bone well and was cleared before infection

\section{b Anaesthesia}

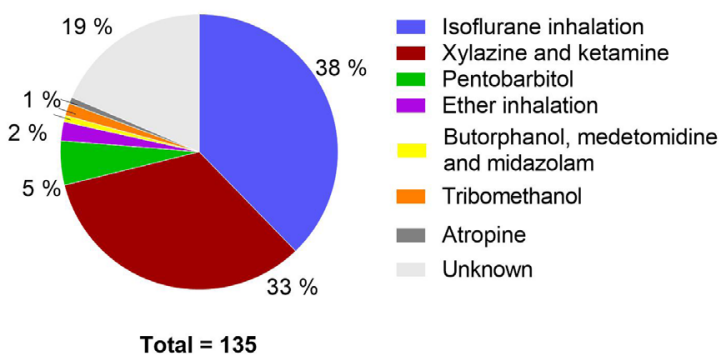

d Imaging technique

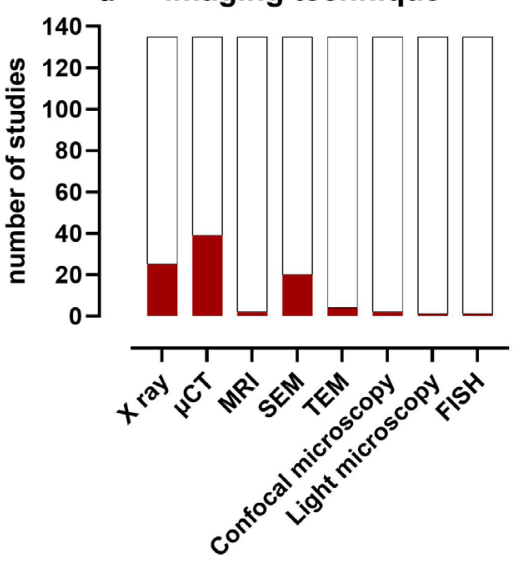

Fig. 4. Classification of the osteomyelitis mouse models by (a) bacterial or fungal pathogen used to infect the animals, (b) image technique used to assess the infection, (c) anaesthesia used to perform the surgery and (d) analgesia used after the surgery. 135 studies were included in the graphs. 
was established. Boles et al. (2018) established a polymicrobial infection with $S$. aureus UAMS- 1 and $E$. coli ATCC 25922 in a mouse implant infection model. E. coli was not found either in bones or on implants after $7 \mathrm{~d}$.

Crane et al. (2009) studied the efficacy of colistin beads in an implant-related osteomyelitis mouse model infected with $S$. aureus or A. baumannii clinical isolates. In contrast to the osteolytic response in the presence of $S$. aureus, A. baumannii caused an osteoblastic bone formation response around the infected devices. S. aureus was also associated with the presence of biofilm in necrotic bone sections, whereas no biofilm was found in the necrotic tissue in the animals infected with A. baumannii (Crane et al., 2009). Shiono et al. (2016) studied the development of osteomyelitis by $C$. acnes with and without a titanium bone implant. $C$. acnes survived attached to the implant for 6 months, causing a delayed infection, while it only survived $28 \mathrm{~d}$ in the control group without an implant (Shiono et al., 2016). Masters a et al. (2021) studied the pathogenesis of S. agalacticae versus $S$. aureus in a bone implant infection model. Their results showed that $S$. agalacticae colonies were only retrieved from the soft tissue and bone but not from the implant after $14 \mathrm{~d}$ of infection. S. agalacticae caused less osteolysis and no loss of weight compared to $S$. aureus. Moreover, their TEM images revealed the vasculotropic pathogenesis of $S$. agalacticae compared to the osteotropic behaviour of S. aureus (Masters et al., 2021).

To summarise, it is advisable to consider all of these bacterial features (e.g. bacterial species, virulence factors and antimicrobial resistance profile) when choosing the appropriate bacterial strain for the intended in vivo osteomyelitis mouse studies. In addition, the use is recommended of a strain with a well-documented origin, phenotypic and genotypic profile (and to ensure availability of the strain in view of repeatability and reproducibility of the experiments), proved pathogenicity of the strain and strain characteristics (e.g. biofilm formation, panel of adhesins, bacterial toxins and antimicrobial resistance) (Moriarty et al., 2019).

\section{Inoculation method and dose}

Depending on the research question, the bacterial inoculum can be administered in different ways. To mimic a contamination during trauma or surgery, the inoculum can be administered during implantation or defect/fracture formation by pipetting it onto the wound site or applying it using an injector along or in the vicinity of the implant, bone defect or fracture (Thompson et al., 2017). It is advisable to use a low volume for the inoculum to prevent dispersion of the inoculum to other tissues. Therefore, 56 of the studies analysed in the present review used a small inoculum between 1 and $5 \mu \mathrm{L}$ (Tables 2-6).

To mimic a device contamination, the devices are pre-inoculated with the bacterial strain overnight or briefly before surgery (de Mesy Bentley et al., 2017;
Ishikawa et al., 2017; Li et al., 2008; 2017; Pribaz et al., 2012; Thompson et al., 2017). This method can be applied to evaluate the antimicrobial efficacy of contact-killing or antifouling surfaces (Ishikawa et al., 2017). Moreover, the pre-inoculation of the device can be used to create established biofilms to study an advanced stage of the infection and to evaluate more challenging antimicrobial treatment regimes. Nevertheless, it is advisable to not use a pre-inoculation method to evaluate the antimicrobial activity of a drug delivery system because the device may release the drug before the surgical implantation and the bacteria may be killed prior to implantation.

The injury can also be contaminated from the insertion of a surgical material into the wound such as a pre-inoculated silk suture (Yoshii, 2002a; Yoshii et al., 2002b), pre-inoculated agarose beads (Marriott et al., 2004; Marriott et al., 2005) or a pre-inoculated collagen sheet (Inzana et al., 2015b).

To mimic a late infection or haematogenous infection, the inoculum is injected into the bloodstream at a later point, for example, via the lateral tail vein (Blevins et al., 2003; Chadha et al., 1999; Elasri et al., 2002; Horst et al., 2012; Szafranska et al., 2014; Tuchscherr et al., 2017; Yoon et al., 1999) or the retroorbital sinus (Potter et al., 2020; Wang et al., 2017c). In these models, a larger inoculum volume, between 100 and $1,000 \mu \mathrm{L}$, is used to allow the inoculum to distribute throughout the bloodstream thus mimicking the haematogenous model.

In general, to create an osteomyelitis mouse model, it is advisable to perform dose-determining studies to establish the dose required to initiate a bone infection. The data collected in the present review show that a certain range of both injection volumes and numbers of CFU are used to establish a bone infection (Tables 1-6).

\section{Anaesthesia and analgesia in osteomyelitis models} The discomfort of the animals must be maximally reduced for ethical reasons but also because of the potentially adverse influence on experimental outcomes (Moriarty et al., 2019). Mouse anaesthesia demands a thorough knowledge of the mouse physiology and of the pharmacology of the anaesthetics and analgesics in the animals. Depending on the objectives of the experimental procedure, anaesthetics and analgesics can be administered through injection or inhalation (Adams and Pacharinsak, 2015; Gargiulo et al., 2012). In this section, the anaesthetics and analgesics that have been used for these osteomyelitis mouse models will be discussed.

In $40 \%$ of the 135 studies considered in the present review, mice were anaesthetised by inhalation and in $60 \%$ by injection. Inhaled anaesthetics, such as isoflurane, require the use of nose-cone ventilation during surgery, limiting the possibility of adjusting the mouse position, which usually is necessary to create a bone defect or fracture. Inhalation anaesthesia provides greater safety for prolonged 
procedures than injectable anaesthetics to maintain the animals in an anaesthesia of sufficient depth. Moreover, inhaled anaesthetics provide more control on the dose administered to the animal and are associated with a quicker recovery compared to injectable anaesthetics (Buitrago et al., 2008). In the osteomyelitis mouse studies, $38 \%$ of the studies used isoflurane and only 2 studies (2\%) used ether as inhaled anaesthetic (Yoshii et al., 2002a; 2002b, Fig. 4b). Injectable anaesthetics permit an easier manipulation of the animals during the surgical procedure, where the animals can undergo various positional changes during the process of creation of bone defect or fracture, which may be required for the placement of a fixation device (nail or plate). However, for longer surgical procedures, the use of inhaled anaesthetics is recommended to avoid the animals awakening during surgery. The most common injectable anaesthetics used within the osteomyelitis mouse models were the combination of xylazine and ketamine (33\%), which are inexpensive, easy to administer and pose no health risks to the researchers (Fig. 4b). Moreover, this combination provides good immobilisation with, additionally, a certain degree of analgesia. Unfortunately, the injectable anaesthetics may present a higher risk of overdose and cardiovascular and respiratory depression that may lead to an increased animal mortality (Buitrago et al., 2008).

Regarding the analgesics, they are administered before and after the surgical procedure. $35 \%$ of the studies injected between 0.01 and $0.05 \mathrm{mg} / \mathrm{kg}$ of buprenorphine (Pribaz et al., 2012; Shandley et al., 2012). In several studies (7\%), a polymeric matrix with buprenorphine was inserted into the wound to provide a sustained release of the drug for $72 \mathrm{~h}$ to minimise any additional distress (Niska et al., 2012a; Stavrakis et al., 2016; Wang et al., 2017a; Fig. 4c). However, in most of the studies (58\%), the analgesic procedure used was not specified.

\section{Osteomyelitis evaluation assays}

The development of an infection in the bone and surrounding tissue and its effects on the tissue can be evaluated using different techniques. Most authors performed similar assays to analyse the development of infection in the osteomyelitis mouse models. These techniques were classified into three categories: (i) quantification of the infection development, (ii) image analysis and (iii) histomorphological analysis.

\section{Quantification of the infection development}

To evaluate how the infection develops over time, it is advisable to perform a quantification of the number of bacteria present at different time points. 80 of the studies (59\%) evaluated the infection development by euthanising the animals at different time points, collecting bones, implants, surrounding tissues and/or organs and performing microbial culture to quantify the numbers of CFU (Kaur et al., 2016;
Nishitani et al., 2015b; Niska et al., 2012a; Tomizawa et al., 2020a; Young et al., 2015).

The quantification of infection can also be performed by qRT-PCR (Crane et al., 2009; Li et al., 2008; Wagner et al., 2016). This method allows distinguishing and quantifying between different bacterial species and is even considered to discriminate metabolically active and dormant bacteria.

Other authors used a non-invasive in vivo quantification method with BLI of bacteria to follow the infection development in single animals over time. The use of bioluminescent bacteria means that fewer animals are needed because the same animal can, in principle, be used to assess the degree of the infection at every time point over the course of the experiment (Bernthal et al., 2010; Li et al., 2008; Pribaz et al., 2012). One limitation of the bioluminescence technique is the loss of signal during the experiment due to the possible loss of the plasmid that carries the bioluminescence $l u x$ in the bacterial strain. Therefore, the use of a bacterial strain with lux inserted in the chromosome is advisable to not lose the bioluminescence signal over time. However, the bioluminescent signal can also disappear due to metabolic inactivity of the bacteria, which may be the consequence of biofilm formation. This will also happen with strains carrying $l u x$ in their chromosome. Therefore, this limitation should always be kept in mind when using bioluminescence monitoring of infections. The number of CFU, as derived from the bioluminescence readings at the last time point, should always be confirmed by comparison with the actual numbers of cultured CFU obtained from the specimens following animal sacrifice.

The following examples illustrate the possible difficulty in interpreting bioluminescence signals. The S. aureus ALC 2906 strain, which carries the bioluminescence construct on a chloramphenicolresistance-gene-containing plasmid, had a detectable bioluminescence signal at $10 \mathrm{~d}$ in a PJI mouse model. Then, the plasmid was lost due to the lack of pressure for antibiotic selection by chloramphenicol (Pribaz et al., 2012). On the other hand, S. aureus Xen 36 strain (ATCC 45925 as genetic background) had higher bioluminescence signals than Xen 40 strain (UAMS-1 as genetic background) but there were no differences in biofilm formation between the strains nor in numbers of CFUs cultured from the implant at $42 \mathrm{~d}$ post-infection (Pribaz et al., 2012). It is also important to consider that the bioluminescence signal intensity has been reported to be up to 10 -fold higher in BALB/C mice than in C57BL/6 mice due to the differences in light absorption in the respective white versus black pigmented skin and hairs of these mouse strains (Nishitani et al., 2015b).

A novel imaging modality based on ultrasound, known as photoacoustic imaging, was also used to follow the development of an infection (Wang et al., 2017a). In this technique, laser pulses are 
directed into the body at a specific wavelength to excite a photoacoustic tracer molecule present in the target tissue. The authors conjugated the tracer molecule indocyanine green to beta-cyclodextrin, a polysaccharide taken up by bacteria but not by host cells. Photoacoustic imaging provided significantly deeper tissue penetration (30-50 $\mathrm{mm}$ ) than BLI (10$20 \mathrm{~mm}$ ) (Wang et al., 2017a).

\section{Image analysis}

Advanced imaging techniques such as conventional radiography (Carli et al., 2018; Rochford et al., 2016), MCT (Niska et al., 2012a) and MRI (Horst et al., 2012) provide three-dimensional images of the bone structure. In most cases, these techniques were used to evaluate the structural effects of the infection in the bone (e.g. bone destruction and inflammation) and on the fracture healing process (Fig. 4d). These methods of visualisation were also used to check proper placement of the orthopaedic device upon implantation (Trombetta et al., 2019a). In addition, $\mu \mathrm{CT}$ scans can reveal detailed information about tissue mineral density, total callus volume and bone volume fraction of the callus, allowing for evaluation of the bone regeneration process after trauma or fracture. Although $\mu \mathrm{CT}$ scans can also be applied in vivo, ex vivo $\mu \mathrm{CT}$ scans provide a significantly higher resolution because ex vivo a higher dose of X-ray irradiation can be used (Bernthal et al., 2014; Laperre et al., 2011; Niska et al., 2012a).

MRI is one of the imaging modalities of choice for osteomyelitis diagnosis in humans because it provides excellent anatomical detail and it is noninvasive and highly sensitive for detecting an early infection. Horst et al. (2012) used MRI to monitor the development of the osteomyelitis during the acute phase of the infection in mice and its progression into a chronic infection. MRI allowed studying the presence of inflammation as well as the bone thickness and deformation in the mouse tibia (Horst et al., 2012).

Other imaging techniques used on ex vivo samples are SEM (16 \%), TEM (3\%) and confocal microscopy (1\%). Several authors have used SEM to evaluate the bacterial biofilm formation on the surface of the bone tissue or/and inserted devices (Carli et al., 2018; Sabaté Brescó et al., 2017; Trombetta et al., 2019a; Windolf et al., 2013). TEM can also be used to obtain bone tissue images with a higher magnification and resolution than SEM. For example, de Mesy Bentley et al. (2017) used TEM to assess and analyse the invasion of $S$. aureus into the bone and the remodelling of the bone tissue. Moreover, the use of TEM has shown the formation of $S$. aureus colonies within the non-mineralised collagen matrix and located intracellularly within neutrophils (Masters et al., 2021).

\section{Histomorphological analysis}

Histological staining methods were developed to assess and analyse the progression of infection in the bone and the remodelling process of the bone fracture and surrounding tissues (Rochford et al., 2016; Sabaté Brescó et al., 2017; Windolf et al., 2013; Yokogawa et al., 2018). This technique can identify bacteria and distinguish between different cell types such as osteoblasts, osteoclasts and neutrophils as well as between structural features of the infected tissue. These structural changes in the tissues are well-established for humans and are used to diagnose osteomyelitis in clinical practice.

The histological examination of the osteomyelitis mouse model developed by Horst et al. (2012) was performed during the acute and chronic phase of the osteomyelitis and compared to histological tissue samples from a patient with acute osteomyelitis and a patient with chronic osteomyelitis. The histological sections revealed a massive influx of granulocytes, with an intense bacterial colonisation and necrosis, during acute infection at $7 \mathrm{~d}$ that was similar to that seen in the human acute osteomyelitis sections. At $21 \mathrm{~d}$, in the stage of chronic infection, the mouse sections revealed osteoclastic resorption with new bone formation similar to the human chronic osteomyelitis sections (Horst et al., 2012). Another osteomyelitis mouse study, that used histological staining, included a scoring system of the sections according to the level of cells infiltration, with scores of 0 for no osteomyelitis, 1 for minimal or rare $(<10 \%$ tissue involvement), 2 for mild (10-20\%) and 3 for frequent (20-50\%) osteomyelitis (Lee et al., 2002). Haematoxylin and eosin straining was performed to stain tissue and cells, Gram staining for bacteria. Similarly, Büren et al. (2019) developed a standardised histological score system for a bone-fracture-related infection model to evaluate the severity of the infection in the mouse. Their score system evaluated 4 independent histological parameters: (i) presence of callus, (ii) consolidation of the osteotomy, (iii) structural changes of the medullary cavity, (iv) number of bacteria. The presence of callus and the consolidation of the osteotomy were evaluated using haematoxylin-eosin staining and the quantification of bacteria was performed following Giemsa staining (Büren et al., 2019). These examples illustrate the usefulness of histological scoring systems for a semi-quantitative evaluation of histology in mouse osteomyelitis models.

\section{Literature review of osteomyelitis mouse models}

135 osteomyelitis infection mouse studies were collected, analysed and classified into the following 5 categories: (i) haematogenous osteomyelitis (11\%), (ii) post-traumatic osteomyelitis (22\%), (iii) bone-implant-related infection (28\%), (iv) PJI (26\%), (v) fracture-related infection (13\%) (Fig. $5 a)$. The haematogenous osteomyelitis models are characterised by the bacterial or fungal colonisation of the bone through the bloodstream. The posttraumatic osteomyelitis models represent the local infection of the bone following unicortical defect or trauma. The bone-implant-related infection models 
mimic the development of an infection in the presence of a foreign body in the bone. The PJI models mimic the development of an infection in the presence of a device in the bone and synovial fluid. The fracturerelated infection models represent the development of an infection in a bone fracture. The authors studied the course of the infection either in the femur (58\%), tibia (37\%), femur and tibia (2\%), humerus $(1 \%)$ or radius $(1 \%)$ (Fig. 5 b). The mouse femur was selected more often than the tibia, for several reasons. The femur is tubular, larger and thicker than the tibia, thus facilitating the insertion of large devices. Moreover, the curvature of the tibia may complicate the insertion of longer devices. Lastly, the proximity of the fibula to the tibia can influence the development of the infection and the healing of the bone.

\section{Haematogenous osteomyelitis mouse models}

Haematogenous osteomyelitis takes place following a symptomatic or asymptomatic bloodstream infection that allows the pathogen to reach the bone. Worldwide incidence of haematogenous osteomyelitis is 1:1,000-20,000 people, with half of the cases occurring in children younger than 5 years (Popescu et al., 2020).

Generally, microorganisms will arrive at the metaphyses of long bones (i.e. femur or tibia), since these are highly vascularised. The lower fluid flow at the bone metaphysis allows the microorganism to establish an infection and cause local inflammation. In the haematogenous mouse infection models' studies reviewed, the inoculum volume used to establish the infection was between 100 and $150 \mu \mathrm{L}$ and a dose between $10^{6}$ and $10^{8} \mathrm{CFU}$, with the inoculum administered by injection via the retro-orbital venous sinus, intravenously or via the lateral tail vein (Table 1; Fig. 6a). $33 \%$ of the bone haematogenous infection studies evaluated the presence of infection in the femur, $40 \%$ in the tibia and $20 \%$ in both tibia and femur (Fig. 7a). In most of the haematogenous studies, no trauma or fracture was caused nor was a device inserted into the bones of the animals before the inoculation. There were 2 studies where a fracture or a trauma was caused before the inoculation of the infection (Chadha et al., 1999; Yoon et al., 1999) and in

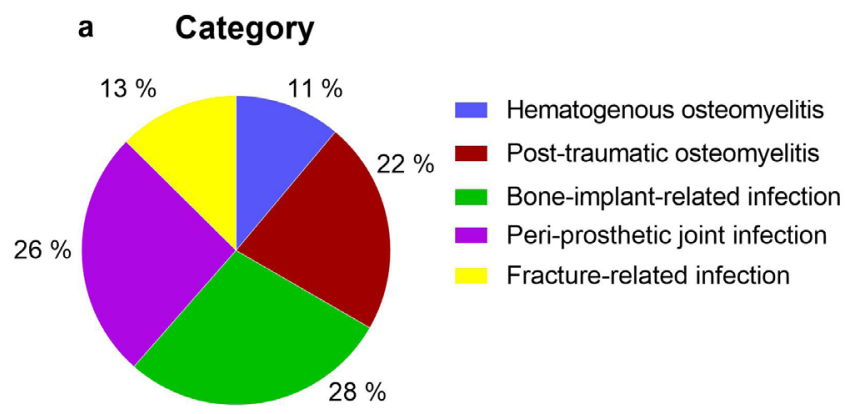

Total $=135$
1 study a haematogenous infection in a bone implant was studied (Wang et al., 2017c).

A haematogenous osteomyelitis model was developed to study the contribution of $S$. aureus Cna to the capacity of the bacteria to reach the bone from the bloodstream and cause an osteomyelitis (Elasri et al., 2002). The authors infected NIH Swiss mice with a $100 \mu \mathrm{L}$ inoculum containing $10^{8} \mathrm{CFU}$ of $S$. aureus UAMS-1 or its isogenic Cna mutant strain UAMS-237 injected via the lateral tail vein. Their results showed that the mutation of Cna limited the capacity of $S$. aureus to cause osteomyelitis through the haematogenous route. Another study with a similar set up $\left(150 \mu \mathrm{L}\right.$ inoculum of $10^{6} \mathrm{CFU}$ of $S$. aureus ATCC 53657 injected in the lateral tail vein) described a biphasic development of the bacterial infection. There was an acute phase of infection in the tibiae during the first 2 weeks followed by a chronic phase until the termination at $60 \mathrm{~d}$ (Horst et al., 2012).

Tuchscherr et al. (2017) studied the role of sigB in the development of a chronic osteomyelitis infection. They used a mouse model based on studies by Horst et al. (2012) and infected the animals with $150 \mu \mathrm{L}$ of $10^{6}$ CFUs via the lateral tail vein. Mice were infected with $S$. aureus LS1, S. aureus LS1 with $\operatorname{sig} B$ deleted and $S$. aureus LS1 sigB-deleted strain complemented for this deletion. Their results demonstrated the importance of $\operatorname{sig} B$ to establish osteomyelitis through the haematogenous route (Tuchscherr et al., 2017).

Wang et al. (2017c) developed a well-described model to mimic a haematogenous infection with an implanted device. They used male C57BL/6 mice and inoculated $S$. aureus SAP231 intravenously through the retro-orbital sinus $21 \mathrm{~d}$ after the implantation of a pin in the bone. All mice challenged with either $10^{6} \mathrm{CFU}$ or $5 \times 10^{6} \mathrm{CFU}$ inoculum survived the bacterial challenge. An inoculum of $10^{7} \mathrm{CFU}$ was too large and killed several animals. However, not all the inoculated mice developed the hematogenous implant infection because at $28 \mathrm{~d}$ post-inoculation there were no detectable CFU from the implants or surrounding tissues (Wang et al., 2017c).

In general, bone haematogenous infection models were successfully developed in the mouse and allowed for studying the essential bacterial features to establish an acute or chronic bone infection.

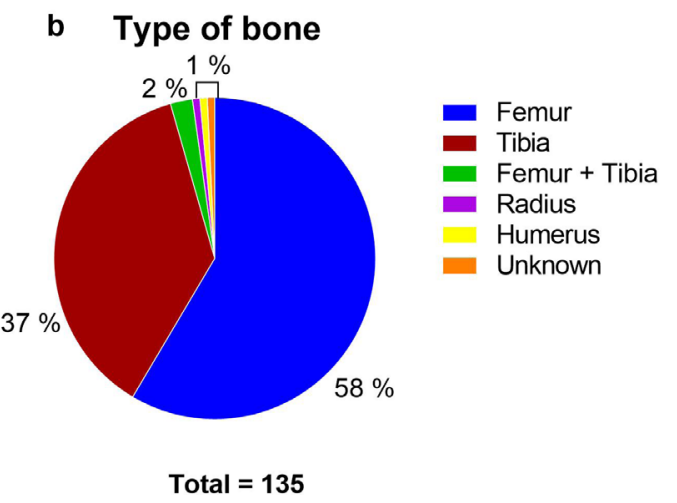

Fig. 5. Classification of the osteomyelitis mouse models by (a) categories and by (b) type of bone infected. 135 studies were included in the graphs. 
Post-traumatic osteomyelitis mouse models

Osteomyelitis can occur after trauma even without the insertion of a fixation device (Table 2; Fig. 6b). The incidence of osteomyelitis following open fractures is reported to be $1-30 \%$, depending significantly on the grade of trauma and the type of treatment administered (Metsemakers et al., 2015).

The post-traumatic osteomyelitis studies that have been included in this review have in common the creation of a unicortical bone defect and the local infection of the defect with 0.5 to $2 \mu \mathrm{L}$ of bacterial inoculum containing $10^{5}$ to $10^{8} \mathrm{CFU}$ as inoculum. Such a small volume of inoculum is recommended to prevent the diffusion of the inoculum to the contiguous tissues and to better mimic the real situation occurring in patients. The unicortical defect was a perforation only affecting one lateral side of the bone, with a size that ranged from 0.5 to $1.5 \mathrm{~mm}$ of diameter (Table 2, Fig. 6b). In $57 \%$ of the posttraumatic osteomyelitis studies the defect was created in the mouse femur and in $43 \%$ in the tibia (Fig. 7 b).

The post-traumatic osteomyelitis mouse models were used to study different virulence factors involved in the pathogenesis of the osteomyelitis as described previously (Cassat et al., 2013; Isogai et al., 2020; Loughran et al., 2016; Wilde et al., 2015; Xiao et al., 2017). The models were also used to study the effects of the immune response in clearing

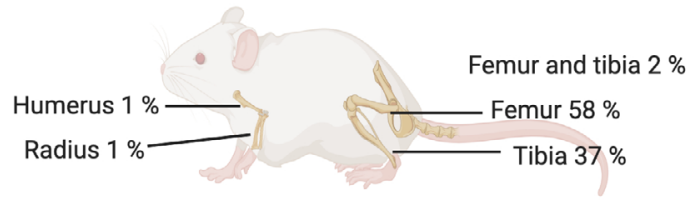

a Haematogenous osteomyelitis

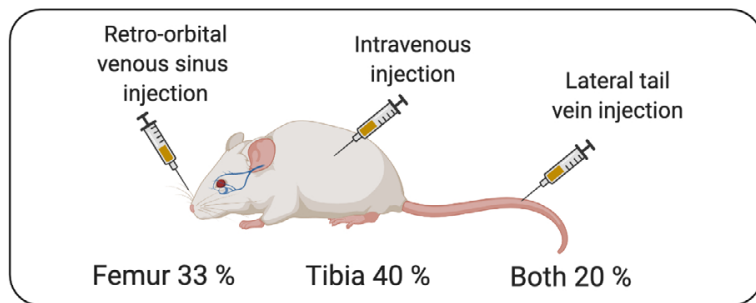

b Post-traumatic osteomyelitis

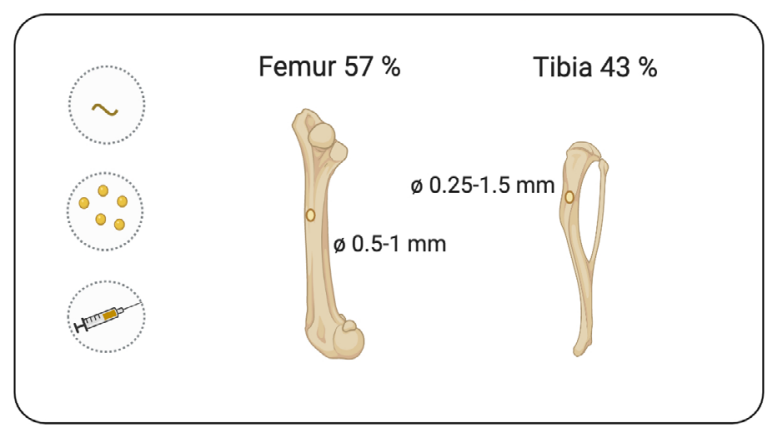

c Bone-implant-related infection

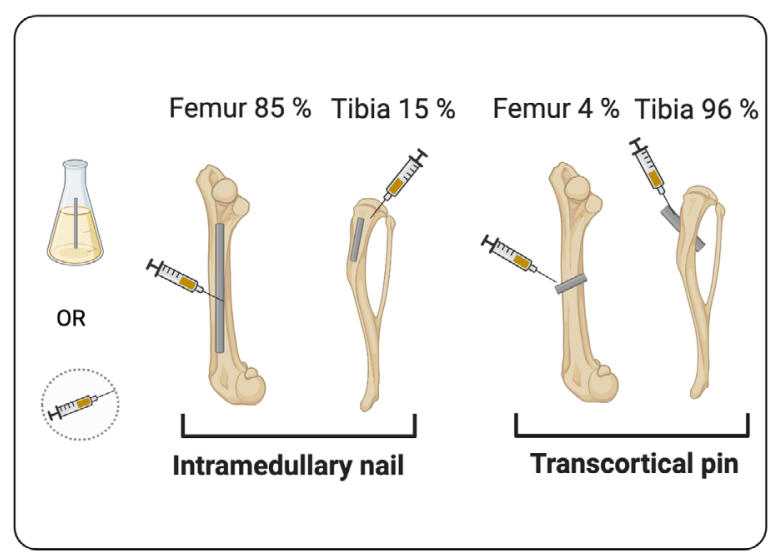

Inoculation methods legend

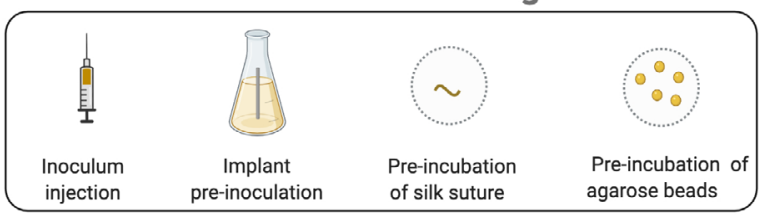

d Peri-prosthetic joint infection
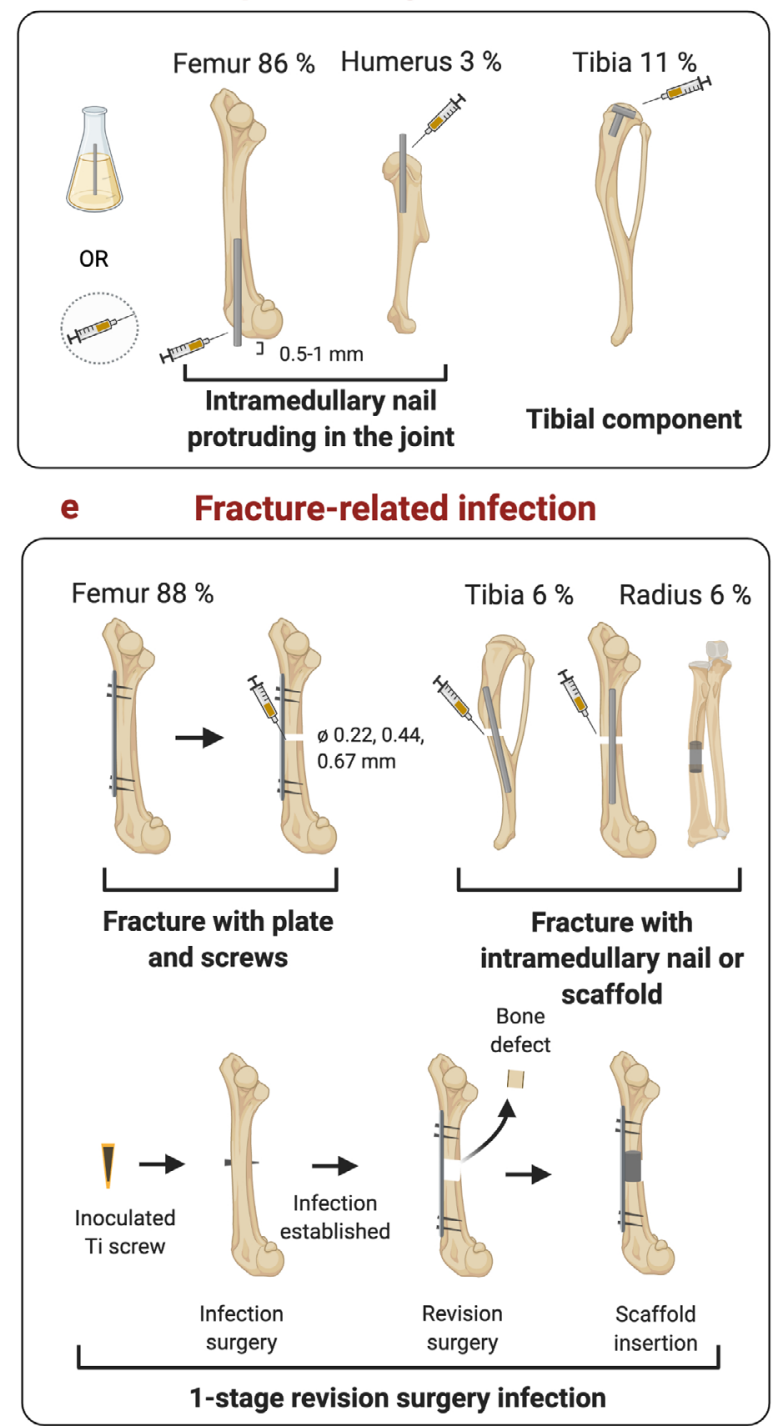

Fig. 6. Schematic representation of mouse models to induce bone infections and osteomyelitis based on the results of the literature search. (a) Haematogenous osteomyelitis, (b) post-traumatic osteomyelitis, (c) bone-implant-related infection, (d) PJI, (e) fracture-related infection. Ø: diameter. 
the infection and in the repair of the bone damage (Chen et al., 2017; Marriott et al., 2004; Putnam et al., 2019; Wagner et al., 2019; Yoshii et al., 2002b). For example, Marriot et al. (2004; 2005) developed a trauma-induced staphylococcal osteomyelitis model to study the inflammatory response of osteoblasts to infection. They created a bone defect in the bone cortex, inoculated with $10^{3} \mathrm{CFU}$ of $S$. aureus UAMS1 in agarose beads, and evaluated inflammatory mediators' levels for $4 \mathrm{~d}$. An increase in the level of the cytokines IL-6 and MCP1 by the osteoblasts was measured in the animals infected with $S$. aureus. The positive recruitment of macrophages by the MCP1 to clear the infection also had the negative consequence of an increased inflammation with bone damage (Marriott et al., 2004; 2005). An increase in the levels of IL-6 was also observed in a study where a silk suture, pre-inoculated with $10^{5} \mathrm{CFU}$ of a clinical isolate of $S$. aureus, was inserted into the tibia of ICR mice (Yoshii et al., 2002b). This resulted in a local elevated level of the cytokines IL- 6 and IL- $1 \beta$ in the early phase of the infection related to the bone damage, which was followed by an increase in TNF $\alpha$ and IL-4 in a later phase of the infection (Yoshii et al., 2002b). Putnam et al. (2019) studied the host immune pathways that contribute to bacterial immunity but also to bone destruction during $S$. aureus osteomyelitis. They created a bone defect in C5BL/6J wild type and a Haematogenous osteomyelitis

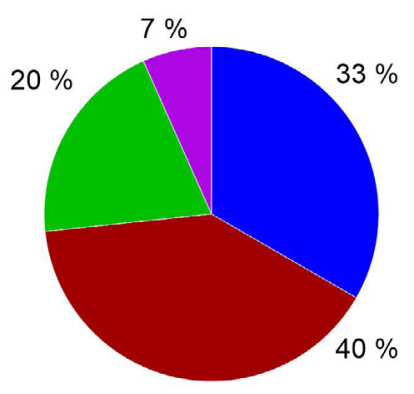

Total $=15$

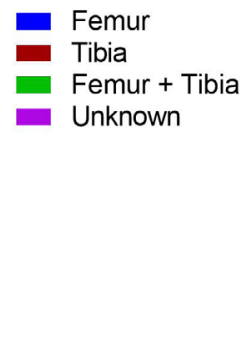

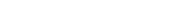

\section{b Post-traumatic osteomyelitis}

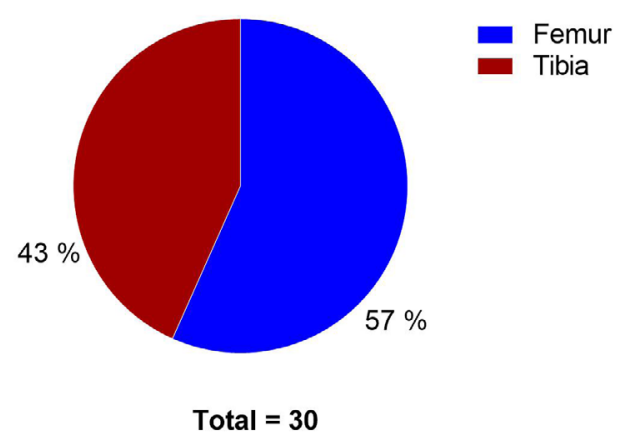

d Prosthetic joint infection

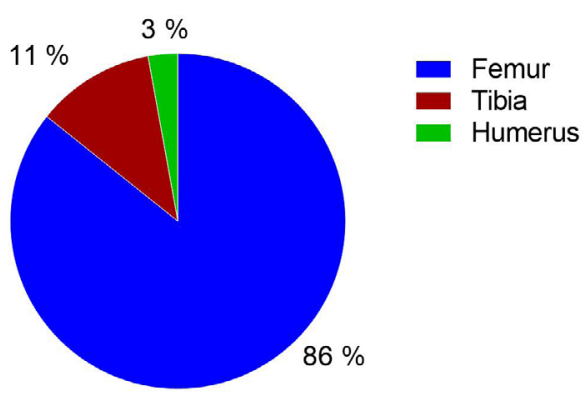

Total $=35$

e Fracture-related infection

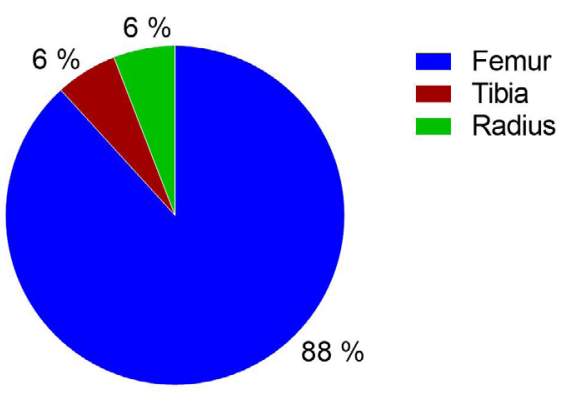

Total $=17$

Fig. 7. Type of bone infected (tibia, femur or both) in each category. (a) Haematogenous osteomyelitis, (b) post-traumatic osteomyelitis, (c) bone-implant-related infection, (d) PJI, (e) fracture-related infection. 135 studies were included in the graphs. 
in $\mathrm{MyD}^{-/-}$and IL1r1 ${ }^{-/-}$knock-out mice, infected them with a $2 \mu \mathrm{L}$ inoculum containing $10^{6} \mathrm{CFU}$ of S. aureus AH1263 strain and measured cytokine levels after $14 \mathrm{~d}$. They discovered an essential role of IL-1R to control the local bacterial replication during osteomyelitis and IL-1R contribution to bone destruction. Specifically, they demonstrated that there is an increase in the numbers of osteoclasts residing on the bone surface during the infection (Putnam et al., 2019).

The post-traumatic osteomyelitis models were used to evaluate the efficacy of antimicrobial and anti-inflammatory strategies to prevent and treat both bone infection and inflammation (Funao et al., 2012; Lu et al., 2019; Takahashi et al., 2008; Wagner et al., 2016; Wu et al., 2018; Zhu et al., 2019). Funao et al. (2012) established a real-time, quantitative and reproducible osteomyelitis mouse model using the bioluminescent bacterial strain $S$. aureus Xen 29. They perforated the femur to simulate an osteotomy, injected a $1 \mu \mathrm{L}$ inoculum containing $10^{8} \mathrm{CFU}$ in the medullar cavity and followed the infection in vivo (Funao et al., 2012). Wagner et al. (2016) created a mouse model to analyse bone loss and regeneration following $S$. aureus bone infection and treatment. They inoculated C57BL/6 mice tibiae with $2 \times 10^{3} \mathrm{CFU}$ of $S$. aureus Rosenbach 1884 and, after $14 \mathrm{~d}$, performed debridement and applied antibiotic therapy. The treatment group showed complete eradication of the bacteria, whereas the infection persisted in the control group (Wagner et al., 2016). Wu et al. (2018) used a post-traumatic osteomyelitis mouse model to evaluate the efficacy of the immune modulator baicalin to prevent bone destruction by regulating the toll-like receptor 2 . They inoculated BALB/c male mice with $1 \mu \mathrm{L}$ of $10^{8} \mathrm{CFU}$ of $S$. aureus ATCC 43300 with or without baicalin and showed that baicalin reduced the destruction of the bone (Wu et al., 2018). Lu et al. (2019) developed a bone defect infection mouse model in ICR/JCL with $2 \mu \mathrm{L}$ of $10^{6} \mathrm{CFU}$ of $S$. aureus ATCC 6538 to evaluate the efficacy of intravenous injection of the antibiotic gentamicin combined with a photodynamic therapy as a treatment for osteomyelitis (Lu et al., 2019).

In conclusion, post-traumatic osteomyelitis mouse models can be used to elucidate the pathophysiology of the bone infection. They can be used to study the influence of the bacterial virulence factors or the influence of the infection in the immune system activation causing an increased bone damage.

\section{Bone-implant-related infection mouse models}

The presence of a foreign body, such as a bone implant, increases the risk of infection because the implant acts as a niche for bacterial colonisation and compromises the local immune response against the bacteria. An established bone-implant-related infection mouse model to study the pathophysiology of the bone implant infection is the intramedullary implant model (Table 3; Fig. 6c). This method basically consists of the implantation of a K-wire, a pin or a nail into the intramedullary canal of the femur or tibia, combined with a bacterial inoculum (Table 3, Fig. 6c). Other studies have described the transcortical insertion of a pin into the femur or tibia that in some cases was bent at both ends to increase the device stability (Jørgensen et al., 2014; Li et al., 2008; Prabhakara et al., 2011a). The bacterial inoculum was applied directly into the femoral or tibial insertion site to mimic a contamination during surgery (Funao et al., 2016). Alternatively, the device was pre-inoculated before insertion into the bone to mimic the contamination of the device (Jørgensen et al., 2014). In $68 \%$ of the studies, the implant was inserted in the tibia of the animals and in $32 \%$ in the femur (Fig. 7c). These bone-implant-related infection mouse models were used to study different pathophysiological mechanisms of osteomyelitis related to bone implants. For example, a recent study revealed that $S$. aureus bacteria can colonise very small spaces in the bone such as canaliculi and osteocyte lacunae of cortical bone, sites in the bone which are difficult for immune cells to reach (de Mesy Bentley et al., 2017). These models were also applied to study different pathways and aspects of the immune response to the infection and the implant (Prabhakara et al., 2011a; Prabhakara et al., 2011b).

The models were also extensively used to evaluate the efficacy of antimicrobial compounds administered either systemically (Jørgensen et al., 2016; Li et al., 2008) or locally at the site of infection (Crane et al., 2009; Funao et al., 2016; Wells et al., 2018; Zhang et al., 2019a). Novel strategies for preventing or treating infection, such as passive immunisation (Varrone et al., 2014), hyperbaric oxygen therapy (Jørgensen et al., 2017; Shandley et al., 2012) and antimicrobial peptides (Choe et al., 2015), were also evaluated using these models.

In conclusion, the bone implant infection models allow for the study of the different interactions that occur between implant, infection and immune response in the bone environment and can be used to evaluate the efficacy of antimicrobials administrated systemically or locally at the implant site. However, in these models there is no fracture nor device replacement to enable the study of the healing process in the presence of infection and implant. The fracture itself also influences the clearance of the infection. Hence, the model does not allow for studies on revision surgery with debridement and lavage or for evaluating antimicrobial devices used to treat infected bone tissue after removal of a contaminated device.

Bone-implant-related infections in diabetic mouse models Diabetes is one of the most relevant risk factors in implant-related infection and its prevalence is growing worldwide (Berendt et al., 2008). Diabetes is a disease in which high blood glucose levels occur. It can be due to a loss of the insulin-producing $\beta$-cells in the pancreas islets, known as T1D, or to insulin resistance due to a glucose-enriched diet, known as T2D (Farnsworth et al., 2015). Both T1D 
and T2D animal models are used to study diabetic complications, such as osteomyelitis, and their potential treatment.

Lovati et al. (2013) were the first to describe a diabetic bone-implant-related infection mouse model (Table 4). They compared the development of bone implant infection in the T1D diabetic mouse strain diabetic NOD/ShiLtJ and in non-diabetic CD1 mice (Lovati et al., 2013). Their results showed that the same bacterial inoculum of $10^{3} \mathrm{CFU}$ of $S$. aureus ATCC 25923 induced bone-implant infection in the diabetic mice but not in the wild type mice. This animal model may be a useful tool for in vivo testing of treatment strategies in diabetic and non-diabetic individuals. Moreover, the authors showed the synergistic effects of the vasodilator PGE1 with an antibiotic, as a cotreatment for bone-implant infection in diabetic mice (Lovati et al., 2014).

Farnsworth et al. (2015) studied the risk factors of orthopaedic infections for diabetic patients by comparing the development of bone implant infection and adaptative immunity in T1D and T2D male C57BL/6J mice. The T1D model was induced using a toxic agent that selectively destroys the beta pancreatic cells (i.e. streptozotozin) eliminating the insulin production. The T2D model consisted of 5 week old mice that received a high-fat diet $(60 \%$ $\mathrm{kcal})$. To confirm the hyperglycaemia, the glucose levels were measured at several time points. Then, the investigators inserted a surgical wire into the tibia of the animals that had already been inoculated with S. aureus Xen 36. Results showed that T2D mice had a more severe $S$. aureus osteomyelitis than T1D mice (Farnsworth et al., 2015).

\section{PJI mouse models}

PJI following total joint arthroplasty of the hip or knee, despite the low incidence of $1-2 \%$, is still associated with a large burden in orthopaedic surgery. The PJI mouse models' studies considered in the present review principally describe the insertion of a pin into the intramedullary canal that protrudes 0.5-1 mm into the joint (Table 5, Fig. 6d, Heim et al., 2014; Heim et al., 2015a; Kaur et al., 2016; Thompson et al., 2017; Thompson et al., 2018). To better mimic the PJI, other studies have developed the tibial component of the knee prosthesis and press-fitted this into the tibia, exposed to the joint space (Carli et al., 2017; Carli et al., 2018; Hernandez et al., 2019). In all the studies, the bacterial inoculum was pipetted or injected into the joint tissue or space to reproduce the principal clinical aetiology through intraoperative contamination (Table 5). $86 \%$ of the studies inserted the device into the femur of the animals, $11 \%$ into the tibia and $3 \%$ into the humerus (Fig. 6d, 7d).

Bernthal et al. (2010) model provided real-time longitudinal tracking of the infection in a postsurgical joint for $10 \mathrm{~d}$ by using a bioluminescent $S$. aureus strain and monitoring the inflammation with the use of a mouse line that possesses fluorescent neutrophils, the LysEGFP knock-in mice. They showed a more acute joint infection when the mice were inoculated with $5 \times 10^{3}$ or $5 \times 10^{4} \mathrm{CFU}$ versus a mild infection, resembling a chronic infection, following challenge with a bacterial inoculum of $5 \times 10^{2}$ CFU. Mice inoculated with $2 \mu \mathrm{L}$ containing either $5 \times 10^{3}$ or $5 \times 10^{4} \mathrm{CFU}$ developed an increased bacterial bioluminescent signal corresponding to increased bacterial numbers and marked swelling of the affected leg within $3 \mathrm{~d}$, consistent with an acute joint infection. In contrast, mice inoculated with $5 \times 10^{2} \mathrm{CFU}$ developed a low-grade infection, resembling more a chronic infection (Bernthal et al., 2010). This model was further used to compare infection development and biofilm formation with different S. aureus strains (Pribaz et al., 2012) or by extending the experiment to even $40-49 \mathrm{~d}$ to study a chronic infection (Niska et al., 2012a; Pribaz et al., 2012). Interestingly, the studies described a peak in bioluminescence for bacteria as well as neutrophils at $3 \mathrm{~d}$, corresponding to the acute phase of the infection, and bioluminescence values decreasing over time, corresponding to the chronic phase of the infection. As previously mentioned, a decrease in luminescence does not necessarily correspond to a reduction in bacterial numbers but may well be associated with a lower metabolic activity of the bacteria in the later, chronic stages of infection (Pribaz et al., 2012). To provide deeper tissue penetration, a photoacoustic imaging system was applied to monitor the infection in this type of mouse model (Wang et al., 2017a). However, this method was only used to study a $4 \mathrm{~d}$ infection.

Sheppard et al. (2020) developed a mouse model with an implant in the humerus that was also protruding into the joint. The mouse humerus and femur have a similar diameter. In this model, two inoculum doses where evaluated: a lower inoculum of $10^{3} \mathrm{CFU}$ that caused mild osteolysis and a higher inoculum of $10^{4} \mathrm{CFU}$ that caused severe osteolysis and failure of the shoulder.

These models were also extensively used to evaluate the efficacy of antimicrobial compounds administered either systemically (Niska et al., 2013) or locally at the site of infection (Bernthal et al., 2010; Carli et al., 2017; Stavrakis et al., 2016; Stavrakis et al., 2019; Thompson et al., 2017). In a comparative study, local antibiotic administration was shown to be more efficacious than systemic administration (Young et al., 2015).

In summary, the PJI mouse models described in this section can reproduce the environment of the clinical condition by either incorporating the prosthetic device into the joint or protruding into joint and inoculating the infection into the joint. However, the small size of the mouse hampers the development of all the parts of the prosthetic device that are used for PJI human patients.

\section{Fracture-related infection mouse models}

A bone fracture is usually fixed using an intramedullary nail or a fixation plate with bone 
screws. The presence of an infection will compromise the immune response and the regeneration of the bone. The incidence of an infection after the fixation of a fracture ranges from $1 \%$ in closed fractures to $30 \%$ in complex open tibial fractures (Metsemakers et al., 2015).

To mimic this clinical situation, fracture-related infection mouse models fix a fracture using a fixation plate or an intramedullary nail. In the studies that fix the fracture using a fixation plate and screws, the fixation plate is applied to the long bone with screws, followed by fracture or removal of a part of the bone and challenge with a bacterial inoculum (Table 6, Fig. 6e). All the studies using fixation plates apply surgical devices such as the "MouseFix" plate with 4 or 6 screw holes and/or surgical tools such as a Gigli saw to create a fracture size diameter of 0.22 , 0.44 or $0.67 \mathrm{~mm}$ (RISystem, Davos, Switzerland). Alternatively, the studies that fix the fracture using an intramedullary nail, create the fracture first and then ream the bone pieces using a nail (Cahill et al., 2021; Johnson et al., 2018). $88 \%$ of the studies analysed created the bone fracture in the femur, $6 \%$ in the tibia and $6 \%$ in the radius (Fig. 6e, 7e).

Rochford et al. (2016) used this type of model to study the immune response during healing in the presence of an infection. They pre-inoculated the fixation device, 20 min before surgery, with $10^{5} \mathrm{CFU} /$ implant of S. aureus JAR 06.01.31. Sabaté-Bresco et al. (2017) used the same model and showed that stable fracture fixation led to better clearance of infection than unstable fixation. Moreover, the osteotomy gap had healed in the animals with the stable fixation by 30 d post-surgery (Sabaté Brescó et al., 2017).

In clinical practice, depending on the infection severity, there are different surgical approaches to treat an infection, such as one-stage or two-stage revision. A one-stage revision consists of one surgical intervention, with implant retraction, debridement and implantation of a new prosthesis. A two-stage revision consists of two surgical interventions: implant retraction, debridement and wound closure in the first stage surgery and, after several weeks, implantation of a new, cemented prosthesis during the second stage surgery. Moreover, a two-stage revision can also consist of implant removal with debridement and application of local antibiotics (spacers/beads) in the first stage and implant placement with antibiotic-loaded bone cement in the second stage.

Different parts of these revision-surgery approaches were mimicked in osteomyelitis mouse studies. For instance, the stage of revision surgery due to infection in the bone fracture fixation infection mouse model was first introduced by Windolf et al. (2013; 2014). The authors created a bone fracture fixation mouse model, with lavage and debridement of the infection at 7 and $14 \mathrm{~d}$ post-implantation and infection (Windolf et al., 2013). In a later study, they used this model to evaluate the treatment efficacy of a PMMA plate coated with the metallo-endopeptidase lysostaphin to prevent $S$. aureus infection (Windolf et al., 2014). The model was also used by others to evaluate the efficacy of other antimicrobial materials such as a calcium sulphate withgentamicin bone filler (Oezel et al., 2019) or hyperbaric oxygen therapy (Büren et al., 2019).

Other authors have developed models with hardware exchange to mimic one-stage revision surgery. For example, Inzana et al. (2015b) established a mid-diaphyseal femoral osteotomy repaired using a fixation device that was pre-inoculated with a collagen sheet containing $S$. aureus to initiate the infection. At $7 \mathrm{~d}$ post-infection, revision surgery with debridement and placement of an antibiotic-laden spacer was performed and, at $28 \mathrm{~d}$ post-infection, the efficacy of this procedure was evaluated. Yokogawa et al. (2018) established a similar model to investigate the synergy between systemic vancomycin treatment with immunotherapy. They pre-inoculated a bone screw with $S$. aureus and inserted it into the middle of the femur shaft to establish an infection.

Trombetta et al. (2019a) compared one- and twostage revision surgery using a more complex in vivo model that more closely resembles the clinical situation. They pre-inoculated a bone screw with S. aureus Xen 36 and placed it in the middle of the femur for $7 \mathrm{~d}$ to establish an infection. Then, they removed the infected hardware and introduced a fixation device and a PMMA spacer with or without local antibiotic delivery. The delayed treatment of the infection, described in the studies above, is a relevant clinical feature that increases the challenge of efficient bacterial clearance.

Regarding the healing of the fracture, several authors have shown - using X-ray or $\mu \mathrm{CT}$ - a complete bone regeneration in the non-infected group when compared to an infected group of animals (Sabaté Brescó et al., 2017; Windolf et al., 2013; Windolf et al., 2014). Moreover, the bone healing was also observed after $28 \mathrm{~d}$ post-osteotomy in the treatment group infected (Windolf et al., 2014). Thus, the evaluation of bone healing indicates the progress in the eradication of the infection and osteomyelitis.

To summarise, fracture-related infection models allow the creation of procedures to study a wide spectrum of clinical situations and can be used to study new strategies for the prevention and treatment of osteomyelitis related to orthopaedic bone fixation devices.

\section{Advantages, limitations and conclusions}

The advantages of the osteomyelitis mouse models are the similarity of their bone physiology to that of humans, their relatively low cost, the availability of well-defined inbred strains and molecular and immunological tools as well as the possibility of genetically modifying them.

Mouse models have proven to be adaptable to specific desired model conditions, evolving 
from simpler models, such as the post-traumatic osteomyelitis (Funao et al., 2012), through the bone-implant infections (Li et al., 2008), until more complex models such as the fracture-related infection with hardware exchange (Trombetta et al., 2019a; Yokogawa et al., 2018). Thus, these in vivo mouse models can be applied to study a variety of research questions related to osteomyelitis infection.

Larger animal models such as rabbits, dogs or sheep show more similarities to humans than mouse in their bone microstructure, macrostructure and bone remodelling process (Muschler et al., 2010; Pearce et al., 2007; Wancket, 2015) and allow the use of implants of a size more closely resembling the actual implants used for humans. Moreover, larger animals such as dogs can tolerate multiple surgical procedures, allowing researchers to study some of the more complex procedures that are performed in humans (Patel et al., 2009). However, larger animals are more expensive, as is their maintenance, and they take longer to reach skeletal maturity than mice (Patel et al., 2009; Pearce et al., 2007).

The use of GM mouse strains and molecular tools allows performing detailed studies on pathways and cells involved in the different mechanisms and processes of a disease, such as the inflammatory response to infections and fractures as well as the healing process. For example, Bernthal et al. (2014) used a GM mouse strain with fluorescent neutrophils to follow in vivo the inflammatory response to the infection and the presence of the implant. GM mice were also very useful for creating an osteomyelitis diabetic mouse model (Farnsworth et al., 2015). Compared to larger animals, a wide range of mice GM lines is available, permitting the study of different aspects of osteomyelitis (Pearce et al., 2007).

Mouse models are also relevant to developing new preventive and treatment strategies to control infections. For example, antimicrobial devices might be a promising approach to reduce infections in orthopaedic surgery. Coatings of implants used for fracture stabilisation in rats or mice are less expensive than those used in large animals such as sheep or goats. In fact, many authors have well-described procedures to test the efficacy of antimicrobial coatings in osteomyelitis mouse models (Table 3-6). Such models can be used as a first step in the in vivo evaluation of in vitro results, to study the distribution of drugs in bones and other organs and to develop new antimicrobials for infection prevention. If the aim of using an antimicrobial device is to prevent an infection, it will be necessary to evaluate its in vivo antimicrobial efficacy for at least the first $10 \mathrm{~d}$. But, if the antimicrobial device is meant to be used as a treatment, a longer evaluation will be necessary (Moriarty et al., 2019). In any case, an antimicrobial orthopaedic device must prove its antimicrobial efficacy against relevant Gram-positive and Gram-negative pathogens (such as multidrugresistant bacteria), drug release, lack of inducing excessive inflammatory responses, prevention of bone tissue damage and support of bone healing. The antimicrobial efficacy should be determined by quantification of CFUs on the implant as well as in the bone tissue and surrounding soft tissue at relevant time points. Moreover, the inflammatory response to the device can be determined by measuring the cytokines in the tissue and performing immunohistology. Destruction of the bone tissue can be evaluated using imaging techniques such as $\mu \mathrm{CT}$ or MRI.

Most of the studies analysed established an infection using $S$. aureus clinical strains because $S$. aureus is one of the major osteomyelitis pathogens (Fig. 3a). However, it is also relevant to study more in depth the pathophysiology of other devastating bacterial and fungal species related to those that cause osteomyelitis - such as coagulasenegative staphylococcal and streptococcal species, $P$. aeruginosa, C. acnes, A. baumannii, C. albicans - or polymicrobial infections (Bemer et al., 2014; Kavanagh et al., 2018).

The mortality rate related to these osteomyelitis mouse models varied between studies principally due to the differences in the infective dose, the virulence of the bacterial strain and the type of surgical procedure. Part of the information related to the mortality rates was retrieved from the publications and another part by consulting the authors of studies where no details on mortality were published.

The type of inoculum administration and the infectious dose applied in the animals will directly affect the animal mortality rate. For example, the administration of a localised low inoculum is not expected to cause an invasive spread of the infection (Lloyd Miller, personal communication, 2021). However, if a high infective dose is locally inoculated into the joint or bone, it could cause an invasive haematogenous spread leading to sepsis and, consequently, to the death of the animals (Lloyd Miller, personal communication, 2021). Therefore, it is important to include dose-determining studies to find the proper inoculum to administer to the animals, depending on the final aim of the study.

The haematogenous models with associated sepsis had a high mortality rate of $20-50 \%$ due to the highly infective doses administered that lead to the development of sepsis, infection of other organs or extreme weight loss that necessitated euthanasia (Horst et al., 2012; Lorena Tuchscherr, personal communication, 2021). The risk of sepsis development was also affected by the virulence of the bacterial strain used and the susceptibility of the mouse strain infected (Lorena Tuchscherr, personal communication, 2021). In the haematogenous implant study, 3 different bacterial inocula were used $\left(10^{5}\right.$ to $\left.10^{7} \mathrm{CFU}\right)$, where only the largest bacterial inoculum caused a mortality rate of $10 \%$ (Wang et al., 2017c).

The post-traumatic infection model, the bone implant infection model and the PJI model in some studies had a mortality rate ranging from 1 to $10 \%$ due to anaesthesia complications during the surgical 
procedure (Ford et al., 2020, Hernandez et al., 2019; Elysia Masters, personal communication, 2021; Tammy Kielian, personal communication, 2021). Other studies did not report any loss of animals with these types of mouse models (Alexandra Stavrakis, personal communication, 2021). In some studies, there was a range of animals used per group "such as 5-8 animals per group" (Ashbaugh et al., 2016), which was related to the numbers of animals available to undergo the surgical procedure on different days and not to the mortality rate (Lloyd Miller, personal communication, 2021).

The studies that used fracture-related infection models encountered more complications. In the models with a fracture and an intramedullary nail the mortality was ranging between 5 and $10 \%$ and it was related to surgical complications (Cahill et al., 2021; Andres García, personal communication, 2021). In the models with a fracture fixed using a fixation plate, the mortality was related to intraoperative complications (ranging between 2 and $29 \%$ ) such as implant misplacement (Sabaté Brescó et al., 2017), anaesthesia fatality (Oezel et al., 2019; Sabaté Brescó et al., 2017; Ceylan Windolf, personal communication, 2021; Fintan Moriarty, personal communication, 2021) or cardiopulmonary instability (Oezel et al., 2019). The fatalities were also related to post-operative complications such as fixation device failure (Oezel et al., 2019; Sabaté Brescó et al., 2017; Ceylan Windolf, personal communication, 2021), fracture of the bone (Fintan Moriarty, personal communication, 2021), secondary infections (Sabaté Brescó et al., 2017) or device failure during revision surgery (Trombetta et al., 2019b). On the other hand, several other studies did not have any losses of animals (Büren et al., 2019; Inzana et al., 2015a; Inzana et al., 2015b).

Several authors concurred that once the surgical procedure and the infective dose were wellestablished and the personnel well trained, the mortality encountered was predominately related to the anaesthetics used during the procedure.

Other important features to study in more detail are the bacterial virulence factors related to the disease including the bacterial immune evasion mechanisms to avoid the clearance of the pathogens. The role of the immune response in osteomyelitis is very relevant for future studies considering the importance of the macrophages and neutrophils in the clearance of the infection and in the regeneration of the bone tissue. Understanding the cellular and molecular pathways involved in the recruitment, differentiation and activation of monocytes seems to be a central axis of research in the osteo-immunomodulation response. The mouse models can also be used to investigate how antimicrobial and anti-inflammatory drugs should be applied to the bone to prevent or treat the infection and orchestrate the inflammatory response to enhance the regeneration of the bone tissue.

Additional types of clinical osteomyelitis could also be mimicked using mouse models. For example, Röntgen et al. (2010) developed an external fracture fixation mouse model without infection. An infection could be established in this model to study its influence on bone healing with this type of fracture fixation device. Another clinical osteomyelitis model that could be established is the two-stages exchange of contaminated internal fixation hardware. However, the small size of the mouse makes it more challenging to perform multiple surgical procedures. Another interesting aspect for further study is the influence of the microbiome in the predisposition to osteomyelitis and how e.g. probiotics could help to reduce the effects of the disease (Hernandez et al., 2019). A bone infection could also be merged with other clinical models to try to reproduce models closer to the patient situation. For instance, Zhang et al. (2019b) developed a mouse fracture model using 20 to 28 months old mice presenting a $P$. aeruginosa infection in the endotracheal tube. This mimics a hospital-acquired infection due to the endotracheal tube, as occasionally present in patients with a fracture. The next step would be to inoculate the endotracheal tube to cause sepsis and study the ability of pathogens such as $S$. aureus to infect a bone fracture and how to prevent it using prophylaxis. However, it can be foreseen that establishing such a challenging model will be difficult.

The standardisation of the models is highly relevant for study results to be reproducible and comparable between laboratories and to achieve a better translation to the clinic (Coenye et al., 2018; Muschler et al., 2010). Ideally, standardised models would need to be developed but the wide variety of conditions used in the different models (as described in the present review) indicate that standardisation may be difficult. To allow for full understanding of differences between studies, it is vital to provide very clear and detailed descriptions of the models. For instance, the mouse strain used, animal age, gender, and weight should always be reported. The use of a well-defined bacterial strain, inoculum volume and dose as well as method of administration is also important. In this respect, guidelines defining the minimum study information that needs to be reported would be very useful tools to achieve these goals (Allkja et al., 2020; Moriarty et al., 2019).

In conclusion, the mouse is an excellent first step in vivo model to study the pathogenesis, inflammation and healing process of osteomyelitis with or without implanted medical devices and to evaluate novel prophylaxis and treatment strategies.

\section{Acknowledgements}

Fig. 2,6 were created using BioRender.com. This research is part of the PRINT-AID consortium, which has received funding from the European Union's Horizon 2020 research and innovation program under the Marie Skłodowska-Curie grant agreement number 722467. The authors would like to thank Dr Lloyd Miller, Dr Lorena Tuchscherr, Elysia Masters, 
Dr Tammy Kielian, Dr Alexandra Stavrakis, Dr Andres García, Dr Ceylan Windolf and Dr Fintan Moriarty for their personal input in the discussion about the mortality rate of the osteomyelitis mouse models.

\section{References}

Adams S, Pacharinsak C (2015) Mouse anesthesia and analgesia. Curr Protoc Mouse Biol 5: 51-63.

Aguilera-Correa JJ, Garcia-Casas A, Mediero A, Romera D, Mulero F, Cuevas-López I, JiménezMorales A, Esteban J (2020) A new antibiotic-loaded sol-gel can prevent bacterial prosthetic joint infection: from in vitro studies to an in vivo model. Front Microbiol 10: 2935. DOI: 10.3389/fmicb.2019.02935.

Allkja J, Bjarnsholt T, Coenye T, Cos P, Fallarero A, Harrison JJ, Lopes SP, Oliver A, Pereira MO, Ramage G, Shirtliff ME, Stoodley P, Webb JS, Zaat SAJ, Goeres DM, Azevedo NF (2020) Minimum information guideline for spectrophotometric and fluorometric methods to assess biofilm formation in microplates. Biofilm 2: 100010. DOI: 10.1016/j.bioflm.2019.100010.

Arciola CR, Campoccia D, Gamberini S, Baldassarri L, Montanaro L (2005a) Prevalence of Cna, fnbA and $f n b B$ adhesin genes among Staphylococcus aureus isolates from orthopedic infections associated to different types of implant. FEMS Microbiol Lett 246: 81-86.

Arciola CR, An YH, Campoccia D, Donati ME, Montanaro L (2005b) Etiology of implant orthopedic infections: a survey on 1027 clinical isolates. Int J Artif Organs 28: 1091-1100.

Ashbaugh AG, Jiang X, Zheng J, Tsai AS, Kim WS, Thompson JM, Miller RJ, Shahbazian JH, Wang Y, Dillen CA, Ordonez AA, Chang YS, Jain SK, Jones LC, Sterling RS, Mao H-Q, Miller LS (2016) Polymeric nanofiber coating with tunable combinatorial antibiotic delivery prevents biofilmassociated infection in vivo. Proc Natl Acad Sci U S A 113: E6919-E6928.

Ashman RB, Papadimitriou JM (1991) Chronic osteomyelitis as a consequence of systemic Candida albicans infection. Immunol Cell Biol 69: 427-429.

Beamer WG, Donahue LR, Rosen CJ, Baylink DJ (1996) Genetic variability in adult bone density among inbred strains of mice. Bone 18: 397-403.

Bemer P, Plouzeau C, Tande D, Leger J, Giraudeau B, Valentin AS, Jolivet-Gougeon A, Vincent P, Corvec S, Gibaud S, Juvin ME, Hery-Arnaud G, Lemarie C, Kempf M, Bret L, Quentin R, Coffre C, de Pinieux G, Bernard L, Burucoa C, the Centre de Référence des Infections Osteo-articulaires du Grand Ouest (CRIOGO) Study Team (2014) Evaluation of 16S rRNA gene PCR sensitivity and specificity for diagnosis of prosthetic joint infection: a prospective multicenter cross-sectional study. J Clin Microbiol 52: 3583-3589.

Berendt AR, Peters EJG, Bakker K, Embil JM, Eneroth M, Hinchliffe RJ, Jeffcoate WJ, Lipsky BA,
Senneville E, Teh J, Valk GD (2008) Diabetic foot osteomyelitis: a progress report on diagnosis and a systematic review of treatment. Diabetes Metab Res Rev 24: S145-S161.

Bernthal NM, Pribaz JR, Stavrakis AI, Billi F, Cho JS, Ramos RI, Francis KP, Iwakura Y, Miller LS (2011) Protective role of IL-1 $\beta$ against post-arthroplasty Staphylococcus aureus infection. J Orthop Res 29: 16211626.

Bernthal NM, Stavrakis AI, Billi F, Cho JS, Kremen TJ, Simon SI, Cheung AL, Finerman GA, Lieberman JR, Adams JS, Miller LS (2010) A mouse model of postarthroplasty Staphylococcus aureus joint infection to evaluate in vivo the efficacy of antimicrobial implant coatings. PLoS One 5: e12580. DOI: 10.1371/journal. pone.0012580.

Bernthal NM, Taylor BN, Meganck JA, Wang Y, Shahbazian JH, Niska JA, Francis KP, Miller LS (2014) Combined in vivo optical and $\mu \mathrm{CT}$ imaging to monitor infection, inflammation, and bone anatomy in an orthopaedic implant infection in mice. J Vis Exp: e51612. DOI:10.3791/51612.

Blevins JS, Elasri MO, Allmendinger SD, Beenken KE, Skinner RA, Thomas JR, Smeltzer MS (2003) Role of sarA in the pathogenesis of Staphylococcus aureus musculoskeletal infection. Infect Immun 71: 516-523.

Boelens JJ, Dankert J, Murk JL, Weening JJ, van der Poll T, Dingemans KP, Koole L, Laman JD, Zaat SAJ (2000) Biomaterial-associated persistence of Staphylococcus epidermidis in pericatheter macrophages. J Infect Dis 181: 1337-1349.

Boles LR, Awais R, Beenken KE, Smeltzer MS, Haggard WO, Jessica AJ (2018) Local delivery of amikacin and vancomycin from chitosan sponges prevent polymicrobial implant-associated biofilm. Mil Med 183: 459-465.

Brodt MD, Ellis CB, Silva MJ (1999) Growing C57Bl/6 mice increase whole bone mechanical properties by increasing geometric and material properties. J Bone Miner Res 14: 2159-2166.

Broekhuizen CAN, de Boer L, Schipper K, Jones CD, Quadir S, Feldman RG, Dankert J, Vandenbroucke-Grauls CMJE, Weening JJ, Zaat SAJ (2007) Peri-implant tissue is an important niche for Staphylococcus epidermidis in experimental biomaterial-associated infection in mice. Infect Immun 75: 1129-1136.

Buitrago S, Martin TE, Tetens-Woodring J, BelichaVillanueva A, Wilding GE (2008) Safety and efficacy of various combinations of injectable anesthetics in BALB/c Mice. J Am Assoc Lab Anim Sci 47: 11-17.

Büren C, Hambüchen M, Windolf J, Lögters T, Windolf CD (2019) Histological score for degrees of severity in an implant-associated infection model in mice. Arch Orthop Trauma Surg 139: 1235-1244.

Cahill SV, Kwon H, Back J, Lee I, Lee S, Alder KD, Hao Z, Yu KE, Dussik CM, Kyriakides TR, Lee FY (2021) Locally delivered adjuvant biofilm-penetrating antibiotics rescue impaired endochondral fracture healing caused by MRSA infection. J Orthop Res 39: 402-414. 
Carli AV, Bhimani S, Yang X, Shirley MB, de Mesy Bentley KL, Ross FP, Bostrom MPG (2017) Quantification of peri-implant bacterial load and in vivo biofilm formation in an innovative, clinically representative mouse model of periprosthetic joint infection. J Bone Joint Surg Am 99: e25. DOI: 10.2106/ JBJS.16.00815.

Carli AV, Bhimani S, Yang X, de Mesy Bentley KL, Ross FP, Bostrom MPG (2018) Vancomycinloaded polymethylmethacrylate spacers fail to eradicate periprosthetic joint infection in a clinically representative mouse model. J Bone Joint Surg Am 100: e76. DOI: 10.2106/JBJS.17.01100.

Cassat JE, Hammer ND, Campbell JP, Benson MA, Perrien DS, Mrak LN, Smeltzer MS, Torres VJ, Skaar EP (2013) A secreted bacterial protease tailors the Staphylococcus aureus virulence repertoire to modulate bone remodeling during osteomyelitis. Cell Host Microbe 13: 759-772.

Chadha HS, Fitzgerald RH, Wiater P, Sud S, Nasser S, Wooley PH (1999) Experimental acute hematogenous osteomyelitis in mice. I. Histopathological and immunological findings. J Orthop Res 17: 376-381.

Chen X, Jiao J, He X, Zhang J, Wang H, Xu Y, Jin $\mathrm{T}$ (2017) CHI3L1 regulation of inflammation and the effects on osteogenesis in a Staphylococcus aureus -induced murine model of osteomyelitis. FEBS J 284: 1738-1747.

Choe H, Narayanan AS, Gandhi DA, Weinberg A, Marcus RE, Lee Z, Bonomo RA, Greenfield EM (2015) Immunomodulatory peptide IDR-1018 decreases implant infection and preserves osseointegration. Clin Orthop Relat Res 473: 2898-2907.

Coenye T, Goeres D, Van Bambeke F, Bjarnsholt T (2018) Should standardized susceptibility testing for microbial biofilms be introduced in clinical practice? Clin Microbiol Infect 24: 570-572.

Crabbé A, Jensen PØ, Bjarnsholt T, Coenye T (2019) Antimicrobial tolerance and metabolic adaptations in microbial biofilms. Trends Microbiol 27: 850-863.

Crane DP, Gromov K, Li D, Søballe K, Wahnes C, Büchner H, Hilton MJ, O'Keefe RJ, Murray CK, Schwarz EM (2009) Efficacy of colistin-impregnated beads to prevent multidrug-resistant $A$. baumannii implant-associated osteomyelitis. J Orthop Res 27: 1008-1015.

de Mesy Bentley KL, Trombetta R, Nishitani K, Bello-Irizarry SN, Ninomiya M, Zhang L, Chung HL, McGrath JL, Daiss JL, Awad HA, Kates SL, Schwarz EM (2017) Evidence of Staphylococcus aureus deformation, proliferation, and migration in canaliculi of live cortical bone in murine models of osteomyelitis: S. aureus in canaliculi of cortical bone in murine models of osteomyelitis. J Bone Miner Res 32: 985-990.

Elasri MO, Thomas JR, Skinner RA, Blevins JS, Beenken KE, Nelson CL, Smelter MS (2002) Staphylococcus aureus collagen adhesin contributes to the pathogenesis of osteomyelitis. Bone 30: 275-280.
Ellington JK, Harris M, Hudson MC, Vishin S, Webb LX, Sherertz R (2006) Intracellular Staphylococcus aureus and antibiotic resistance: implications for treatment of staphylococcal osteomyelitis. J Orthop Res 24: 87-93.

Farnsworth CW, Schott EM, Benvie AM, Zukoski J, Kates SL, Schwarz EM, Gill SR, Zuscik MJ, Mooney RA (2018) Obesity/type 2 diabetes increases inflammation, periosteal reactive bone formation, and osteolysis during Staphylococcus aureus implantassociated bone infection. J Orthop Res 36: 1614-1623.

Farnsworth CW, Schott EM, Jensen SE, Zukoski J, Benvie AM, Refaai MA, Kates SL, Schwarz EM, Zuscik MJ, Gill SR, Mooney RA (2017) Adaptive upregulation of clumping factor A (ClfA) by Staphylococcus aureus in the obese, type 2 diabetic host mediates increased virulence. Infect Immun 85: e01005-16. DOI:10.1128/IAI.01005-16.

Farnsworth CW, Shehatou CT, Maynard R, Nishitani K, Kates SL, Zuscik MJ, Schwarz EM, Daiss JL, Mooney RA (2015) A humoral immune defect distinguishes the response to Staphylococcus aureus infections in mice with obesity and type 2 diabetes from that in mice with type 1 diabetes. Infect Immun 83: 2264-2274.

Ferguson VL, Ayers RA, Bateman TA, Simske SJ (2003) Bone development and age-related bone loss in male C57BL/6J mice. Bone 33: 387-398.

Ford CA, Spoonmore TJ, Gupta MK, Duvall CL, Guelcher SA, Cassat JE (2020) Diflunisalloaded poly(propylene sulfide) nanoparticles decrease $S$. aureus-mediated bone destruction during osteomyelitis. J Orthop Res 39: 426-437.

Funao H, Ishii K, Nagai S, Sasaki A, Hoshikawa T, Aizawa M, Okada Y, Chiba K, Koyasu S, Toyama Y, Matsumoto M (2012) Establishment of a real-time, quantitative, and reproducible mouse model of Staphylococcus osteomyelitis using bioluminescence imaging. Infect Immun 80: 733-741.

Funao H, Nagai S, Sasaki A, Hoshikawa T, Tsuji T, Okada Y, Koyasu S, Toyama Y, Nakamura M, Aizawa M, Matsumoto M, Ishii K (2016) A novel hydroxyapatite film coated with ionic silver via inositol hexaphosphate chelation prevents implantassociated infection. Sci Rep 6: 23238. DOI: 10.1038/ srep23238.

Gargiulo S, Greco A, Gramanzini M, Esposito S, Affuso A, Brunetti A, Vesce G (2012) Mice anesthesia, analgesia, and care, part I: anesthetic considerations in preclinical research. ILAR J 53: E55-69.

Gedbjerg N, LaRosa R, Hunter JG, Varrone JJ, Kates SL, Schwarz EM, Daiss JL (2013) Antiglucosaminidase IgG in sera as a biomarker of host immunity against Staphylococcus aureus in orthopaedic surgery patients. J Bone Jointt Surg Am 95: e171. DOI: 10.2106/JBJS.L.01654.

Guggenberger C, Wolz C, Morrissey JA, Heesemann J (2012) Two distinct coagulasedependent barriers protect Staphylococcus aureus from neutrophils in a three dimensional in vitro infection 
model. PLoS Pathog 8: e1002434. DOI: 10.1371/ journal.ppat.1002434.

Haffner-Luntzer M, Kovtun A, Rapp AE, Ignatius A (2016) Mouse models in bone fracture healing research. Curr Mol Biol Rep 2: 101-111.

Harris MA, Beenken KE, Smeltzer MS, Haggard WO, Jennings AJ (2017) Phosphatidylcholine coatings deliver local antimicrobials and reduce infection in a murine model: a preliminary study. Clin Orthop Relat Res 475: 1847-1853.

Hegde V, Dworsky EM, Stavrakis AI, Loftin AH, Zoller SD, Park HY, Richman S, Johansen D, Hu Y, Taylor JA, Hamad CD, Chun RF, Xi W, Adams JS, Bernthal NM (2017) Single-dose, preoperative vitamin-d supplementation decreases infection in a mouse model of periprosthetic joint infection. J Bone Joint Surg Am 99: 1737-1744.

Heim CE, Vidlak D, Kielian T (2015a) Interleukin-10 production by myeloid-derived suppressor cells contributes to bacterial persistence during Staphylococcus aureus orthopedic biofilm infection. J Leukoc Biol 98: 1003-1013.

Heim CE, Vidlak D, Scherr TD, Hartman CW, Garvin KL, Kielian T (2015b) IL-12 promotes myeloidderived suppressor cell recruitment and bacterial persistence during Staphylococcus aureus orthopedic implant infection. J Immunol 194: 3861-3872.

Heim CE, Vidlak D, Scherr TD, Kozel JA, Holzapfel M, Muirhead DE, Kielian T (2014) Myeloidderived suppressor cells contribute to Staphylococcus aureus orthopedic biofilm infection. J Immunol 192: 3778-3792.

Heim CE, West SC, Ali H, Kielian T (2018) Heterogeneity of Ly6 $\mathrm{G}^{+} \mathrm{Ly}_{6 \mathrm{C}^{+}}$myeloid-derived suppressor cell infiltrates during Staphylococcus aureus biofilm infection. Infect Immun 86: e00684-18. DOI: 10.1128/IAI.00684-18.

Hernandez CJ, Yang X, Ji G, Niu Y, Sethuraman AS, Koressel J, Shirley M, Fields MW, Chyou S, Li TM, Luna M, Callahan RL, Ross FP, Lu TT, Brito IL, Carli AV, Bostrom MPG (2019) Disruption of the gut microbiome increases the risk of periprosthetic joint infection in mice. Clin Orthop Relat Res 477: 25882598.

Histing T, Garcia P, Holstein JH, Klein M, Matthys R, Nuetzi R, Steck R, Laschke MW, Wehner T, Bindl R, Recknagel S, Stuermer EK, Vollmar B, Wildemann B, Lienau J, Willie B, Peters A, Ignatius A, Pohlemann T, Claes L, Menger MD (2011) Small animal bone healing models: standards, tips, and pitfalls results of a consensus meeting. Bone 49: 591-599.

Hofstee MI, Riool M, Terjajevs I, Thompson K, Stoddart MJ, Richards RG, Zaat SAJ, Moriarty TF (2020) Three-dimensional in vitro Staphylococcus aureus abscess communities display antibiotic tolerance and protection from neutrophil clearance. Infect Immun 88: e00293-20. DOI: 10.1128/IAI.0029320.

Holstein JH, Garcia P, Histing T, Kristen A, Scheuer C, Menger MD, Pohlemann T (2009) Advances in the establishment of defined mouse models for the study of fracture healing and bone regeneration. J Orthop Trauma 23: S31-S38.

Horst SA, Hoerr V, Beineke A, Kreis C, Tuchscherr L, Kalinka J, Lehne S, Schleicher I, Köhler G, Fuchs T, Raschke MJ, Rohde M, Peters G, Faber C, Löffler B, Medina E (2012) A novel mouse model of Staphylococcus aureus chronic osteomyelitis that closely mimics the human infection. Am J Pathol 181: 1206-1214.

Hou Y, Qin H, Jiang N, Liu G, Wu H, Bai L, Yu B, Zhang X (2019) G-CSF partially mediates bone loss induced by Staphylococcus aureus infection in mice. Clin Sci 133: 1297-1308.

Inzana JA, Trombetta R, Schwarz EM, Kates SL, Awad HA (2015a) 3D printed bioceramics for dual antibiotic delivery to treat implant-associated bone infection. Eur Cell Mater 30: 232-247.

Inzana JA, Schwarz EM, Kates SL, Awad HA (2015b) A novel murine model of established Staphylococcal bone infection in the presence of a fracture fixation plate to study therapies utilizing antibiotic-laden spacers after revision surgery. Bone 72: 128-136.

Ishikawa M, de Mesy Bentley KL, McEntire BJ, Bal BS, Schwarz EM, Xie C (2017) Surface topography of silicon nitride affects antimicrobial and osseointegrative properties of tibial implants in a murine model. J Biomed Mater Res A 105: 3413-3421.

Isogai N, Shiono Y, Kuramoto T, Yoshioka K, Ishihama $\mathrm{H}$, Funao $\mathrm{H}$, Nakamura $\mathrm{M}$, Matsumoto M, Ishii K (2020) Potential osteomyelitis biomarkers identified by plasma metabolome analysis in mice. Sci Rep 10: 839. DOI:10.1038/s41598020-57619-1.

Ji Z, Su J, Hou Y, Yao Z, Yu B, Zhang X (2020) EGFR/FAK and C-SRC signalling pathways mediate the internalisation of Stapylococcus aureus by osteoblasts. Cell Microbiol 22: e13240. DOI: 10.1111/ cmi.13240.

Jiang $\mathrm{Y}$, Wang $\mathrm{S}, \mathrm{Wu} \mathrm{H}$, Qin $\mathrm{H}$, Ren $\mathrm{M}$, Lin J, Yu B (2019) Aspirin alleviates orthopedic implant-associated infection. Int J Mol Med 44: 12811288.

Jilka RL (2013) The relevance of mouse models for investigating age-related bone loss in humans. J Gerontol A Biol Sci Med Sci 68: 1209-1217.

Johansson A, Flock JI, Svensson O (2001) Collagen and fibronectin binding in experimental staphylococcal osteomyelitis. Clin Orthop Relat Res 382: 241-246.

Johnson CT, Wroe JA, Agarwal R, Martin KE, Guldberg RE, Donlan RM, Westblade LF, García AJ (2018) Hydrogel delivery of lysostaphin eliminates orthopedic implant infection by Staphylococcus aureus and supports fracture healing. Proc Natl Acad Sci 115: E4960-E4969.

Jørgensen NP, Hansen K, Andreasen C, Pedersen M, Fuursted K, Meyer R, Petersen E (2017) Hyperbaric oxygen therapy is ineffective as an adjuvant to daptomycin with rifampicin treatment in a murine model of Staphylococcus aureus in implant-associated 
osteomyelitis. Microorganisms 5: 21. DOI: 10.3390/ microorganisms5020021.

Jørgensen NP, Meyer R, Dagnæs-Hansen F, Fuursted K, Petersen E (2014) A modified chronic infection model for testing treatment of Staphylococcus aureus biofilms on implants. PLoS One 9: e103688. DOI: 10.1371/journal.pone.0103688.

Jørgensen NP, Skovdal SM, Meyer RL, DagnæsHansen F, Fuursted K, Petersen E (2016) Rifampicincontaining combinations are superior to combinations of vancomycin, linezolid and daptomycin against Staphylococcus aureus biofilm infection in vivo and in vitro. Pathog Dis 74: ftw019. DOI: 10.1093/femspd/ ftw019.

Kaur S, Harjai K, Chhibber S (2016) In vivo assessment of phage and linezolid based implant coatings for treatment of methicillin resistant $S$. aureus (MRSA) mediated orthopaedic device related infections. PLoS One 11: e0157626. DOI: 10.1371/ journal.pone.0157626.

Kavanagh N, Ryan EJ, Widaa A, Sexton G, Fennell J, O'Rourke S, Cahill KC, Kearney CJ, O'Brien FJ, Kerrigan SW (2018) Staphylococcal osteomyelitis: disease progression, treatment challenges, and future directions. Clin Microbiol Rev 31: e00084-17. DOI: 10.1128/CMR.00084-17.

Kohler T, Beyeler M, Webster D, Müller R (2005) Compartmental bone morphometry in the mouse femur: reproducibility and resolution dependence of microtomographic measurements. Calcif Tissue Int 77: 281-290. DOI: 10.1007/s00223-005-0039-2.

Laperre K, Depypere M, van Gastel N, Torrekens S, Moermans K, Bogaerts R, Maes F, Carmeliet G (2011) Development of micro-CT protocols for in vivo follow-up of mouse bone architecture without major radiation side effects. Bone 49: 613-622.

Lazzarini L, Overgaard KA, Conti E, Shirtliff ME (2006) Experimental osteomyelitis: what have we learned from animal studies about the systemic treatment of osteomyelitis? J Chemother 18: 451-460.

Lee LY, Miyamoto YJ, McIntyre BW, Höök M, McCrea KW, McDevitt D, Brown EL (2002) The Staphylococcus aureus map protein is an immunomodulator that interferes with $\mathrm{T}$ cellmediated responses. J Clin Invest 110: 1461-1471.

Letson HL, Morris J, Biros E, Dobson GP (2019) Conventional and specific-pathogen free rats respond differently to anesthesia and surgical trauma. Sci Rep 9: 9399. DOI: 10.1038/s41598-019-45871-z.

Lew DP, Waldvogel FA (2004) Osteomyelitis. Lancet 364: 369-379.

Li D, Gromov K, Søballe K, Puzas JE, O'Keefe RJ, Awad H, Drissi H, Schwarz EM (2008) Quantitative mouse model of implant-associated osteomyelitis and the kinetics of microbial growth, osteolysis, and humoral immunity. J Orthop Res 26: 96-105.

Lin Y, Su J, Wang Y, Xu D, Zhang X, Yu B (2021) mRNA transcriptome analysis of bone in a mouse model of implant-associated Staphylococcus aureus osteomyelitis. Infect Immun 89: e00814-20. DOI: 10.1128/IAI.00814-20.
Lindsay SE, Lindsay HG, Kallet J, Weaver MR, Curran-Everett D, Crapo JD, Regan EA (2021) MnTE-2-PyP disrupts Staphylococcus aureus biofilms in a novel fracture model. J Orthop Res. DOI: 10.1002/ jor.24967.

Loughran AJ, Gaddy D, Beenken KE, Meeker DG, Morello R, Zhao H, Byrum SD, Tackett AJ, Cassat JE, Smeltzer MS (2016) Impact of sar A and phenol-soluble modulins on the pathogenesis of osteomyelitis in diverse clinical isolates of Staphylococcus aureus. Infect Immun 84: 2586-2594.

Lovati AB, Drago L, Monti L, De Vecchi E, Previdi S, Banfi G, Romanò CL (2013) Diabetic mouse model of orthopaedic implant-related Staphylococcus aureus infection. PLoS One 8: e67628. DOI: 10.1371/journal. pone.0067628.

Lovati AB, Romanò CL, Monti L, Vassena C, Previdi S, Drago L (2014) Does PGE1 vasodilator prevent orthopaedic implant-related infection in diabetes? Preliminary results in a mouse model. PLoS One 9: e94758. DOI: 10.1371/journal.pone.0094758.

Lu X, Chen R, Lv J, Xu W, Chen H, Ma Z, Huang S, Li S, Liu H, Hu J, Nie L (2019) High-resolution bimodal imaging and potent antibiotic/photodynamic synergistic therapy for osteomyelitis with a bacterial inflammation-specific versatile agent. Acta Biomater 99: 363-372.

Lüthje FL, Jensen LK, Jensen HE, Skovgaard K (2020) The inflammatory response to bone infection - a review based on animal models and human patients. APMIS 128: 275-286.

Mandell JB, Deslouches B, Montelaro RC, Shanks RMQ, Doi Y, Urish KL (2017) Elimination of antibiotic resistant surgical implant biofilms using an engineered cationic amphipathic peptide WLBU2. Sci Rep 7: 18098. DOI: 10.1038/s41598-017-17780-6.

Marriott I, Gray DL, Rati DM, Fowler VG, Stryjewski ME, Levin LS, Hudson MC, Bost KL (2005) Osteoblasts produce monocyte chemoattractant protein-1 in a murine model of Staphylococcus aureus osteomyelitis and infected human bone tissue. Bone 37: 504-512.

Marriott I, Gray DL, Tranguch SL, Fowler VG, Stryjewski M, Scott Levin L, Hudson MC, Bost KL (2004) Osteoblasts express the inflammatory cytokine interleukin-6 in a murine model of Staphylococcus aureus osteomyelitis and infected human bone tissue. Am J Pathol 164: 1399-1406.

Masters EA, Hao SP, Kenney HM, Morita Y, Galloway CA, Mesy Bentley KL, Ricciardi BF, Boyce BF, Schwarz EM, Oh I (2021) Distinct vasculotropic versus osteotropic features of $S$. agalactiae versus $S$. aureus implant-associated bone infection in mice. J Orthop Res 39: 389-401.

Masters EA, de Mesy Bentley KL, Gill AL, Hao SP, Galloway CA, Salminen AT, Guy DR, McGrath JL, Awad HA, Gill SR, Schwarz EM (2020) Identification of penicillin binding protein 4 (PBP4) as a critical factor for Staphylococcus aureus bone invasion during osteomyelitis in mice. PLoS Pathog 16: e1008988. 
Masters EA, Trombetta RP, de Mesy Bentley KL, Boyce BF, Gill AL, Gill SR, Nishitani K, Ishikawa $\mathrm{M}$, Morita $\mathrm{Y}$, Ito $\mathrm{H}$, Bello-Irizarry $\mathrm{SN}$, Ninomiya M, Brodell JD, Lee CC, Hao SP, Oh I, Xie C, Awad HA, Daiss JL, Owen JR, Kates SL, Schwarz EM, Muthukrishnan G (2019) Evolving concepts in bone infection: redefining "biofilm", "acute vs. chronic osteomyelitis", "the immune proteome" and "local antibiotic therapy". Bone Res 7: 20. DOI: 10.1038/ s41413-019-0061-z.

Mendoza Bertelli A, Delpino MV, Lattar S, Giai C, Llana MN, Sanjuan N, Cassat JE, Sordelli D, Gómez MI (2016) Staphylococcus aureus protein A enhances osteoclastogenesis via TNFR1 and EGFR signaling. Biochim Biophys Acta 1862: 1975-1983.

Metsemakers WJ, Emanuel N, Cohen O, Reichart M, Potapova I, Schmid T, Segal D, Riool M, Kwakman PHS, de Boer L, de Breij A, Nibbering PH, Richards RG, Zaat SAJ, Moriarty TF (2015) A doxycyclineloaded polymer-lipid encapsulation matrix coating for the prevention of implant-related osteomyelitis due to doxycycline-resistant methicillin-resistant Staphylococcus aureus. J Control Release 209: 47-56.

Mills LA, Simpson AHRW (2012) In vivo models of bone repair. J Bone Joint Surg Br 94: 865-874.

Moriarty TF, Harris LG, Mooney RA, Wenke JC, Riool M, Zaat SAJ, Moter A, Schaer TP, Khanna N, Kuehl R, Alt V, Montali A, Liu J, Zeiter S, Busscher HJ, Grainger DW, Richards RG (2019) Recommendations for design and conduct of preclinical in vivo studies of orthopedic device-related infection. J Orthop Res 37: 271-287.

Moriarty TF, Kuehl R, Coenye T, Metsemakers WJ, Morgenstern M, Schwarz EM, Riool M, Zaat SAJ, Khana N, Kates SL, Richards RG (2016) Orthopaedic device-related infection: current and future interventions for improved prevention and treatment. EFORT Open Rev 1: 89-99.

Muschler GF, Raut VP, Patterson TE, Wenke JC, Hollinger JO (2010) The design and use of animal models for translational research in bone tissue engineering and regenerative medicine. Tissue Eng Part B Rev 16: 123-145.

Nishitani K, Beck CA, Rosenberg AF, Kates SL, Schwarz EM, Daiss JL (2015a) A diagnostic serum antibody test for patients with Staphylococcus aureus osteomyelitis. Clin Orthop Relat Res 473: 2735-2749.

Nishitani K, Sutipornpalangkul W, de Mesy Bentley KL, Varrone JJ, Bello-Irizarry SN, Ito H, Matsuda S, Kates SL, Daiss JL, Schwarz EM (2015b) Quantifying the natural history of biofilm formation in vivo during the establishment of chronic implantassociated Staphylococcus aureus osteomyelitis in mice to identify critical pathogen and host factors. J Orthop Res 33: 1311-1319.

Niska JA, Meganck JA, Pribaz JR, Shahbazian JH, Lim E, Zhang N, Rice BW, Akin A, Ramos RI, Bernthal NM, Francis KP, Miller LS (2012a) Monitoring bacterial burden, inflammation and bone damage longitudinally using optical and $\mu \mathrm{CT}$ imaging in an orthopaedic implant infection in mice. PLoS One 7: e47397. DOI: 10.1371/journal. pone.0047397.

Niska JA, Shahbazian JH, Ramos RI, Francis KP, Bernthal NM, Miller LS (2013) Vancomycin-rifampin combination therapy has enhanced efficacy against an experimental Staphylococcus aureus prosthetic joint infection. Antimicrob Agents Chemother 57: 50805086.

Niska JA, Shahbazian JH, Ramos RI, Pribaz JR, Billi F, Francis KP, Miller LS (2012b) Daptomycin and tigecycline have broader effective dose ranges than vancomycin as prophylaxis against a Staphylococcus aureus surgical implant infection in mice. Antimicrob Agents Chemother 56: 2590-2597.

Oezel L, Büren C, Scholz AO, Windolf J, Windolf CD (2019) Effect of antibiotic infused calcium sulfate/hydroxyapatite (CAS/HA) insets on implantassociated osteitis in a femur fracture model in mice. PLoS One 14: e0213590. DOI: 10.1371/journal. pone.0213590.

O'Neill J (2014) Antimicrobial resistance. Tackling a crisis for the health and wealth of nations. Review on Antimicrobial Resistance. Wellcome Collection, London.

Patel M, Rojavin Y, Jamali A, Wasielewski S, Salgado C (2009) Animal models for the study of osteomyelitis. Semin Plast Surg 23: 148-154.

Pearce AI, Richards RG, Milz S, Schneider E, Pearce SG (2007) Animal models for implant biomaterial research in bone: a review. Eur Cell Mater 13: $1-10$.

Peng KT, Chiang YC, Huang TY, Chen PC, Chang PJ, Lee CW (2019) Curcumin nanoparticles are a promising anti-bacterial and anti-inflammatory agent for treating periprosthetic joint infections. Int J Nanomedicine 14: 469-481.

Peng KT, Hsieh CC, Huang TY, Chen PC, Shih HN, Lee MS, Chang PJ (2017) Staphylococcus aureus biofilm elicits the expansion, activation and polarization of myeloid-derived suppressor cells in vivo and in vitro. PLoS One 12: e0183271. DOI: 10.1371/journal. pone. 0183271.

Pickett JE, Thompson JM, Sadowska A, Tkaczyk C, Sellman BR, Minola A, Corti D, Lanzavecchia A, Miller LS, Thorek DL (2018) Molecularly specific detection of bacterial lipoteichoic acid for diagnosis of prosthetic joint infection of the bone. Bone Res 6 : 13. DOI: 10.1038/s41413-018-0014-y.

Popescu B, Tevanov I, Carp M, Ulici A (2020) Acute hematogenous osteomyelitis in pediatric patients: epidemiology and risk factors of a poor outcome. J Int Med Res 48: 0300060520910889. DOI: 10.1177/0300060520910889.

Potter AD, Butrico CE, Ford CA, Curry JM, Trenary IA, Tummarakota SS, Hendrix AS, Young JD, Cassat JE (2020) Host nutrient milieu drives an essential role for aspartate biosynthesis during invasive Staphylococcus aureus infection. Proc Natl Acad Sci U S A 117: 12394-12401.

Prabhakara R, Harro JM, Leid JG, Harris M, Shirtliff ME (2011a) Murine immune response to a 
chronic Staphylococcus aureus biofilm infection. Infect Immun 79: 1789-1796.

Prabhakara R, Harro JM, Leid JG, Keegan AD, Prior ML, Shirtliff ME (2011b) Suppression of the inflammatory immune response prevents the development of chronic biofilm infection due to methicillin-resistant Staphylococcus aureus. Infect Immun 79: 5010-5018.

Pribaz JR, Bernthal NM, Billi F, Cho JS, Ramos RI, Guo Y, Cheung AL, Francis KP, Miller LS (2012) Mouse model of chronic post-arthroplasty infection: noninvasive in vivo bioluminescence imaging to monitor bacterial burden for long-term study. J Orthop Res 30: 335-340.

Putnam NE, Fulbright LE, Curry JM, Ford CA, Petronglo JR, Hendrix AS, Cassat JE (2019) MyD88 and IL-1R signaling drive antibacterial immunity and osteoclast-driven bone loss during Staphylococcus aureus osteomyelitis. PLoS Pathog 15: e1007744. DOI: 10.1371/journal.ppat.1007744.

Qadri S, Haik Y, Mensah-Brown E, Bashir G, Fernandez-Cabezudo MJ, al-Ramadi BK (2017) Metallic nanoparticles to eradicate bacterial bone infection. Nanomedicine 13: 2241-2250.

Ramirez AM, Byrum SD, Beenken KE, Washam C, Edmondson RD, Mackintosh SG, Spencer HJ, Tackett AJ, Smeltzer MS (2020) Exploiting correlations between protein abundance and the functional status of saeRS and sarA to identify virulence factors of potential importance in the pathogenesis of Staphylococcus aureus osteomyelitis. ACS Infect Dis 6: 237-249.

Reizner W, Hunter J, O’Malley N, Southgate R, Schwarz E, Kates S (2014) A systematic review of animal models for Staphylococcus aureus osteomyelitis. Eur Cell Mater 27: 196-212.

Rochford ETJ, Sabaté Brescó M, Poulsson AHC, Kluge K, Zeiter S, Ziegler M, O’Mahony L, Richards RG, Moriarty TF (2019) Infection burden and immunological responses are equivalent for polymeric and metallic implant materials in vitro and in a murine model of fracture-related infection. J Biomed Mater Res B Appl Biomater 107: 1095-1106.

Rochford ETJ, Sabaté Brescó M, Zeiter S, Kluge K, Poulsson A, Ziegler M, Richards RG, O'Mahony L, Moriarty TF (2016) Monitoring immune responses in a mouse model of fracture fixation with and without Staphylococcus aureus osteomyelitis. Bone 83: 82-92.

Rom JS, Ramirez AM, Beenken KE, Sahukhal GS, Elasri MO, Smeltzer MS (2020) The Impacts of $m s a A B C R$ on $s a r A$-associated phenotypes are different in divergent clinical isolates of Staphylococcus aureus. Infect Immun 88: e00530-19. DOI: 10.1128/ IAI.00530-19.

Röntgen V, Blakytny R, Matthys R, Landauer M, Wehner T, Göckelmann M, Jermendy P, Amling M, Schinke T, Claes L, Ignatius A (2010) Fracture healing in mice under controlled rigid and flexible conditions using an adjustable external fixator. J Orthop Res 28: 1456-1462.
Sabaté Brescó M, O’Mahony L, Zeiter S, Kluge K, Ziegler M, Berset C, Nehrbass D, Richards RG, Moriarty TF (2017) Influence of fracture stability on Staphylococcus epidermidis and Staphylococcus aureus infection in a murine femoral fracture model. Eur Cell Mater 34: 321-340.

Sander G, Börner T, Kriegeskorte A, von Eiff C, Becker K, Mahabir E (2012) Catheter colonization and abscess formation due to Staphylococcus epidermidis with normal and small-colony-variant phenotype is mouse strain dependent. PLoS One 7: e36602. DOI: 10.1371/journal.pone.0036602.

Scherr TD, Hanke ML, Huang O, James DBA, Horswill AR, Bayles KW, Fey PD, Torres VJ, Kielian $\mathrm{T}$ (2015) Staphylococcus aureus biofilms induce macrophage dysfunction through leukocidin AB and alpha-toxin. mBio 6: e01021-15. DOI: 10.1128/ mBio.01021-15.

Sellers RS, Clifford CB, Treuting PM, Brayton C (2012) Immunological variation between inbred laboratory mouse strains: points to consider in phenotyping genetically immunomodified mice. Vet Pathol 49: 32-43.

Shandley S, Matthews KP, Cox J, Romano D, Abplanalp A, Kalns J (2012) Hyperbaric oxygen therapy in a mouse model of implant-associated osteomyelitis. J Orthop Res 30: 203-208.

Sheppard WL, Mosich GM, Smith RA, Hamad CD, Park HY, Zoller SD, Trikha R, McCoy TK, Borthwell R, Hoang J, Truong N, Cevallos N, Clarkson S, Hori KR, van Dijl JM, Francis KP, Petrigliano FA, Bernthal NM (2020) Novel in vivo mouse model of shoulder implant infection. J Shoulder Elbow Surg 29: 14121424.

Shiono Y, Ishii K, Nagai S, Kakinuma H, Sasaki A, Funao H, Kuramoto T, Yoshioka K, Ishihama H, Isogai N, Takeshima K, Tsuji T, Okada Y, Koyasu S, Nakamura M, Toyama Y, Aizawa M, Matsumoto M (2016) Delayed propionibacterium acnes surgical site infections occur only in the presence of an implant. Sci Rep 6: 32758. DOI: 10.1038/srep32758.

Sosa BR, Niu Y, Turajane K, Staats K, Suhardi V, Carli A, Fischetti V, Bostrom M, Yang X (2020) 2020 John Charnley Award: the antimicrobial potential of bacteriophage-derived lysin in a murine debridement, antibiotics, and implant retention model of prosthetic joint infection. Bone Joint J 102-B: 3-10.

Sottnik JL, U'Ren LW, Thamm DH, Withrow SJ, Dow SW (2010) Chronic bacterial osteomyelitis suppression of tumor growth requires innate immune responses. Cancer Immunol Immunother 59: 367-378.

Spoonmore TJ, Ford CA, Curry JM, Guelcher SA, Cassat JE (2020) Concurrent local delivery of diflunisal limits bone destruction but fails to improve systemic vancomycin efficacy during Staphylococcus aureus osteomyelitis. Antimicrob Agents Chemother 64: e00182-20. DOI: 10.1128/AAC.00182-20.

Stavrakis AI, Zhu S, Loftin AH, Weixian X, Niska J, Hegde V, Segura T, Bernthal NM (2019) Controlled release of vancomycin and tigecycline 
from an orthopaedic implant coating prevents Staphylococcus aureus infection in an open fracture animal model. Biomed Res Int 2019: 1638508. DOI: 10.1155/2019/1638508.

Stavrakis AI, Zhu S, Hegde V, Loftin AH, Ashbaugh AG, Niska JA, Miller LS, Segura T, Bernthal NM (2016) In vivo efficacy of a "smart" antimicrobial implant coating: J Bone Joint Surg Am 98: 1183-1189.

Szafranska AK, Oxley APA, Chaves-Moreno D, Horst SA, Roßlenbroich S, Peters G, Goldmann O, Rohde M, Sinha B, Pieper DH, Löffler B, Jauregui R, Wos-Oxley ML, Medina E (2014) High-resolution transcriptomic analysis of the adaptive response of Staphylococcus aureus during acute and chronic phases of osteomyelitis. mBio 5: e01775-14. DOI: 10.1128/ mBio.01775-14.

Takahashi T, Yokogawa K, Sakura N, Nomura M, Kobayashi S, Miyamoto K (2008) Bone-targeting of quinolones conjugated with an acidic oligopeptide. Pharm Res 25: 2881-2888.

Takao K, Miyakawa T (2015) Genomic responses in mouse models greatly mimic human inflammatory diseases. Proc Natl Acad Sci U S A 112: 1167-1172.

Thompson JM, Miller RJ, Ashbaugh AG, Dillen CA, Pickett JE, Wang Y, Ortines RV, Sterling RS, Francis KP, Bernthal NM, Cohen TS, Tkaczyk C, Yu L, Stover CK, DiGiandomenico A, Sellman BR, Thorek DLJ, Miller LS (2018) Mouse model of Gram-negative prosthetic joint infection reveals therapeutic targets. JCI Insight 3: e121737. DOI: 10.1172/jci.insight.121737.

Thompson JM, Saini V, Ashbaugh AG, Miller RJ, Ordonez AA, Ortines RV, Wang Y, Sterling RS, Jain SK, Miller LS (2017) Oral-only linezolid-rifampin is highly effective compared with other antibiotics for periprosthetic joint infection: study of a mouse model. J Bone Joint Surg Am 99: 656-665.

Tomizawa T, Ishikawa M, Bello-Irizarry SN, Mesy Bentley KL, Ito H, Kates SL, Daiss JL, Beck C, Matsuda S, Schwarz EM, Nishitani K (2020) Biofilm producing Staphylococcus epidermidis (RP62A strain) inhibits osseous integration without osteolysis and histopathology in a murine septic implant model. J Orthop Res 38: 852-860.

Trombetta RP, Ninomiya MJ, El-Atawneh IM, Knapp EK, de Mesy Bentley KL, Dunman PM, Schwarz EM, Kates SL, Awad HA (2019a) Calcium phosphate spacers for the local delivery of sitafloxacin and rifampin to treat orthopedic infections: efficacy and proof of concept in a mouse model of single-stage revision of device-associated osteomyelitis. Pharmaceutics 11: 94. DOI: 10.3390/ pharmaceutics11020094.

Trombetta RP, de Mesy Bentley KL, Schwarz EM, Kate SL, Awad HA (2019b) A murine femoral ostectomy model with hardware exchange to assess antibiotic-impregnated spacers for implantassociated osteomyelitis. Eur Cell Mater 37: 431-444.

Tuchscherr L, Geraci J, Löffler B (2017) Staphylococcus aureus regulator sigma B is important to develop chronic infections in hematogenous murine osteomyelitis model. Pathogens 6: 31. DOI: 10.3390/pathogens6030031.

Tuchscherr L, Kreis CA, Hoerr V, Flint L, Hachmeister M, Geraci J, Bremer-Streck S, Kiehntopf M, Medina E, Kribus M, Raschke M, Pletz M, Peters G, Löffler B (2016) Staphylococcus aureus develops increased resistance to antibiotics by forming dynamic small colony variants during chronic osteomyelitis. J Antimicrob Chemother 71: 438-448.

Tuchscherr L, Pöllath C, Siegmund A, DeinhardtEmmer S, Hoerr V, Svensson CM, Thilo Figge M, Monecke S, Löffler B (2019) Clinical S. aureus isolates vary in their virulence to promote adaptation to the host. Toxins 11: 135. DOI: 10.3390/toxins11030135.

Tuohy JL, Fogle JE, Meichner K, Borst LB, Petty CS, Griffith EH, Osborne JA, Lascelles BDX (2018) Assessment of a novel nanoparticle hyperthermia therapy in a murine model of osteosarcoma. Vet Surg 47: 1021-1030.

Valour F, Trouillet-Assant S, Riffard N, Tasse J, Flammier S, Rasigade JP, Chidiac C, Vandenesch F, Ferry T, Laurent F (2015) Antimicrobial activity against intraosteoblastic Staphylococcus aureus. Antimicrob Agents Chemother 59: 2029-2036.

Varoga D, Tohidnezhad M, Paulsen F, Wruck CJ, Brandenburg L, Mentlein R, Lippross S, Hassenpflug J, Besch L, Müller M, Jürgens C, Seekamp A, Schmitt L, Pufe T (2008) The role of human beta-defensin-2 in bone. J Anat 213: 749-757.

Varoga D, Wruck CJ, Tohidnezhad M, Brandenburg L, Paulsen F, Mentlein R, Seekamp A, Besch L, Pufe T (2009) Osteoblasts participate in the innate immunity of the bone by producing human beta defensin-3. Histochem Cell Biol 131: 207-218.

Varrone JJ, de Mesy Bentley KL, Bello-Irizarry SN, Nishitani K, Mack S, Hunter JG, Kates SL, Daiss JL, Schwarz EM (2014) Passive immunization with anti-glucosaminidase monoclonal antibodies protects mice from implant-associated osteomyelitis by mediating opsonophagocytosis of Staphylococcus aureus megaclusters. J Orthop Res 32: 1389-1396.

Vidlak D, Kielian T (2016) Infectious dose dictates the host response during Staphylococcus aureus orthopedic-implant biofilm infection. Infect Immun 84: 1957-1965.

Vlasova AN, Takanashi S, Miyazaki A, Rajashekara G, Saif LJ (2019) How the gut microbiome regulates host immune responses to viral vaccines. Curr Opin Virol 37: 16-25.

Wagner JM, Jaurich H, Wallner C, Abraham S, Becerikli M, Dadras M, Harati K, Duhan V, Khairnar V, Lehnhardt M, Behr B (2017) Diminished bone regeneration after debridement of posttraumatic osteomyelitis is accompanied by altered cytokine levels, elevated B cell activity, and increased osteoclast activity. J Orthop Res 35: 2425-2434.

Wagner JM, Reinkemeier F, Dadras M, Wallner C, Huber J, Sogorski A, Sacher M, Schmidt S, Drysch M, Dittfeld S, Becerikli M, Becker K, Rauch N, Lehnhardt 
M, Behr B (2020) Local Wnt3a treatment restores bone regeneration in large osseous defects after surgical debridement of osteomyelitis. J Mol Med 98: 897-906.

Wagner JM, Reinkemeier F, Wallner C, Dadras M, Huber J, Schmidt SV, Drysch M, Dittfeld S, Jaurich H, Becerikli M, Becker K, Rauch N, Duhan V, Lehnhardt M, Behr B (2019) Adipose-derived stromal cells are capable of restoring bone regeneration after post-traumatic osteomyelitis and modulate B-cell response. Stem Cells Transl Med 8: 1084-1091.

Wagner JM, Zöllner H, Wallner C, Ismer B, Schira J, Abraham S, Harati K, Lehnhardt M, Behr B (2016) Surgical debridement is superior to sole antibiotic therapy in a novel murine posttraumatic osteomyelitis model. PLoS One 11: e0149389. DOI: 10.1371/journal.pone.0149389.

Walton KD, Lord A, Kendall LV, Dow SW (2014) Comparison of 3 real-time, quantitative murine models of staphylococcal biofilm infection by using in vivo bioluminescent imaging. Comp Med 64: 25-33.

Wancket LM (2015) Animal models for evaluation of bone implants and devices: comparative bone structure and common model uses. Vet Pathol 52: 842-850.

Wang Y, Thompson JM, Ashbaugh AG, Khodakivskyi P, Budin G, Sinisi R, Heinmiller A, van Oosten M, van Dijl JM, van Dam GM, Francis KP, Bernthal NM, Dubikovskaya EA, Miller LS (2017a) Preclinical evaluation of photoacoustic imaging as a novel noninvasive approach to detect an orthopaedic implant infection. J Am Acad Orthop Surg 25 Suppl 1: S7-S12.

Wang Y, Wang J, Deng Z, Jin J, Jiang H, Meng J, Xu H, Zhao J, Sun G, Qian H (2017b) Chronic osteomyelitis increases the incidence of type 2 diabetes in humans and mice. Int J Biol Sci 13: 11921202.

Wang Y, Ashbaugh AG, Dikeman DA, Zhang J, Ackerman NE, Kim SE, Falgons C, Ortines RV, Liu H, Joyce DP, Alphonse MP, Dillen CA, Thompson JM, Archer NK, Miller LS (2020a) Interleukin-1 $\beta$ and tumor necrosis factor are essential in controlling an experimental orthopedic implant-associated infection. J Orthop Res 38: 1800-1809.

Wang Y, Cheng LI, Helfer DR, Ashbaugh AG, Miller RJ, Tzomides AJ, Thompson JM, Ortines RV, Tsai AS, Liu H, Dillen CA, Archer NK, Cohen TS, Tkaczyk C, Stover CK, Sellman BR, Miller LS (2017c) Mouse model of hematogenous implantrelated Staphylococcus aureus biofilm infection reveals therapeutic targets. Proc Natl Acad Sci U S A 114: E5094-E5102.

Wang Y, Dikeman D, Zhang J, Ackerman N, Kim S, Alphonse MP, Ortines RV, Liu H, Joyce DP, Dillen CA, Thompson JM, Thomas AA, Plaut RD, Miller LS, Archer NK (2021) CCR2 contributes to host defense against Staphylococcus aureus orthopedic implantassociated infections in mice. J Orthop Res. DOI: 10.1002/jor.25027.

Wang $Y$, Lin $Y$, Cheng C, Chen P, Zhang P, Wu H, Li K, Deng Y, Qian J, Zhang X, Yu B (2020b) NF- $\kappa \mathrm{B} / \mathrm{TWIST} 1$ mediates migration and phagocytosis of macrophages in the mice model of implant-associated Staphylococcus aureus osteomyelitis. Front Microbiol 11: 1301. DOI: 10.3389/fmicb.2020.01301.

Mouse Genome Sequencing Consortium, Waterston RH, Lindblad-Toh K, Birney E, Rogers J, Abril JF, Agarwal P, Agarwala R, Ainscough R, Alexandersson M, An P, Antonarakis SE, Attwood J, Baertsch R, Bailey J, Barlow K, Beck S, Berry E, Birren B, Bloom T, Bork P, Botcherby M, Bray N, Brent MR, Brown DG, Brown SD, Bult C, Burton J, Butler J, Campbell RD, Carninci P, Cawley S, Chiaromonte F, Chinwalla T, Church DM, Clamp M, Clee C, Collins FS, Cook LL, Copley RR, Coulson A, Couronne O, Cuff J, Curwen V, Cutts T, Daly M, David R, Davies J, Delehaunty KD, Deri J, Dermitzakis ET, Dewey C, Dickens NJ, Diekhans M, Dodge S, Dubchak I, Dunn DM, Eddy SR, Elnitski L, Emes RD, Eswara P, Eyras E, Felsenfeld A, Fewell GA, Flicek P, Foley K, Frankel WN, Fulton LA, Fulton RS, Furey TS, Gage D, Gibbs RA, Glusman G, Gnerre S, Goldman N, Goodstadt L, Grafham D, Graves TA, Green ED, Gregory S, Guigó R, Guyer M, Hardison RC, Haussler D, Hayashizaki Y, Hillier LW, Hinrichs A, Hlavina W, Holzer T, Hsu F, Hua A, Hubbard T, Hunt A, Jackson I, Jaffe DB, Johnson LS, Jones M, Jones TA, Joy A, Kamal M, Karlsson EK, Karolchik D, Kasprzyk A, Kawai J, Keibler E, Kells C, Kent WJ, Kirby A, Kolbe DL, Korf I, Kucherlapati RS, Kulbokas EJ, Kulp D, Landers $\mathrm{T}$, Leger JP, Leonard S, Letunic I, Levine R, Li J, Li M, Lloyd C, Lucas S, Ma B, Maglott DR, Mardis ER, Matthews L, Mauceli E, Mayer JH, McCarthy M, McCombie WR, McLaren S, McLay K, McPherson JD, Meldrim J, Meredith B, Mesirov JP, Miller W, Miner TL, Mongin E, Montgomery KT, Morgan M, Mott R, Mullikin JC, Muzny DM, Nash WE, Nelson JO, Nhan MN, Nicol R, Ning Z, Nusbaum C, O'Connor MJ, Okazaki Y, Oliver K, Overton-Larty E, Pachter L, Parra G, Pepin KH, Peterson J, Pevzner P, Plumb R, Pohl CS, Poliakov A, Ponce TC, Ponting CP, Potter S, Quail M, Reymond A, Roe BA, Roskin KM, Rubin EM, Rust AG, Santos R, Sapojnikov V, Schultz B, Schultz J, Schwartz MS, Schwartz S, Scott C, Seaman S, Searle S, Sharpe T, Sheridan A, Shownkeen R, Sims S, Singer JB, Slater G, Smit A, Smith DR, Spencer B, Stabenau A, Stange-Thomann N, Sugnet C, Suyama M, Tesler G, Thompson J, Torrents D, Trevaskis E, Tromp J, Ucla C, Ureta-Vidal A, Vinson JP, Von Niederhausern AC, Wade CM, Wall M, Weber RJ, Weiss RB, Wendl MC, West AP, Wetterstrand K, Wheeler R, Whelan S, Wierzbowski J, Willey D, Williams S, Wilson RK, Winter E, Worley KC, Wyman D, Yang S, Yang SP, Zdobnov EM, Zody MC, Lander ES (2002) Initial sequencing and comparative analysis of the mouse genome. Nature 420: 520-562.

Wells CM, Beenken KE, Smeltzer MS, Courtney HS, Jennings JA, Haggard WO (2018) Ciprofloxacin and rifampin dual antibiotic-loaded biopolymer chitosan sponge for bacterial inhibition. Mil Med 183: 433-444.

Wilde AD, Snyder DJ, Putnam NE, Valentino MD, Hammer ND, Lonergan ZR, Hinger SA, Aysanoa 
EE, Blanchard C, Dunman PM, Wasserman GA, Chen J, Shopsin B, Gilmore MS, Skaar EP, Cassat JE (2015) Bacterial hypoxic responses revealed as critical determinants of the host-pathogen outcome by TnSeq analysis of Staphylococcus aureus invasive infection. PLoS Pathog 11: e1005341. DOI: 10.1371/ journal.ppat.1005341.

Windolf CD, Lögters T, Scholz M, Windolf J, Flohé S (2014) Lysostaphin-coated titan-implants preventing localized osteitis by Staphylococcus aureus in a mouse model. PLoS One 9: e115940. DOI: 10.1371/ journal.pone.0115940.

Windolf CD, Meng W, Lögters TT, MacKenzie CR, Windolf J, Flohé S (2013) Implant-associated localized osteitis in murine femur fracture by biofilm forming Staphylococcus aureus: a novel experimental model. J Orthop Res 31: 2013-2020.

Wu T, Weng Z, Xu J, Wen G, Yu Y, Chai Y (2018) Baicalin alleviates osteomyelitis by regulating TLR2 in the murine model. Pathog Dis 76. DOI: 10.1093/ femspd/ftx123.

Xiao L, Li T, Ding M, Yang J, Rodríguez-Corrales J, LaConte SM, Nacey N, Weiss DB, Jin L, Dorn HC, Li X (2017) Detecting chronic post-traumatic osteomyelitis of mouse tibia via an IL-13R $\alpha 2$ targeted metallofullerene magnetic resonance imaging probe. Bioconjug Chem 28: 649-658.

Yamada KJ, Heim CE, Xi X, Attri KS, Wang D, Zhang W, Singh PK, Bronich TK, Kielian T (2020) Monocyte metabolic reprogramming promotes pro-inflammatory activity and Staphylococcus aureus biofilm clearance. PLoS Pathog 16: e1008354. DOI: 10.1371/journal.ppat.1008354.

Yang D, Wijenayaka AR, Solomon LB, Pederson SM, Findlay DM, Kidd SP, Atkins GJ (2018) Novel insights into Staphylococcus aureus deep bone infections: the involvement of osteocytes. mBio 9: e00415-00418. DOI: 10.1128/mBio.00415-18.

Yokogawa N, Ishikawa M, Nishitani K, Beck CA, Tsuchiya H, Mesfin A, Kates SL, Daiss JL, Xie C, Schwarz EM (2018) Immunotherapy synergizes with debridement and antibiotic therapy in a murine 1-stage exchange model of MRSA implant-associated osteomyelitis: J Orthop Res 36: 1590-1598.

Yoon KS, Fitzgerald RH, Sud S, Song Z, Wooley $\mathrm{PH}$ (1999) Experimental acute hematogenous osteomyelitis in mice. II. Influence of Staphylococcus aureus infection on T-cell immunity. J Orthop Res 17: 382-391.

Yoshii T (2002a) Inhibitory effect of roxithromycin on the local levels of bone-resorbing cytokines in an experimental model of murine osteomyelitis. J Antimicrob Chemother 50: 289-292.

Yoshii T, Magara S, Miyai D, Nishimura H, Kuroki E, Furudoi S, Komori T, Ohbayashi C (2002b) Local levels of interleukin-1beta, $-4,-6$, and tumor necrosis factor alpha in an experimental model of murine osteomyelitis due to Staphylococcus aureus. Cytokine 19: 59-65.

Young SW, Roberts T, Johnson S, Dalton JP, Coleman B, Wiles S (2015) Regional intraosseous administration of prophylactic antibiotics is more effective than systemic administration in a mouse model of TKA. Clin Orthop Relat Res 473: 3573-3584.

Zhang B, Braun BM, Skelly JD, Ayers DC, Song J (2019a) Significant suppression of Staphylococcus aureus colonization on intramedullary Ti6Al4V implants surface-grafted with vancomycin-bearing polymer brushes. ACS Appl Mater Interfaces 11: 28641-28647.

Zhang B, Skelly JD, Braun BM, Ayers DC, Song J (2020) Surface-grafted zwitterionic polymers improve the efficacy of a single antibiotic injection in suppressing Staphylococcus aureus periprosthetic infections. ACS Appl Bio Mater 3: 5896-5904.

Zhang H, Chen C, He J, Zhang J, Liu Z, Sun T (2019b) Anti-PD-1 antibody administration following hip fracture surgery reverses immune dysfunction and decreases susceptibility to infection. Mediators Inflamm 2019: 8492090. DOI: 10.1155/2019/8492090.

Zhu C, Wang J, Cheng T, Li Q, Shen H, Qin H, Cheng M, Zhang X (2014) The potential role of increasing the release of mouse $\beta$ - defensin-14 in the treatment of osteomyelitis in mice: a primary study. PLoS One 9: e86874. DOI: 10.1371/journal. pone.0086874.

Zhu X, Zhang K, Lu K, Shi T, Shen S, Chen X, Dong J, Gong W, Bao Z, Shi Y, Ma Y, Teng H, Jiang Q (2019) Inhibition of pyroptosis attenuates Staphylococcus aureus-induced bone injury in traumatic osteomyelitis. Ann Transl Med 7: 170. DOI: 10.21037/atm.2019.03.40.

Zimmerli W, Trampuz A (2011) Implantassociated infection. In Biofilm Infect.. Editors: Bjarnsholt T, Østrup Jensen P, Moser C, Høiby N. pp: 69-90. New York, NY. Springer New York. DOI: 10.1007/978-1-4419-6084-9_5.

\section{Web References}

1. https://www.webofknowledge.com [15.03.2021] 2. https://pubmed.ncbi.nlm.nih.gov/ [15.03.2021]

\section{Discussion with Reviewer}

Reviewer: There is apparently a wide range of mouse models available. Would the field benefit from standardisation and a reduction to relatively fewer models allowing greater consistency? What may be the pros and cons of this approach?

Authors: This is a very good point to discuss. Indeed, in each osteomyelitis section, the infection has been performed differently and by using a different mouse line, age and gender, different bacterial strain, infectious dose and volume or different evaluation methods. All these variables need to be taken into consideration when the studies are compared. Standardisation could be applied both for in vitro and in vivo assays and would increase the reproducibility of studies between laboratories. Moreover, the 
standardisation of the model and the evaluation methods could be used as a preclinical step to follow to evaluate the efficacy of antimicrobial preventive or treatment strategies (Coenye et al., 2018). However, since there simply is a vast number of relevant clinical parameters that vary from study to study, in many cases, models will need to be tailored to the specific clinical situation. Then, to assess comparability of the models and study outcomes, minimum information guidelines are advised.

Editor's note: The Scientific Editor responsible for this paper was Fintan Moriarty. 